\title{
Pandemic Pedagogy: A Zoom Teaching Experiment Using Cognitive Theory of Multimedia Learning Principles of Multimedia Design
}

\author{
Kevin C. Knoster \\ kcknoster@mix.wvu.edu
}

Follow this and additional works at: https://researchrepository.wvu.edu/etd

Part of the Curriculum and Instruction Commons, Educational Methods Commons, Educational Technology Commons, Higher Education and Teaching Commons, and the Instructional Media Design Commons

\section{Recommended Citation \\ Knoster, Kevin C., "Pandemic Pedagogy: A Zoom Teaching Experiment Using Cognitive Theory of Multimedia Learning Principles of Multimedia Design" (2021). Graduate Theses, Dissertations, and Problem Reports. 8322.}

https://researchrepository.wvu.edu/etd/8322

This Dissertation is protected by copyright and/or related rights. It has been brought to you by the The Research Repository @ WVU with permission from the rights-holder(s). You are free to use this Dissertation in any way that is permitted by the copyright and related rights legislation that applies to your use. For other uses you must obtain permission from the rights-holder(s) directly, unless additional rights are indicated by a Creative Commons license in the record and/ or on the work itself. This Dissertation has been accepted for inclusion in WVU Graduate Theses, Dissertations, and Problem Reports collection by an authorized administrator of The Research Repository @ WVU. For more information, please contact researchrepository@mail.wvu.edu. 
Pandemic Pedagogy:

A Zoom Teaching Experiment Using Cognitive Theory of Multimedia Learning Principles of Multimedia Design

\author{
Kevin C. Knoster
}

Dissertation submitted

to the Eberly College of Arts and Sciences

at West Virginia University

in partial fulfillment of the requirements for the degree of

Doctor of Philosophy in

Communication Studies

\author{
Alan K. Goodboy, Ph.D., Chair \\ Matthew M. Martin, Ph.D. \\ Megan R. Dillow, Ph.D. \\ Zachary D. Johnson, Ph.D.
}

Department of Communication Studies

Morgantown, West Virginia

2021

Keywords: online teaching, Zoom teaching, multimedia instruction, learning, cognitive load, CTML 


\begin{abstract}
Pandemic Pedagogy:

A Zoom Teaching Experiment Using Cognitive Theory of Multimedia Learning Principles of Multimedia Design
\end{abstract}

\title{
Kevin C. Knoster
}

The purpose of this dissertation was to conduct an experimental study exploring the applicability of multimedia principles of effective instructional design to Zoom teaching. In the wake of the COVID-19 pandemic, educators across higher education were forced to rapidly transition from traditional face-to-face instruction to online teaching. One of the most common ways in which colleges and universities navigated this transition in the United States was via mass adoption of the video conferencing platform Zoom. However, best practices have not yet been identified to assist instructors inexperienced with online teaching in adapting to remote instruction via Zoom. This dissertation argued that longstanding principles of effective multimedia designed based on the cognitive theory of multimedia learning (CTML) may be conducive to more effective teaching and enhanced student learning via Zoom - particularly the signaling principle, embodiment principle, and generative activity principle. This dissertation hypothesized that an instructor's implementation of these three multimedia principles in a Zoom lesson would decrease the likelihood of overwhelming students' finite information processing capacity and, in turn, improve students' performance on a post-lesson quiz, as well as inquired whether this effect would vary based upon the extent to which students were self-regulated. This dissertation also hypothesized that an instructor's implementation of these three multimedia principles in a Zoom lesson would increase students' level of reported affect for their instructor. Participants were 140 undergraduate students who were randomly assigned to attend an online lesson in which an instructor either utilized Zoom features to enact CTML-based principles or refrained from doing so. Following the lesson, students completed a questionnaire which included a 10-question test related to the content presented during the lesson, as well as instruments assessing students' working memory overload, familiarity with lesson content, interest in lesson content, GPA, selfregulation, and affect toward their instructor. Findings revealed that that the incorporation of CTML principles during instruction directly improved participants' performance on the postlesson test, as well as increased students' affect toward their instructor. In contrast, participants did not exhibit significantly different levels of working memory overload between experimental conditions, nor did working memory overload mediate the effect of CTML-based instruction on students' post-lesson test scores as hypothesized. Altogether, the results of this dissertation suggest that instructors can enhance their students' online synchronous learning experiences by capitalizing on the affordances of Zoom to enact CTML-based principles of instructional design. These findings, their implications for theory and teaching, and limitations of this dissertation and how they might be addressed by future research are discussed. 


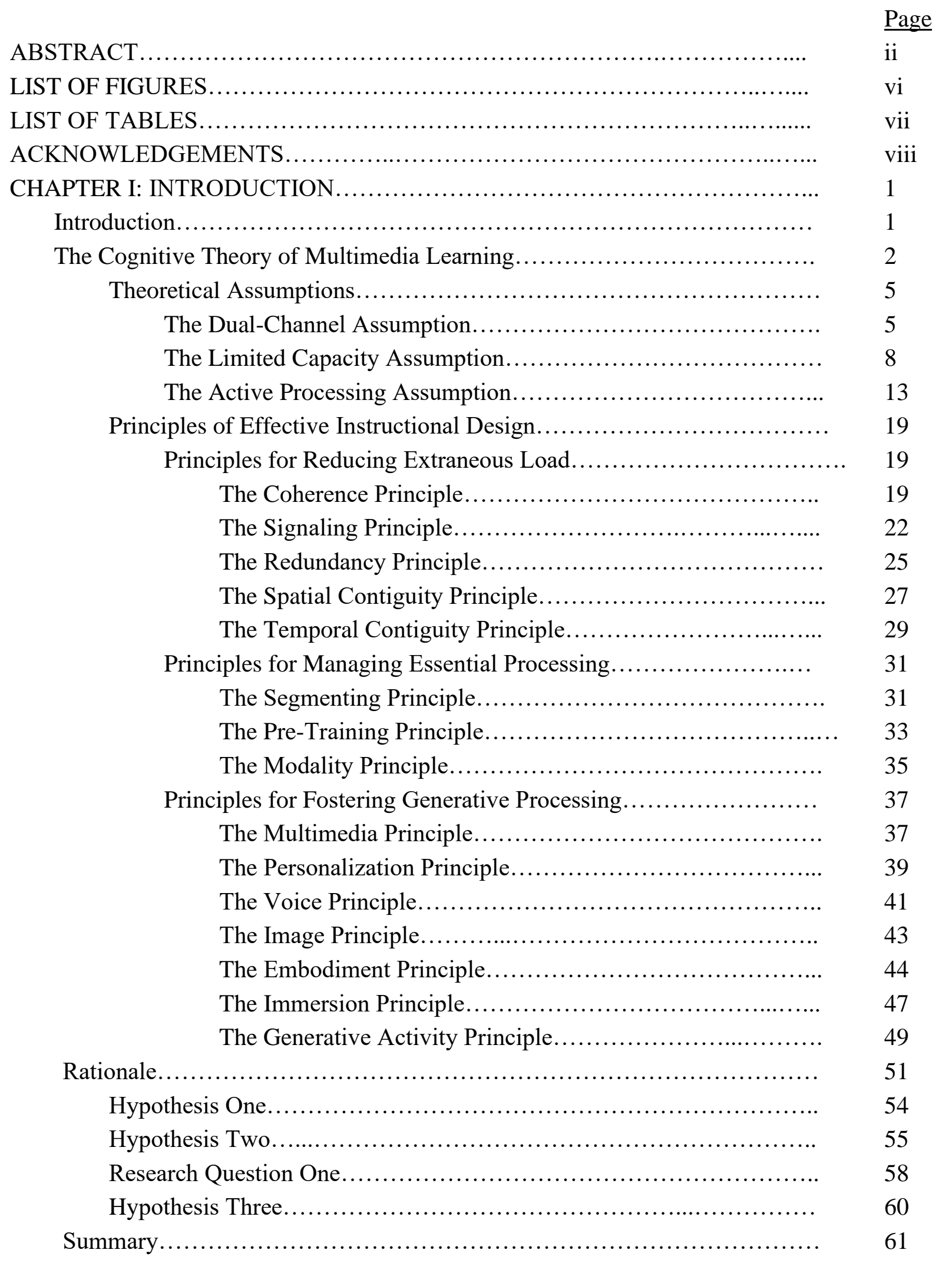




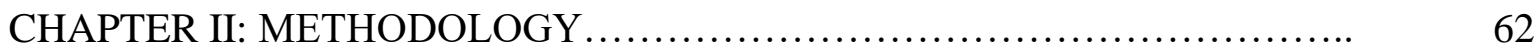

Pilot Study....................................................... $\quad 62$

Participants and Procedures.................................... 62

Instrumentation............................................... 63

Results................................................... 65

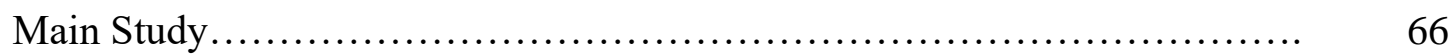

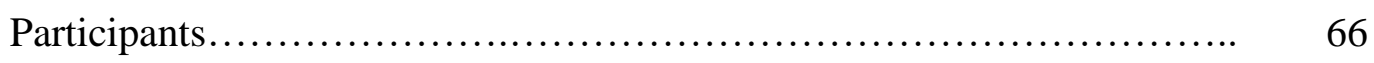

Procedures.......................................................... 67

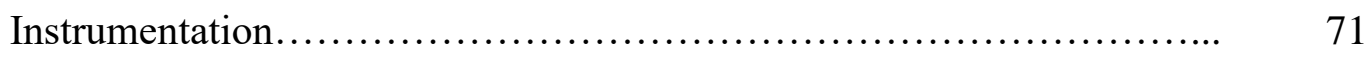

Data Analysis.............................................. 77

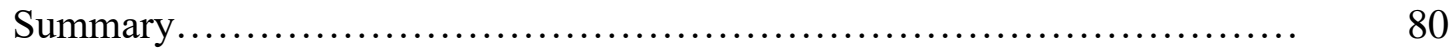

CHAPTER III: RESULTS ........................................... 81

Hypothesis Testing............................................. 81

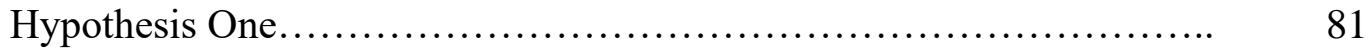

Hypothesis Two............................................ 83

Research Question One........................................ 85

Hypothesis Three............................................. 87

Summary.................................................... 90

CHAPTER IV: DISCUSSION .......................................... 91

CTML Principles, Working Memory Overload, and Learning............... 91

CTML Principles and Student Affect Toward Instructor................... 98

Implications for Theory and Online Teaching............................ 100

Limitations and Future Directions.................................... 106

Summary...................................................... 112

REFERENCES................................................... 113

APPENDICES ...................................................... 155

Appendix A. Pilot Study Lesson Script............................... 155

Appendix B. Pilot Study PowerPoint................................... 168

Appendix C. Pilot Test Survey......................................... 174

Appendix D. Bulletin Board and Online Study Announcement................ 179

Appendix E. Cover Letter............................................ 180

Appendix F. IRB-Approved Email Announcement......................... 182

Appendix G. Online Sign-Up Survey................................... 184

Appendix H. PowerPoint Presentation (Main Experiment)................... 187

Appendix I. Standard Online Lesson Script................................ 193

Appendix J. CTML-based Online Lesson Script.......................... 202 


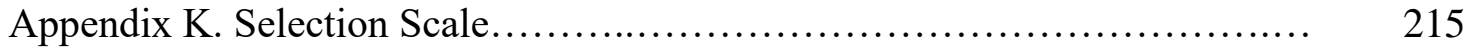

Appendix L. Organization Scale........................................ 216

Appendix M. Working Memory Overload Subscale......................... 217

Appendix N. Post-Lesson Test......................................... 218

Appendix O. Instructional Affect Assessment Instrument.................... 220

Appendix P. Resource Management Subscale.............................. 221

Appendix Q. Perceived Familiarity Subscale............................... 222

Appendix R. GPA Questions............................................. 223

Appendix S. Student Interest Scale...................................... 224 


\section{List of Figures}

$\underline{\text { Page }}$

Figure 1. Hypothesized Conceptual Model of Hypothesis 2..............

Figure 2. Conceptual Model for Research Question $1 \ldots \ldots \ldots \ldots \ldots \ldots . . . . . .$. 
Table 1. Descriptive Information for the 10-Item Test........................ 74

Table 2. Percentages of Correct and Incorrect Answers for the 10-Item Test......... 74

Table 3. Intercorrelations Among Variables................................... 82

Table 4. Unstandardized Model Estimates for Hypothesis $2 \ldots \ldots \ldots \ldots \ldots \ldots \ldots \ldots \ldots . \ldots . \ldots . \ldots$

Table 5. Unstandardized Model Estimates (Including Covariates) for Hypothesis 2.. 85

Table 6. OLS Path Model Coefficients: First Stage Moderated by Self-Regulation... 86

Table 7. OLS Path Model Coefficients: First Stage Moderated by Self-Regulation (Including Covariates) ........................................ 87 


\section{ACKNOWLEDGEMENTS}

It's a surreal feeling sitting down to write this portion of my dissertation. To be honest, I occasionally catch myself needing to double-check that I'm actually awake and this isn't all some dream running through my head as I drool on my desk in Armstrong Hall. My past three years in the Department of Communication Studies at West Virginia University seem like a blur to me as I sit in my apartment and try to reflect on them - thanks in no small part to COVID-19, definitely (to be fair, it did a real number on everybody), but also as a result of the sheer number of incredible opportunities and experiences that I've been blessed with in such a short span of time. More incredible, still, are the amazing people I've been fortunate enough to know during my time here. Whether I already knew them before pursuing my doctorate or met them through the Department of Communication Studies, these people are the real reason that I am sitting where I am and typing this section of my dissertation. Neither this dissertation nor anything else I have done during my time as graduate student has been a solo act. I am where I am because of the wonderful people who have let me stand on their shoulders. And now, at the risk of embarrassing some of them, indulge me while I sing their praises for a little while.

The first person I would like to thank is my wife, Lisa. None of this has any meaning without you, and it would never have happened without you. You are my North star. You are the wind in my sails and my port in the storm. You sacrificed just as much as I did, if not more, to make any of this even remotely possible, and as far as I'm concerned this dissertation belongs to you just as much as it does to me. I know that being with me can't always be easy, and I honestly still have no idea how or why you ultimately decided to shack up with a mouth-breathing knuckle-dragger like me - but every minute I'm with you, you make me a better version of me, and every day I wake up and see you lying next to me is a reminder that I'm the luckiest guy in the whole wide world. You're the most wonderful, warm, amazing, kind, patient, sweet, giving 
person I've ever met, and I'm completely smitten and head-over-heels-to-the-moon-and-backover-the-top-and-out-of-my-mind in love with you.

Next, I'd like to thank my parents, Tim and Marsha. A kid could never have asked for a better mom and dad. Any of the good things that I am come from you (and I promise that the not-so-good things aren't your fault). You are both unconditionally loving and unwaveringly supportive. Mom, you have one of the biggest, most open hearts I've ever seen, and you inspire me to have faith in others and to do what's right even if it's risky or not what's convenient. Dad, you were the one who taught me that "if a job's worth doing then it's worth doing well," that being a good person is worthwhile in-and-of-itself and shouldn't be performative, and you exemplify the kind of man I want to become just by being you. You'll always be Superman to me. If I ever manage to be even half as awesome as either of you are, I'll have turned out okay. Thank you both so much for being you.

To my sisters, Megan and Kelsey, thanks for always having my back - and for always keeping me humble. I know that ragging on each other is sort of our unique, dysfunctional brand of love language, but let me spell it out just in case I don't always say it clearly enough: I love you both so much, and I am so proud of each of you (of who you are and of who you choose to be). I'm incredibly lucky to be your brother, and I look up to you both (metaphorically, not literally since I am much, much taller than either of you).

To my advisor and friend, Dr. Alan Goodboy, thank you for always being in my corner. I've known you since I was a baby Husky back at Bloomsburg University, and it's hard to believe how long or how consistently you've always been there for me. As my mentor and advisor, you were the one who put graduate school on my radar to begin with, and you've continued to provide me with unbelievable opportunities throughout my time at West Virginia 
University - dragging me along kicking and screaming at times, but always for the better in the end (and only occasionally saying "I told you so" after). As my friend, you've been a constant source of guidance and support, and you've stepped up for me and helped me in ways that have made incredible differences in my life and brought me back from the brink on more than a few occasions. I honestly struggle to articulate how much our friendship has meant to me. Thank you for always being there. Our next round at Tin 202 is on me.

To my dissertation committee, Dr. Matthew Martin, Dr. Megan Dillow, and Dr. Zachary Johnson, thank each of you so, so much for all of your time, energy, patience, guidance, and assistance as I cobbled together this dissertation. Dr. Martin, you have been a source of support and mentorship since my time in the M.A. program back in 2013-2014, and you continue to be a role model as far as the type of researcher and educator I want to be. You told me once, in passing, during a meeting in your office that "Whenever I can, if there's a way, I'll always do whatever I can to help a student who needs a hand." I'm not sure if I've ever mentioned it, but that has stuck with me since and it's a mantra I've reflected on whenever I cross paths with a student who might be struggling. Dr. Dillow, thank you for your constant and unflinching support throughout my time in this program. COMM 722 was one of my favorite graduate classes here at WVU, largely just because of you being you, and there have been so many instances when your encouragement and kindness have picked me back up after graduate life knocked me down. Dr. Johnson, it's been awesome to watch the trajectory of your career since you graduated from this program back when I was just starting as an M.A. - it's opened my eyes to what's possible and you're an example of the kind of scholar I'd like to be. Your constant positivity and readiness to always help are incredibly appreciated. Altogether, each of you are frankly amazing and I look at all of you as role models. 
I'd also like to thank the rest of the faculty and staff in the Department of Communication Studies at West Virginia University: Dr. Scott Myers, Dr. Christine Rittenour, Dr. Daniel Totzkay, Dr. Elizabeth Cohen, Joy Green, Terri Kelley, Ryan Thompson, Nikki Loy, Dr. Carrie Kennedy-Lightsey, Dr. Katie Kang, John Cole, and Dr. Brian Patterson. Dr. Myers, Dr. Rittenour, Dr. Totzkay, and Dr. Cohen, I've had the opportunity to learn from each of you at some juncture as either an M.A. or Ph.D. student (and I've hoarded my notes over the years if anyone needs proof), and greatly enjoyed and benefited from those experiences. In that same vein, Dr. Keith Weber and Dr. Nicholas Bowman, although you're no longer at West Virginia University, I also had the chance to learn from each of you during my time as an M.A. student and both of you have continued to be supportive ever since. Last but certainly not least; Joy and Terri, you two make this place run and have been my angels of mercy on more than one occasion - thank you for always being willing to help, and thank you especially for your patience with my printer-illiteracy. Years later, it's a miracle I never broke it (or maybe I did and you just never told me, in which case thank you again).

To Heath Howard, my fellow doctoral cohort member, thank you for your repeated sanity-checks and your patience and empathy in serving as my impromptu counselor and rabbi. You never signed up for the job, but you didn't so much as wince even once. While we saw less of each other once the world went on lockdown thanks to COVID-19, your friendship and support really helped me back on my feet at times when life had put me on my backside. To my fellow Ph.D. students - those who came well before me (e.g., Dr. Sara LaBelle, Dr. Jordan Atkinson, Dr. Dana Borzea, Mary Donato, Dr. Zachary Goldman), slightly before me (e.g., Dr. James Baker, Dr. Cathlin Clark-Gordon, Dr. Evan Watts, Joe Wasserman, Sara Pitts, Dr. Stephen Kromka, Dr. McKay West, Dr. Kylie Wilson), or just after (e.g., Nora Radway, Katie 
Armstrong, Lauren Fellers, Rachael Purtell, Matt Shin, Casey Stratton) - thank each of you for your assistance and support, as well. I would not have made it this far without your help.

Lastly, to my friends outside of this program who have had my back for just about as long as I can remember - there's too many of you to get into the specifics of how you've helped me or what your friendship means to me, but nothing I've accomplished would have been possible without you, too, so you at least deserve some recognition. Robert Grindlinger, Tyler Hott, Brent Swinehart, Amy Calem, Andrew and Alyssa Wagner, Joe and Taylor Stahely, Sean and Erin Bell, Spenser and Caitlin Ercole (finally), Weston and Vanessa Pesillo (Lisa and I are counting the days), Henry Geise, Logan James, Andrew and Emily Gaffney, Scott Schmalzried, Nathan Cybak, Marie Whelan, Avery Sheppard, Yakir Garavelas, Sean Campbell, Ryan Luna, Matthew Lewis, to name only a few - thank you, thank you, thank you all. 


\section{CHAPTER I}

\section{Introduction}

2020 saw tectonic shifts in the educational landscape of the United States. In the wake of the ongoing COVID-19 pandemic, educators across PreK-12 and higher education environments had to unexpectedly transition from face-to-face teaching to online instruction as schools and universities continuously adapted to social distancing mandates and stay-at-home orders (Carrillo \& Flores, 2020; Murphy, 2020; Stafford, 2020). This transition dramatically affected the dynamics of teaching and learning - particularly at colleges and universities where instructors may have lacked sufficient pedagogical content knowledge and experience necessary for effective online teaching (Huber \& Helm, 2020; Kali et al., 2011; Rapanta et al., 2020). Relatedly, data suggest that prospective undergraduate students are reconsidering whether pursuing their degrees at institutions struggling to adapt to online teaching is ultimately worthwhile - questioning both the quality of the online education they will receive and the monetary cost associated with online education (Blagg, 2020; Dworak, 2020; Hubler, 2020). Further, undergraduate students already enrolled at colleges or universities transitioning to online modalities have similarly expressed concerns regarding the extent to which the remote instruction they have received has been conducive to meaningful learning experiences (Hess, 2020; Means \& Neisler, 2020). Given this, there is currently an urgent need to identify evidencebased best online teaching practices to adapt to the unique context and constraints of remote instruction (Carrillo \& Flores, 2020).

One of the most common adaptations colleges and universities have made to pivot to online instruction is through the mass adoption of the video conferencing platform Zoom (Kristóf, 2020; Stafford, 2020). Using Zoom, individuals interact with one another digitally by 
creating and joining an online meeting room with a unique multi-digit identification number.

Users can connect to Zoom meeting rooms using a variety of devices (e.g., desktop computer, laptop computer, mobile phone, tablet), and once they are connected can utilize various program features to communicate; such as high-definition audio and video, screen-share, white-boarding, annotation, breakout rooms, virtual backgrounds, in-meeting chat, local recording, and nonverbal feedback (Zoom, n.d.). The flexibility which Zoom provides its users has made it a popular interface for online instruction - expanding Zoom's daily user base from 10 million users in 2019 to 200 million users in 2020, largely due to its implementation across colleges and universities (Jones, 2020).

However, despite Zoom's comprehensive functionality and relatively intuitive user interface - as well as the litany of positive reviews it has received from educators across PreK-12 and higher education environments - no video-communication software nor program is so robust that it can compensate for a teacher's lack of effective multimedia instructional design. Indeed, while the integration of video and audio has the potential to substantially enhance student learning - particularly in remote instruction - teachers must be deliberate to employ multimedia technologies in a manner which aligns with how their students naturally learn. Given this, the purpose of this dissertation is to explore how longstanding principles of effective multimedia design might be strategically incorporated into online instruction via Zoom in a manner conducive to enhancing students' learning. To achieve this purpose, this dissertation draws upon research grounded in the cognitive theory of multimedia learning (CTML, Mayer, 2001) to identify specific ways in which college and university educators can enhance their online pedagogy using the affordances of Zoom.

The Cognitive Theory of Multimedia Learning (CTML) 
CTML (Mayer, 2001), succinctly, is concerned with how individuals construct knowledge (i.e., learn) from words and pictures. CTML is based on the empirically supported (e.g., Mayer, 1989; Mayer \& Anderson, 1992; Mayer et al., 1996; Mayer \& Gallini, 1990; Moreno \& Mayer, 1999b; Ponce \& Mayer, 2014b; Sung \& Mayer, 2012) premise that individuals generally process information more deeply when words and pictures are presented together than when they are presented in isolation, an idea which Mayer (2001) described as the multimedia principle. However, Mayer (2005a) also cautioned that "simply adding pictures to words does not guarantee an improvement in learning - that is, all multimedia presentations are not equally effective" (p. 31). In fact, presenting words and pictures in certain ways (e.g., simultaneously presenting words and pictures conveying distinct pieces of information, presenting words and pictures in a manner which is not clearly organized) may result in the creation of multimedia messages which ultimately impede learning rather than enhance it undermining students' ability to efficiently process information (e.g., Harp \& Mayer, 1997, 1998; Mayer et al., 2001; Mayer \& Jackson, 2005). One of the foremost goals of CTML is to guide the development of effective multimedia messages based upon consideration of how the human brain functions and the ways in which individuals cognitively process information (Mayer, 2001). That is, the aim of CTML is to design instructional multimedia messages based on the optimal ways that people learn.

CTML's focus on how individuals learn is reflected in Mayer's (2001) conceptualization of a multimedia instructional message (used interchangeably with multimedia instructional presentation and multimedia instruction) as "a presentation involving words and pictures that is intended to foster learning" (p. 3). From a perspective grounded in CTML, learning is an ongoing activity in which individuals engage in knowledge construction; an active process 
whereby learners create mental representations of new instructional material presented to them (Mayer, 2001). Contrary to conceptualizations of learning as a more passive process, such as response strengthening (which assumes that learners receive punishments and rewards that either strengthen or weaken associations between certain stimuli and responses) and information acquisition (which assumes that learners passively add new information to pre-existing memories as they receive it from external sources), Mayer (2001) posited that learners are "active sense makers" (p. 13) who must continuously engage in their own information processing to effectively understand new instructional material and integrate it with previously created knowledge structures. From this perspective, information is not an objective commodity that is transferred uniformly and in its entirety from a source (e.g., a teacher) to a receiver (e.g., a student), but something that is personally created by each individual learner based on the specific ways in which they cognitively process instructional material (as exemplified by different students who, despite being exposed to the same lesson, may nevertheless interpret lesson content in distinct ways from one another; Mayer, 2001).

Thus, the types of information which learners might process, and ultimately the kinds of knowledge they might construct, can vary substantially. Specifically, knowledge may be factual (i.e., knowledge related to terminology and details), conceptual (i.e., knowledge related to categories and classifications, principles, generalizations, theories, models, and structures), procedural (i.e., knowledge related to subject-specific skills, techniques, methods, and criteria for evaluating appropriate procedures), or metacognitive (i.e., strategic knowledge, knowledge about cognitive tasks, and self-knowledge) in nature (Anderson et al., 2001; Mayer, 2011). From the vantage point of CTML, the role of the teacher is to serve as a cognitive guide who facilitates learners' construction of certain types of knowledge by directing learners toward specific 
information they should pay particular attention to, as well as assisting learners in cognitively organizing that information (Mayer, 2001, 2005a, 2014a, 2021). CTML suggests that teachers will be most effective in fulfilling this role when they engage students in active learning, thereby stimulating deep cognitive processing conducive to remembering (i.e., being able to retain, reproduce, or recognize information) and understanding (i.e., being able to construct a coherent mental representation of new information) instructional material (Mayer, 2001, 2005a, 2021).

Remembering and understanding play an important part in shaping students' overall learning outcomes - outcomes which Mayer (2001) characterized as no learning, rote learning, and meaningful learning. According to Mayer (2001), no learning refers to when learners exhibit both poor retention and poor understanding of course topics and concepts. Rote learning occurs when learners exhibit strong retention yet poor understanding; acquiring inert or fragmented knowledge in the form of disparate, isolated pieces of information which cannot be applied in novel contexts (i.e., "factoids"; Mayer, 2001, p. 17). Meaningful learning occurs when learners demonstrate both strong retention and understanding of instructional material, fully integrating new information into previously constructed cognitive schemas and developing the ability to effectively access and apply that knowledge when solving new problems in unique situations (Mayer, 2001). CTML (Mayer, 2001) identifies strategies which instructors can use to assist their students in achieving meaningful learning outcomes, all of which are ultimately grounded in three fundamental theoretical assumptions: (1) the dual-channel assumption, (2) the limited capacity assumption, and (3) the active processing assumption.

\section{Theoretical Assumptions}

\section{The Dual-Channel Assumption}

The dual-channel assumption of CTML is that human beings use different processing 
channels to make sense of information that they see and hear (Mayer, 2001). Specifically, content presented via readable text, animations, pictures, video, or other visual representations are detected through individuals' eyes and processed via the visual information channel, whereas content presented via narration, verbal explanation, or other sounds are detected by the ears and processed via the auditory information channel (Mayer, 2001, 2005a, 2014a, 2021). Mayer (2001) suggested that there are two ways of distinguishing the visual and auditory channels from one another - presentation modes and sensory modalities. Comparing information processing channels based on presentation modes, grounded in Paivio's (1986) dual-coding theory, entails an emphasis on whether the particular stimulus used to present a piece of information is verbal (e.g., written words, spoken words) or nonverbal (e.g., sound effects, animations, illustrations) in nature. In contrast, a sensory modalities approach is primarily concerned with whether a stimulus is detected and interpreted using the eyes (i.e., a learner sees the information) or the ears (i.e., a learner hears the information; Mayer, 2001). CTML, according to Mayer (2001, 2005a), represents a compromise between these two perspectives and adopts both simultaneously employing a sensory modalities approach to differentiate between visual and auditory presentations of information while using a presentation mode approach to differentiate how learners construct pictorially-based cognitive models from models that are verbally-based.

Research exploring the nature of dual-channel information processing has consistently been focused on the implications of a dual-channel system for how individuals remember (i.e., learn) different types of information (Mayer, 2021; Paivio, 2006). Prior to formally articulating a theory of dual coding in 1986, Paivio (1965) found support for the presence of distinct cognitive systems for processing linguistic and nonlinguistic information. Specifically, Paivio (1965) conducted experiments examining imagery as a mediator of different verbal associations, 
reporting that participants were better able to recall concrete words (i.e., nouns referring to tangible objects) than abstract words (i.e., nouns referring to intangible ideas or concepts) due to the comparative ease with which participants were able to construct representative mental images for concrete words. In another study exploring whether pairing images with words facilitated greater recall among young children, Dilley and Paivio (1968) found that the order in which children were presented with paired pictures and words (as well as whether words were paired with other words or whether pictures were paired with other pictures) significantly influenced children's recall. Numerous studies (e.g., Begg, 1972; Richardson, 2003; Sadoski, 1983, 1985; Thompson \& Paivio, 1994) provide similar evidence to support Paivio's (1986) contention that the human brain is comprised of distinct subsystems which specialize in making sense of linguistic and nonlinguistic information, lending credence to the incorporation of presentation modes into CTML's (Mayer, 2001) dual-channel assumption for information processing insofar as it influences learners' construction of visually- or verbally-based mental representations of knowledge.

Ample research also supports Mayer's (2001) incorporation of a sensory modalities approach into CTML's dual-channel assumption. Indeed, numerous longstanding models of human cognition entail an underlying assumption that the human brain makes sense of visual and auditory signals differently, such as Baddely's (1999) model of working memory, Penney's (1989) "separate streams" model, and Sweller's (1988) cognitive load theory (Mayer, 2001, 2005a, 2021). A test of cognitive load theory conducted by Mousavi et al. (1995), for example, was one of the first studies to empirically demonstrate the presence of instructional modality effects (i.e., differences in instructional outcomes attributable to how instructional content is presented visually and auditorily) on students' immediate information processing using working 
memory. Across three experiments in which the materials presented in a geometry lesson were manipulated to be either exclusively visual or a combination of both visual and auditory, students' ability to effectively process information was enhanced when materials were presented using mixed modalities. In another study, Mayer and Moreno (1998) conducted an experiment assessing whether students learned more from a multimedia lesson in which lesson content was presented through both visual (e.g., animation) and auditory (e.g., narration) channels compared to a lesson in which content was presented visually alone (e.g., animation accompanied by written text), reporting that students performed best on transfer tests when they were exposed to visual representations of lesson content accompanied by auditory explanations. The results of this study were replicated in a separate experiment by Moreno and Mayer (1999a). Ginns (2005), in a meta-analysis of 43 different studies exploring the impact of presenting information via visual- and auditory-based channels on student learning, reported findings consistent with the notion that whether students process information visually or auditorily can significantly affect the ways in which they cognitively process, and ultimately retain, that information. In particular, when information was presented using a combination of auditory and visual formats, overall learning was consistently improved. Therefore, when instructors in multimedia environments present words and pictures to students in a manner which considers how the visual and auditory information channels process information, learning can be meaningfully enhanced (Mayer, 1989; Mayer, 2005b, 2021; Mayer \& Anderson, 1992; Mayer et al., 1996; Mayer \& Gallini, 1990; Moreno \& Mayer, 1999b; Ponce \& Mayer, 2014b; Sung \& Mayer, 2012).

\section{The Limited Capacity Assumption}

The limited capacity assumption, heavily influenced by Baddeley's (1999) theory of working memory and Sweller's (1988) cognitive load theory, stipulates there is a cap on the 
amount of information the human brain can process via the visual and auditory channels at a single point in time (Mayer, 2001). Specifically, individuals can only detect, retain, and interpret so many spoken words or other sounds in a given moment, and can similarly only process a handful of visually presented images simultaneously in their transient sensory memory (Mayer, 2001, 2005a). Sensory memory refers to the briefest moments in which sensations such as sight and sound are initially detected by the eyes and ears and signaled to the brain, which holds fleeting "sensory copies of incoming words and pictures" in the form of "iconic and auditory sensory representations" (Mayer, 2021, p. 42). These sensory representations reflect "portions of the presented material rather than an exact copy of the presented material" (Mayer, 2005a, p. 35). Once this occurs, sensory representations which individuals pay particular attention to (deliberately or otherwise) are subsequently selected and processed in the working memory (Baddeley, 1986; Mayer, 2005a).

Working memory is "used for temporally holding and manipulating knowledge in active consciousness" (Mayer, 2021, p. 41) and is the memory store in which sensory inputs are transformed into fully-fledged, concrete pictorial and verbal representations. The number of representations which can be simultaneously stored in the working memory, however, is extremely finite - with most individuals only being able to hold an average of approximately seven "chunks" (i.e., integrated pieces of information wherein remembering one piece assists in remembering the next) of information in their working memory at a time (Baddeley, 1997). Given this constraint, learners must be cognizant of which specific pieces of information they are presented with that they should pay particular attention to, as committing cognitive resources to unnecessary or superfluous information can quickly overwhelm the working memory's finite processing capacity (Mayer, 2001). When learners effectively employ metacognitive strategies 
(i.e., "techniques for allocating, monitoring, coordinating, and adjusting... limited cognitive resources" [Mayer, 2005a, p. 36]), representations arranged within the working memory can be used to construct more organized and meaningful pictorial and verbal models of information (i.e., representations of important information and how they relate to one another) conducive to being transferred to learners' long-term memory. The transfer of information from the working memory to the long-term memory - in which pictorial and visual models are integrated both with one another and with pre-existing knowledge to be stored indefinitely - constitutes the fundamental process whereby learning occurs from the theoretical perspective of CTML (Mayer, 2001, 2005a, 2014a, 2021). Given this, Mayer (2021) wrote that "the central work of multimedia learning takes place in working memory" (p. 41), characterizing working memory as the key mechanism through which information detected by the senses is interpreted, processed, and transferred to long-term memory in the form of cognitive knowledge structures.

One of the primary goals driving CTML is thus to provide guidance concerning the ways in which instructors can design instructional multimedia messages in a manner which does not overwhelm learners' limited working memory capacity (Mayer, 2001, 2005a, 2014a, 2021). CTML (Mayer, 2001), like CLT (Sweller, 1988), is grounded in the assumption that the effectiveness with which the working memory is able to process new information and subsequently transfer it into longer-lasting memory storage is contingent upon the innate complexity of the information to be learned and how that information is presented. Regarding the former, both the number of informational elements (i.e., "anything that needs to be learned or processed, or has been learned or processed" [p. 58]) contained in an instructional message and the interactivity of those elements (i.e., the extent to which informational elements are logically related to one another) inherently influence the cognitive demands placed upon working 
memory, such that the working memory must process interactive elements simultaneously to make sense of information (Sweller et al., 2011). Element interactivity thus has significant implications for learners' cognitive processing, with highly complex information entailing greater element interactivity placing more cognitive burden on the finite processing capacity of the working memory (Sweller, 1994; Sweller et al., 2011). Beyond innate complexity and element interactivity, the specific ways in which information is presented to learners can also affect the amount of effort required from the working memory to effectively process that information. Characteristics of an instructional message or learning task such as format, modality, time constraints, and pacing (Paas et al., 2003) each contribute to the processing demands placed upon working memory over and above the nature of the information to be processed in and of itself. The inherent complexity of information and the specific manner in which information is presented to learners can thus quickly overwhelm the finite processing capacity of the working memory through the imposition of cognitive load (Sweller, 1988; Sweller et al., 2011).

Cognitive load refers to "a multidimensional construct representing the load that performing a particular task imposes on the learner's cognitive system" (Paas \& van Merriënboer, 1994). The greater the cognitive load associated with a given instructional message or learning task, the greater the burden placed upon the working memory to process it (Sweller et al., 2011). CLT (Sweller, 1988) and CTML (Mayer, 2001) both delineate three distinct types of cognitive load: intrinsic load, extraneous load, and germane load. The amount of intrinsic load presented by novel information is based upon the innate nature of the processes necessary for the information in question to be learned (Sweller, 1994; Sweller \& Chandler, 1994). Put simply, intrinsic load represents the inherent difficulty of a learning task irrespective of how the learning task is presented (Sweller et al., 2011). Contrary to intrinsic load, extraneous load is grounded 
upon instructional procedures and design (Sweller, 2010). Extraneous load emerges due to the specific ways in which instructional messages and learning tasks are presented, such that extraneous load increases when learners are required to put greater effort into accomplishing a learning task based upon the manner in which they are exposed to it (Sweller, 1994). Examples of this include scenarios in which learners are required to seek out additional information to make sense of a particular learning task at hand, must engage in unnecessary or tedious problemsolving processes without receiving practical guidance, or need to reorganize previously acquired learning materials and resources which were provided to them in a disorganized manner (Sweller et al., 2011). Succinctly, extraneous load arises when learning tasks are presented inefficiently (Sweller, 2010).

The intrinsic and extraneous load imposed on the working memory by instructional messages and learning tasks must each be addressed through the allocation of finite cognitive resources. CLT (Sweller, 1988) and CTML (Mayer, 2005a) each characterize the cognitive resources committed to processing intrinsic load as germane resources, given that demands placed upon the working memory due to the inherent nature of information to be processed is fundamentally necessary for learning that information (i.e., the demands cannot be avoided for learning to occur). Research consistently refers to these germane resources as germane load, conceptualized as a unique form of cognitive load devoted to "information that is relevant or germane to learning" (Sweller et al., 2011, p. 57). In contrast, cognitive resources committed to processing the extraneous load imposed by a particular instructional message or learning task are not innately germane to the learning process. These cognitive resources are described as extraneous resources in that they inefficiently expend the finite processing power of the working memory to make sense of superfluous, redundant, or otherwise unnecessary informational 
elements stemming from poorly conceived or implemented instructional design (Sweller et al., 2011).

Despite these differences, intrinsic and extraneous load are additively related to one another insofar as they determine the total overall amount of cognitive load placed upon the finite processing capacity of the working memory. When the working memory's limited cognitive resources are overwhelmed by the demands imposed by intrinsic and extraneous load, it will not be able to process the necessary information for learning to occur. If instructional messages or learning tasks are presented in ways which impose high extraneous load, learners' working memory will commit extraneous resources at the expense of germane resources conducive to effectively processing intrinsic load - ultimately resulting in insufficient germane resources to address the intrinsic load of a given learning task (Sweller, 2010; Sweller et al., 2011). Thus, CTML (Mayer, 2001) is intended to serve as a theoretical framework for designing multimedia instructional messages in a manner which does not overwhelm learners' limited working memory capacity in the form of extraneous cognitive load, instead assisting learning via the provision of cognitive resources germane to it (Mayer, 2005a).

\section{The Active Processing Assumption}

The active processing assumption, the third assumption of CTML, is that human beings must actively engage in cognitive processing if they are to successfully construct coherent and meaningful mental representations of information (Mayer, 2001). In particular, CTML assumes that achieving meaningful learning outcomes is contingent upon individuals selecting, organizing, and integrating the information they are presented with appropriately (Mayer, 2001, 2005a). The process of selection entails recognizing and paying attention to relevant information during instruction, facilitating its transfer from sensory memory to working memory. Organizing 
refers to the arrangement of the information which learners select into concrete pictorial or verbal mental representations within the working memory. Integrating occurs when learners establish connections between recently constructed mental representations and link them to preexisting knowledge structures, anchoring new information with content already stored in the long-term memory (Mayer, 2001, 2005a, 2014a; Wittrock, 1989). CTML thus characterizes the process of learning as highly deliberate and cognitively intensive rather than passive, conceptualizing humans as "active processors who seek to make sense of multimedia presentations" (Mayer, 2005a, p. 36).

Working from this assumption, Mayer (2001) identified five specific cognitive processes entailing selection, organization, and integration which are necessary for learners to achieve meaningful learning outcomes in multimedia environments: (1) selecting relevant (i.e., related to a given learning task) words to be cognitively processed in one's verbal working memory, (2) selecting relevant images to be cognitively processed in one's visual working memory, (3) organizing selected words into cohesive verbal models, (4) organizing selected images into cohesive pictorial models, and (5) integrating verbal and pictorial models with one another and with prior knowledge already stored in the long-term memory. Incorporating the first assumption of CTML (that human beings use different cognitive processing channels to make sense of information that they see and hear), selecting relevant words "involves a change in knowledge representation from the external presentation of spoken words... to a sensory representation of sounds, to an internal working memory representation of word sounds" (Mayer, 2005a, p. 38). Elaborating further, Mayer (2005a) characterized verbal messages as an input and sounds as an output, with learners' active selection of relevant words mediating the conversion of verbal messages to sounds. Specifically, when words are presented to learners via speech, they are 
detected by the ears and processed through the auditory sensory memory. When words are presented to learners as readable text, they are detected by the eyes and processed through the visual sensory memory - later moving to the auditory channel as learners mentally articulate selected printed words (Mayer, 2001, 2005a). Given the second assumption of CTML (that there is a cap on the amount of information that the human brain can process via the visual and auditory channels at a single point in time), learners must select only the most important pieces of the verbal messages they are presented with in order to refrain from overloading their limited cognitive processing capacity. Therefore, learners need to determine "which words are most relevant - an activity that is consistent with the view of the learner as an active sense maker" (Mayer, 2005a, p. 39).

Similarly, the process of selecting relevant images involves "a change in knowledge representation from the external presentation of pictures... to a sensory representation of unanalyzed visual images, to an internal representation in working memory" (Mayer, 2005a, p. 39). In this process, Mayer (2001, 2005a) identified graphical portions of multimedia messages as inputs and mental representations of those graphical portions in the form of mental images as outputs, with the process of selecting relevant images mediating the conversion of graphical portions of multimedia messages into mental images. In contrast to the selection of relevant words, which takes place primarily through the auditory channel, the selection of relevant images takes place primarily through the visual channel. Given the finite processing power of the human brain, learners must select only part of the graphical multimedia messages they are presented with in order to avoid overloading their cognitive systems. Thus, learners need to determine which images are most relevant for interpreting the multimedia presentations they are presented with, necessitating active engagement with and consideration of those presentations (Mayer, 
2005a).

The transformation of spoken words into mental representations of sounds and pictorial portions of multimedia messages into mental images within the working memory sets the stage for the organization of those representations into verbal models (i.e., coherent knowledge structures which organize words) and pictorial models (i.e., coherent knowledge structures which organize images; Mayer, 2001, 2005a, 2021). As verbal models are organized via the auditory processing channel and pictorial models are organized via the visual processing channel, learners must continue to be selective given the limited information processing capacity of the working memory. In particular, learners must prioritize expending their finite cognitive resources on organizing only the most relevant knowledge structures when creating verbal and pictorial models rather than attempting to build as many connections as possible, requiring learners' continued active involvement and decision-making in their own cognitive processing (Mayer, 2001, 2005a).

The integration of word-based and image-based representations in the working memory with one another and previously-constructed knowledge already stored indefinitely in the longterm memory constitutes what Mayer (2005a) described as "perhaps the most crucial step in multimedia learning" (p. 40). This integration occurs when learners establish connections between novel information and prior knowledge, necessitating coordination between the working memory and long-term memory to facilitate the transfer of short-lived, relatively fleeting verbal and pictorial models into enduring cognitive structures. This transfer "reflects the epitome of sense-making because the learner must focus on the underlying structure of the visual and verbal representations" (Mayer, 2005a, p. 40), using previously learned information to solidify new mental representations and knowledge structures. 
The distinct mental representations and knowledge structures which learners construct throughout these cognitive processes, which Mayer (2001, 2005a) conceptualized as mental models, may vary in scope and purpose. Based on the work of Cook and Meyer (1988), Mayer (2001, 2005a) identified five specific types of basic knowledge structures which a person may use to represent and make sense of information they are presented with: process structures, comparison structures, enumeration structures, classification structures, and generalization structures. Mayer (2001) wrote that process structures causally link information, connecting otherwise disparate pieces of information insofar as one influences some change in the other (e.g., explaining how exhaust from automobiles can cause environmental changes). Comparison structures differentiate information, sometimes contrasting multiple elements of that information across several different dimensions (e.g., juxtaposing two different theoretical explanations for a specific phenomenon). Enumeration structures are comprised of lists or collections of subordinate pieces of information related to a broader, overarching topic (e.g., identifying different types of trees). Classification structures, somewhat similarly, are mental representations of hierarchies in which information is organized into subsets or tiers (e.g., outlining the different taxonomic ranks used for biological classification and identifying different subgroups for each rank). Lastly, generalization structures refer to mental representations of information and knowledge which identify a series of main or overall ideas related to a particular topic, each of which are associated with an array of more specific supporting details (e.g., writing an essay on an author's use of themes in a novel and supporting one's position with details from the novel; Mayer, 2001). Based upon the types of knowledge structures that learners may create based off the information they are presented with, Mayer (2021) identified two key implications for multimedia instructional design: (1) that instructional content should conform to a coherent 
structure and (2) that instructional messages should guide learners in developing knowledge structures. In effect, "multimedia design can be conceptualized as an attempt to assist learners in their model-building efforts" (Mayer, 2021, p. 39).

Incorporating CLT's (Sweller, 1988) conceptualization of cognitive load and its implications for information processing, CTML (Mayer, 2001) suggests that each of the five cognitive processes (selecting relevant words, selecting relevant images, organizing words, organizing images, and integrating) place unique demands on learners' cognitive systems. As learners select relevant words and images, they engage in essential processing - or, "processing required to represent the essential material in working memory... caused by the complexity of the material" (Mayer, 2021, p. 51). Essential cognitive processing thus constitutes the amount of mental work that is fundamentally necessary to mentally represent instructional content, driven largely by the number of informational elements, and interactivity of those elements, comprising that content (Mayer, 2005a). In other words, essential processing refers to cognitive processing which addresses intrinsic load. As learners organize selected words and images into verbal and pictorial models and integrate those models with prior knowledge, they engage in generative processing - or, "processing aimed at making sense of the presented material" (Mayer, 2021, p. 52). Generative processing is effectively synonymous with the concept of germane load in CLT (Mayer, 2021; Paas \& Sweller, 2014; Sweller et al., 2011) insofar as it describes the cognitive construction of integrated mental models and is largely contingent upon learners' motivation to understand material (Mayer, 2021). In contrast to essential and generative processing, extraneous processing is conducive to neither selecting, organizing, nor integrating novel information, and is characterized as "processing that does not serve the instructional goal" (Mayer, 2021, p. 51). As reviewed previously, extraneous processing (i.e., extraneous load) arises in scenarios where 
instructors implement poor instructional design, and quickly consumes learners' finite cognitive capacity for making sense of novel information. When this occurs, there is insufficient cognitive capacity remaining to engage in either essential or generative processing, thus inhibiting learning (Mayer, 2001, 2005a, 2014a, 2021).

Given this, Mayer $(2001,2005 a)$ emphasized the importance of instructors in multimedia environments being strategic in their implementation of effective instructional designs. Effective instructional designs in multimedia environments are any designs which enable instructors to achieve three key goals: (1) assist learners in reducing their extraneous cognitive processing, (2) support learners in managing their essential cognitive processing, and (3) help facilitate learners' generative cognitive processing (Mayer, 2001, 2005a, 2021). To that end, Mayer (2021)

reviewed extant research on best instructional practices in multimedia contexts and identified a series of 15 instructional principles conducive to achieving those goals, reviewing each in detail specifically, the (1) coherence, (2) signaling, (3) redundancy, (4) spatial contiguity, and (5) temporal contiguity principles for reducing extraneous processing; the (6) segmenting, (7) pretraining, and (8) modality principles for managing essential processing; and the (9) multimedia, (10) personalization, (11) voice, (12) image, (13) embodiment, (14) immersion, and (15) generative activity principles for fostering generative processing.

\section{Principles of Effective Instructional Design}

Principles for Reducing Extraneous Processing

The Coherence Principle. Perhaps the most straightforward way in which instructors can assist learners in reducing their extraneous cognitive processing is by eliminating extraneous material from their lessons. Mayer (2001) referred to this instructional practice as the coherence principle, writing that "people better understand an explanation from a multimedia lesson 
containing essential material (concise lesson) than from a multimedia lesson containing essential material and additional material (expanded lesson)" (Mayer, 2021, p. 144). Succinctly, the coherence principle suggests that instructors should take steps to ensure that their lessons are no longer than absolutely necessary. While this notion may seem intuitive at first, research suggests that there are numerous reasons why instructors might actually think it is advantageous to supplement their lessons with additional material - particularly when instructors seek to enhance the extent to which students find a lesson interesting through the incorporation of seductive details (i.e., interesting but irrelevant material; Garner et al., 1989) and seductive illustrations (i.e., interesting but irrelevant illustrations; Harp \& Mayer, 1997, 1998). Unfortunately, unnecessary supplemental materials - no matter how seductive - may ultimately undermine learning more than they enhance it, distracting learners' attention away from relevant information, disrupting learner's creation of coherent mental representations of instructional content, and impeding learners' integration of novel information with prior knowledge (Mayer, 2021). The theoretical basis for the coherence principle, ultimately, is that it is best to nip it in the bud when it comes to extraneous processing. That is, given that extraneous processing competes for cognitive resources in the finite working memory and ultimately inhibits essential and generative processing from occurring, it is best instructional practice to remove any unnecessary material from a lesson which may arouse extraneous processing in the first place (Mayer, 2001, 2005c).

Mayer (2021) catalogued numerous studies providing empirical support for the coherence principle. Harp and Mayer (1997, 1998) and Mayer et al. (2001), for example, conducted a series of experiments in which undergraduate students participated in multimedia lessons centered on lightning formations and patterns. Participants were provided with either a concise booklet of 
lesson materials (comprised of five paragraphs of essential information and five corresponding illustrations) or an expanded booklet of lesson materials (comprised of the same five paragraphs and five corresponding illustrations accompanied by compelling captions and stories involving lighting), performing better on post-lesson transfer tests when provided with the concise booklet than the expanded booklet. Mayer et al. (2008) conducted similar experiments exploring the applicability of the coherence principle to computer-based multimedia lessons, reporting that the inclusion of seductive details diminished participant performance on post-lesson assessments. Interestingly, the negative influence of seductive details on assessment performance was moderated by how interested participants became with the seductive details included in lessons, such that participants' understanding of lesson content decreased as the interestingness of seductive details increased - corroborating prior research suggesting that seductive details may distract learners' attention from relevant information and, as a result, learners remember seductive details better than information aligned with instructional goals (e.g., Garner et al., 1991; Hidi \& Baird, 1986). Sung and Mayer (2012) conducted an experiment exploring differences in learning outcomes based upon whether multimedia lessons included instructive graphics, seductive graphics, decorative graphics, or no graphics, finding that graphics which were relevant to instructional goals ultimately contributed most to improvements in participants' learning, whereas participants exposed to seductive graphics performed even worse on postlesson assessments of learning than participants exposed to no graphics at all. Given this, Sung and Mayer (2012) ultimately concluded that "learners are more likely to engage in instructionally appropriate cognitive processing when they receive instructive graphics which draw their attention toward the essential content than when they receive seductive graphics which draw their attention away from the essential content" (p. 1623). These studies, among others (e.g., 
Garner et al., 1989; Hidi \& Baird, 1988; Korbach et al., 2016; Rey, 2016; Sanchez \& Wiley, 2006; Wang \& Adesope, 2017; Wang et al., 2017; Yue \& Bjork, 2017), lend ample empirical support to CTML's coherence principle of effective instructional design, suggesting that one of the most effective ways in which instructors can reduce learners' extraneous processing is by refraining from introducing extraneous content.

Similarly, in instructional communication, coherence constitutes one of five dimensions comprising Bolkan's (2017a) Clarity Indicators Scale (CIS) - an instrument developed to measure instructor clarity. Clarity refers to a student's "perception that various low- and intermediate-inference behaviors, enacted by a teacher, assist students in selecting, understanding, and remembering the structure and details of information" (Titsworth \& Mazer, 2016, p. 112). Bolkan (2017a) argued that coherence is a dimension of instructor clarity because "when teachers provide extra information, they force students to spend cognitive resources determining what to focus on" (p. 22). Regardless of how interesting extra information (i.e., seductive details) may be to learners, its incorporation into a lesson nevertheless impedes learners' ability to effectively select, organize, and integrate information that is relevant to overarching instructional goals given the finite cognitive capacity available to process novel information via working memory (Mayer, 2001, 2005c, 2014a, 2021). Given this, from an instructional communication perspective, the coherence principle of CTML constitutes a conceptual cornerstone of instructional clarity.

The Signaling Principle. The signaling principle suggests that instructors can assist learners in reducing their extraneous processing by employing effective organizational structure in their lessons - or, that "people learn more deeply from a multimedia message when cues are added that highlight the organization of essential material" (Mayer, 2005c, p. 184). In scenarios 
where extraneous material cannot be outright removed from lessons, the signaling principle suggests a viable solution is to integrate cues within those lessons which draw learners' attention away from extraneous details and toward essential material (Mayer, 2021). Instructors can apply the signaling principle in various ways while teaching in multimedia environments, such as through verbal signaling (i.e., directing learners' attention to specific verbal information) or visual signaling (i.e., directing learners' attention to specific pictures or graphics). Examples of verbal signaling include classic signaling (i.e., directing learners' attention to specific information through the use of traditional organizational cues such as outlines, headings, and pointer words; Harp \& Mayer, 1998; Loman \& Mayer, 1983; Mautone \& Mayer, 2001; Mayer et al., 1984), spatial outlines (i.e., visual arrangements of important words into organizational structures such as matrices, charts, or hierarchies; Ponce \& Mayer, 2014a, 2014b; Ponce et al., 2018; Stull \& Mayer, 2007), and highlighting (i.e., manipulating the font or color of particular words or emphasizing particular words when spoken aloud; Mayer, 2021; Ponce \& Mayer, 2014b; Ponce et al., 2018). Examples of visual signaling include instructors' use of distinctive colors to emphasize particular parts of a graphic (Mautone \& Mayer, 2001; Wang et al., 2018; Xie et al., 2019), coordinating visual and verbal cues so that graphical changes coincide with the presentation of verbal information (Xie et al., 2019), and specific cues such as pointing gestures, arrows, flashing, and graying out text (Li et al., 2019; Mayer, 2021; Wang et al., 2018). While signals do not add any new information to lessons, they add organization and structure to guide learners' attention (Mayer, 2005c). Through verbal and visual signaling, instructors may serve as guides for learners' cognitive processing by drawing attention to important instructional material and assisting learners in selecting relevant information and organizing that information into coherent mental representations. "Without guidance in how to carry out appropriate cognitive 
processing, the learner is more likely to engage in extraneous cognitive processing - such as processing extraneous material and trying to organize it with the rest of the material" (Mayer, 2021, p. 171).

Research has provided consistent empirical support for specific aspects of CTML's signaling principle (Mayer, 2005c, 2014a, 2021); in particular, that instructors' use of classic signaling (Harp \& Mayer, 1998; Loman \& Mayer, 1983; Mautone \& Mayer, 2001; Mayer et al., 1984), spatial outlines (Ponce \& Mayer, 2014a, 2014b; Ponce et al., 2018; Stull \& Mayer, 2007), color coding (Mautone \& Mayer, 2001; Wang et al., 2018; Xie et al., 2019), and specific (as opposed to general) pointing gestures (Li et al., 2019) assist learners in reducing extraneous processing and lead to improvements in learning. In contrast, evidence is mixed concerning the effectiveness of highlighting (Ponce \& Mayer, 2014b; Ponce et al., 2018; Xie et al., 2019) in instructional design, and it has been suggested that too many signals from instructors may prove counterproductive and overwhelm learners' cognitive processing (Stull \& Mayer, 2007). Studies further suggest that different signaling techniques might prove more or less effective for assisting certain types of learners in reducing extraneous processing under specific conditions, varying based on learners' reading ability (Mayer et al., 1980; Naumann et al., 2007) and informational complexity (Jeung et al., 1997). Nevertheless, meta-analyses (e.g., Richter et al., 2016; Schneider et al., 2018) lend general support to CTML's signaling principle, leading Mayer (2021) to argue that instructors in multimedia environments should make conscious efforts to incorporate signaling into their teaching - characterizing signaling as a highly effective instructional technique for reducing learners' extraneous processing in the presence of extraneous material.

Signaling has also been examined from an instructional communication perspective (e.g., Bolkan, 2017a, 2017b; Bolkan et al., 2016, 2017; Titsworth \& Kiewra, 2004). Signaling, like 
coherence, is one of five dimensions comprising the CIS developed by Bolkan (2017a) theoretically tethering the signaling principle (Mayer, 2005c) with instructional communication research concerned with the construct of instructor clarity. Moreover, both the Ohio State studies (e.g., Bush et al., 1977; Kennedy et al., 1978) and Land and Smith studies (e.g., Land, 1979, 1981) examined the organization of instructional material as a key component of clear teaching, and ample instructional communication scholarship has scrutinized the ways in which instructors' use of cues (e.g., Titsworth, 2001, 2004; Titsworth \& Kiewra, 2004) and manipulation of organizational structure (e.g., Chesebro, 2003) can enhance students' perceptions of clarity and, subsequently, understanding of lesson content. Instructional communication research thus generally affirms literature in educational psychology suggesting that effective signaling through instructional cues and organization can assist students in learning - facilitating the selection of relevant information and the organization of that information into coherent mental representations (e.g., Bolkan, 2016, 2017b; Bolkan et al., 2016; Bolkan et al., 2017; Titsworth et al., 2015; Titsworth \& Mazer, 2016; Mazer, 2018).

The Redundancy Principle. The redundancy principle of CTML (Mayer, 2001) suggests that learners do not understand material better when printed text is presented alongside graphics and narration - rather, learning is enhanced by removing printed text in scenarios where graphical representations of instructional content are already accompanied by narration, particularly when instruction is fast-paced. That is, "another way to reduce extraneous cognitive load... is to refrain from adding redundant on-screen text" (Mayer, 2005c, p. 192). When information presented through the visual channel is identical to information presented through the auditory channel, that information becomes redundant. Redundancy facilitates extraneous processing as the visual channel becomes overwhelmed by the need to continuously scan 
between pictures and text while the auditory channel simultaneously compares incoming streams of spoken words and printed text (Mayer, 2021). In other words, presenting identical information via both information processing channels at the same time rapidly consumes the finite processing capacity of the working memory, overloading the working memory with extraneous processing as learners struggle to make sense of two streams of information in a given moment. When this occurs, valuable cognitive resources are consumed before essential or generative processing can occur, inhibiting meaningful learning via what Mayer (2005c) describes as a redundancy effect.

Studies suggest that the redundancy effect is particularly poignant during faster paced multimedia lessons in which learners cannot control the speed of instruction (Craig et al., 2002; Kalyuga et al., 1999, 2000; Leahy et al., 2003; Jamet \& Le Bohec, 2007; Mayer et al., 2001; Moreno \& Mayer, 2002a; Mousavi et al., 1995). In contrast, in experiments employing interactive lessons where participants were able to progress through instructional content at their own pace, whether through games (e.g., Makransky et al., 2019a; Moreno \& Mayer, 2002b) or slideshows (Mayer et al., 2018), the negative influence of the redundancy effect on learning was decreased. When learners are able to dictate the speed at which instruction occurs, they are provided with an opportunity to more methodically attend to incoming streams of information. Given that this alleviates the need to necessarily process information presented visually and auditorily at the same time, extraneous processing is reduced (Mayer, 2001, 2005c). However, Mayer (2021) cautioned that although redundancy may not diminish learning in slower-paced lessons, the evidence does not suggest that it enhances learning either (e.g., Mayer \& Johnson, 2008). Given this, even in multimedia lessons which learners can complete at a speed of their own choosing, the redundancy principle suggests that instructors should strive to remove redundant printed text from instructional materials (Adesope \& Nesbit, 2012; Mayer, 2001, 
2005c, 2014a, 2021)

The Spatial Contiguity Principle. The spatial contiguity principle suggests that learners understand material better when corresponding words and pictures are presented in close proximity to one another (Mayer, 2001). When corresponding words and pictures are arranged in a manner where they are separated from one another, learners' extraneous processing is exacerbated by the need to scan a given page or screen and determine which pictures are representative of which words, and vice versa. Further, the distance between corresponding words and pictures diminishes learners' ability to hold both pieces of information within the working memory simultaneously, facilitating overload due to increased extraneous processing. In contrast, displaying corresponding pictures and text next to one another provides learners with spatial cues as far as which verbal and nonverbal information are conceptually aligned, allowing learners to conserve cognitive resources which may have otherwise been spent trying to locate words and pictures and interpret their association with one another (Mayer, 2005c, 2014a). Mayer (2005c) suggested that this effect may be even more prevalent when words and pictures are integrated with one another, such that words actually appear within corresponding graphics. When pictures and words are integrated with one another, learners can direct their attention to one specific location within a multimedia lesson in order to obtain a particular piece of information, rather than splitting their attention between multiple information sources (referred to as the split-attention principle from the theoretical perspective of CLT; Ayres \& Sweller, 2014; Sweller et al., 2011). "Separated presentations cause learners to waste limited processing capacity on extraneous processing that could have been used to support the processes of active learning. Thus, separated presentations are less likely to foster understanding than are integrated presentations" (Mayer, 2021, p. 213). Integrating words with pictures can also provide a visual 
representation of how they are conceptually connected to one another, modeling the relationships between words and pictures which learners establish while processing information in the working memory (Mayer, 2001, 2005c). Overall, the spatial contiguity principle suggests that instructors teaching in multimedia environments should present corresponding words and pictures in close proximity to one another, integrating those words and pictures when possible (Mayer, 2005c).

Earlier studies examining the spatial contiguity principle (e.g., Chandler \& Sweller, 1991; Mayer, 1989; Mayer et al., 1995; Sweller et al., 1990; Tindall-Ford et al., 1997) were specifically concerned with how pictures and words were arranged on a given page within a physical workbook or booklet, consistently reporting that learning was enhanced for participants in lessons where pictures and words were presented in close proximity to one another or integrated within one another compared to participants in lessons where pictures and words were presented separately. More recently, this research has been expanded to explore the applicability of the spatial contiguity principle to multimedia lessons incorporating computer-based instruction, demonstrating that the arrangement of graphics (e.g., animations, pictures, diagrams) and words on a computer screen also influences the amount of extraneous processing that learners engage in (e.g., Austin, 2009; Bodemer et al., 2004; Cierniak et al., 2009; Chung, 2007; Florax \& Ploetzner, 2010; Kester et al., 2005; Moreno \& Mayer, 1999a; Johnson \& Mayer, 2012; Makransky et al., 2019b; Pociask \& Morrison, 2008; Schmidt-Weigand et al., 2010). Echoing the sentiments of Ayres and Sweller (2014) and Mayer and Fiorella (2014) in their own reviews, Mayer (2021) synthesized research exploring the spatial contiguity principle across physical and computer-mediated environments and suggested that integrating pictures and words is most applicable when instructional material is complex (e.g., Cerpa et al., 1996; Chandler \& Sweller, 
1996; Sweller \& Chandler, 1994), when visual representations of material are not comprehensible without the inclusion of words (e.g., Chandler \& Sweller, 1991, 1996; Sweller \& Chandler, 1994), and when learners are not already familiar with instructional material (e.g., Mayer et al., 1995; Kalyuga et al., 1998; Yeung et al., 1998). Under such conditions, Mayer (2021) proposed that the spatial arrangement of instructional material guides learners' selection, organization, and integration of information in and of itself, reducing extraneous processing that would otherwise arise as learners attempt to determine how words and pictures correspond with one another independently.

The Temporal Contiguity Principle. The temporal contiguity principle suggests that presenting corresponding words and pictures at the same time, rather than in succession, enhances learning (Mayer, 2001). When words and pictures are presented one after another, leaners are forced to engage in representational holding - "the task of holding all relevant words or images in working memory" (Mayer, 2005c, p. 190) - for whatever span of time might pass between the presentation of a word and the presentation of its corresponding picture. As this occurs, learners must hold words and pictures presented earlier in a lesson within their working memory until all related words and pictures have also been presented later in the lesson. This can quickly overload the finite cognitive capacity of the working memory, which can only hold so many pieces of information (roughly seven; Baddeley, 1997) in a given moment (Mayer, 2001, 2005a, 2014a, 2021; Sweller, 1988; Sweller et al., 2011). Presenting corresponding words and pictures in succession rather than in unison thus makes it more difficult for learners to make conceptual connections between visual and verbal content given that the cognitive resources necessary to make those connections are consumed maintaining mental representations of content. That is, presenting corresponding visual and verbal content successively facilitates 
extraneous processing "instead of taking advantage of our ability to simultaneously process [information] within our visual and verbal channels" (Mayer, 2021, p. 232).

In contrast, the likelihood of learners being able to hold visual and verbal representations of content in working memory is enhanced when corresponding pictures and words are presented at the same time (Mayer, 2005c). When instructors present corresponding visual and verbal materials in tandem, they provide guidance to learners concerning how those materials should be processed, such that "simultaneous presentations prime the learner to build connections between corresponding visual and verbal material" (Mayer, 2021, p. 240). Early studies of temporal contiguity (e.g., Baggett, 1984; Baggett \& Ehrenfeucht, 1981) found that research participants who watched instructional films overlaid with narration performed better on subsequent retention tests than participants in lessons where instructional films and narration were presented successively (regardless of the order in which they were presented). More recent research (e.g., Mayer \& Anderson, 1991; Mayer \& Anderson, 1992; Mayer et al., 1999; Mayer \& Sims, 1994) suggests that the temporal contiguity principle is equally applicable to computer-based educational environments, consistently documenting improved learning for participants presented with corresponding animations and narrations simultaneously compared to participants presented with animations and narrations in succession. Similar to experiments exploring the applicability of the redundancy principle to multimedia lessons in which learners can control the pace of their own instruction (e.g., Mayer et al., 2018; Makransky et al., 2019a; Moreno \& Mayer, 2002b), research suggests that the temporal contiguity principle might also be contingent upon the degree to which learners can engage with instructional materials at a speed of their own choosing (Michas \& Berry, 2000). Specifically, when learners can pause, rewind, and replay sections of multimedia lessons, they are able to revisit information that may have otherwise been 
lost in lessons occurring in real time due to working memory overload (Mayer, 2021).

Nevertheless, empirical evidence lends support to Mayer's (2001, 2005c) notion that temporally integrating visual and verbal instructional materials during multimedia lessons, similar to spatially integrating materials, capitalizes on learners' dual-channel capabilities for information processing in a manner which decreases the likelihood of overloading learners' finite cognitive processing capacity (Ginns, 2006).

\section{Principles for Managing Essential Processing}

The Segmenting Principle. The segmenting principle suggests that multimedia learning is enhanced when instruction is compartmentalized into user-paced, "bite-sized" (p. 171) segments rather than presented as one large, continuous unit (Mayer, 2005b). When instructional content is presented in its entirety to learners within a single lesson, learners may not have sufficient time to appropriately select specific information to pay attention to, organize information into visual or verbal models, or integrate those models. Under such conditions, learners' finite cognitive capacity for processing information impedes their ability to keep pace with comprehensive lessons, potentially causing them to fall behind while making sense of information presented earlier in the lesson or disregard information presented earlier to focus on information presented later. "By the time the learner selects relevant words and pictures from one segment of the presentation, the next segment begins, thereby cutting short the time needed for deeper processing" (Mayer \& Moreno, 2003, p. 47). This is particularly deleterious to learners understanding information when that information is highly complex, and thus high in intrinsic load (Sweller et al., 2011), or when lessons are fast-paced (e.g., Chen \& Yen, 2019) or learners lack prior knowledge related to lesson content (e.g., Ayres, 2006; Spanjers et al., 2011). While it might not be feasible for instructors to reduce the inherent complexity of material, Mayer 
(2005b) suggested that it may nevertheless be possible to assist learners in effectively managing their essential processing via segmenting.

Segmenting refers to "an instructional design technique that is intended to help learners manage essential cognitive load... [breaking] a complex multimedia message into smaller parts that are presented sequentially with pacing under the learners' control" (Mayer, 2021, p. 248). Segmenting is thus comprised of two key features: (1) compartmentalizing a lesson into meaningful sub-units and (2) providing learners with the ability to control the pace of the lesson (Mayer, 2021). In segmenting, "the learner is able to select words and select images from the segment; the learner also has time and capacity to organize and integrate the selected words and images. Then, the learner is ready for the next segment, and so on" (Mayer \& Moreno, 2003, p. 47). Research suggests that this technique is an effective means of assisting learners in managing intrinsic load not only when interpreting instructional materials presented via computer-based presentations and animations (e.g., Boucheix \& Guignard, 2005; Boucheix \& Schneider, 2009; Hasler et al., 2007; Hassanabadi et al., 2011; Mautone \& Mayer, 2007; Mayer \& Chandler, 2001; Mayer et al., 2003; Mayer et al., 2018; Mayer et al., 2019; Stiller et al., 2009; Sung \& Mayer, 2013) or informational videos (e.g., Biard et al., 2018; Ibrahim et al., 2012; Moreno, 2007), but also when completing worked-examples (e.g., Ayres, 2006; Catrambone, 1994, 1995, 1998; Gerjets et al., 2004, 2006) or participating in simulated game-based learning (e.g., Lee et al., 2006). Indeed, the availability of consistent empirical support for the segmenting principle across multimedia contexts led the authors of a recent meta-analysis to conclude that "multimedia instructions should be presented in (meaningful and coherent) learner-paced segments, rather than as continuous units, to improve learning performance and reduce the learners' overall cognitive load" (Rey et al., 2019, p. 415). By doing so, instructors in multimedia environments 
can assist their students in managing essential cognitive processing effectively as they engage with, and make sense of, complex information (Mayer, 2005b).

Reviewing studies appraising pacing as a component of clear teaching (e.g., Bush et al., 1977; Chesebro, 2003; Chesebro \& McCroskey, 1998; Cruickshank, 1985; Hines et al., 1985; Kennedy et al., 1978), Bolkan (2017a) suggested that incorporating appropriate pace into the organizational structure of a lesson is critical given that "if students cannot keep up with course materials, they cannot comprehend their lessons" (p. 23). Based on this rationale, Bolkan (2017a) incorporated the extent to which instructors engage in segmenting (e.g., "breaking lectures into specific and manageable content blocks," "teaching one step at a time" [p.28]) into his operationalization of structure; one of the five dimensions he ultimately suggested comprise the construct of teacher clarity. Similar to the ways in which superfluous information and vague explanations impede students' ability to effectively process information, the rate at which information is presented to students can also negatively impact their learning by overloading the finite processing capacity of the working memory (Mayer, 2005b). From a communicative perspective, segmenting constitutes a critical component of clear teaching given its impact on students' ability to effectively select, understand, and remember instructional content.

The Pre-Training Principle. The pre-training principle refers to the idea that learners are able to process information conveyed via multimedia messages more deeply when they are already familiar with names, characteristics, and other main concepts presented in those messages (Mayer, 2005b). Recall that the theoretical basis for the segmenting principle is that learners exposed to complex or fast-paced presentations of instructional content can quickly become overwhelmed while trying to construct mental models. In some situations, presentations can be compartmentalized via segmenting in order to avoid overloading learners' finite 
information processing capacity, conserving cognitive resources which may then be committed to essential and generative processing. However, not every multimedia lesson can necessarily be broken down into the "bite sized" (p. 171), self-contained units described by Mayer (2005b) due to a variety of different constraints (e.g., time, technology). In such situations, Mayer (2005b) suggested that a viable alternative may be to provide learners with prior instruction related to the fundamental concepts to be presented in a lesson before attempting any explanations as far as how those concepts are interlinked - a strategy which he described as pre-training.

In constructing a mental model, learners must develop an understanding of (1) how each component of the model works in-and-of-itself and (2) how each component of the model, or changes in any component of the model, affect the rest (Mayer et al., 2002a). Via pre-training, instructors provide students with explanations concerning how each component of a model functions on its own prior to presenting information regarding how components relate to one another, eliminating the need for learners to process information related to both simultaneously. In doing so, instructors decrease the amount of intrinsic load placed upon the working memory, lessening the extent to which learners must engage in essential processing. Given the additive relationship between extraneous and essential processing (Sweller et al., 2011), pre-training thus constitutes an instructional technique which can facilitate greater space in learners' working memory for generative processing conducive to meaningful learning (assuming that instructors are also teaching in ways which reduce extraneous load). Experimental research comparing differences in learning outcomes between participants exposed to a single lesson versus participants exposed to lessons comprised of multiple phases offer consistent support for the pretraining principle, repeatedly demonstrating that learners perform better on post-lesson assessments when provided with prior instruction (e.g., Clark et al., 2005; Eitel et al., 2013; 
Fiorella \& Mayer, 2012; Gegner et al., 2009; Kester et al., 2006; Mayer, 1979a, 1979b, 1983; Mayer et al., 2002; Mayer et al., 2002b; McCrudden et al., 2011; Pollock et al., 2002). Several of these studies (e.g. Clark et al., 2005; Pollock et al., 2002) lend further empirical support to the pre-training principle insofar as the positive influence of pre-training on participants' learning was only observed for participants lacking prior knowledge related to lesson content, such that high-experience learners exhibited less overload attributable to essential processing. Given this, pre-training may be an effective strategy for instructors to use in multimedia environments when attempting to assist students in managing essential processing while unable to incorporate segmenting into their instructional design (Mayer, 2005b, 2014a, 2021).

The Modality Principle. The modality principle suggests that learners understand material better when it is presented through the combination of pictures and spoken words rather than through the combination of pictures and printed words (Mayer, 2001). When instructors in multimedia environments present learners with information using pictures and printed words (i.e., text), the entirety of that information is detected by learners' eyes and thus poses a risk of overloading the visual information processing channel. Specifically, as long as learners are looking at a picture they are unable to read its accompanying text, and as long as learners are reading text they are unable to look at its accompanying picture. When this occurs, learners experience similar split-attention effects to those arising from a lack of spatial contiguity, such that the need to continuously look back and forth between pictures and text facilitates greater representational holding and, in turn, greater levels of both essential and extraneous processing. Given this, Mayer (2005b) recommended that instructors in multimedia environments should strive to decrease the amount of information learners must process visually by engaging in modality offloading - presenting verbal information in the form of narration rather than readable 
text. In doing so, Mayer (2005b) argued that instructors assist learners in effectively managing essential processing demands by shifting some of the intrinsic load associated with a given learning task to the auditory channel rather than relying exclusively upon visual processing. Modality offloading is thus an instructional strategy which capitalizes upon the innate dualchannel processing system of the brain (Mayer, 2021).

Modality offloading has been the focus of a litany of studies, resulting in the modality principle emerging as one of the most empirically supported principles of effective instructional in multimedia contexts (Mayer, 2021). While earlier research explored the impact of modality offloading on learning in lessons entailing paper-based instruction (e.g., Leahy et al., 2003; Mousavi et al., 1995; Tindall-Ford et al., 1997), more recent research assessing modality offloading in computer-based contexts has consistently documented improved learning outcomes for participants exposed to lessons comprised of corresponding graphics (e.g., animations, illustrations) and narration compared to participants in lessons based on corresponding graphics and on-screen text (e.g., Atkinson, 2002; Craig et al., 2002; Harskamp et al., 2007; Jeung et al., 1997; Kalyuga et al., 1999; Lee \& Mayer, 2018; Mayer et al., 2003; Mayer \& Moreno, 1998; Mayer et al., 2019; Moreno \& Mayer, 1999a, 2002b; Moreno et al., 2001). Similar to the redundancy principle, temporal contiguity principle, and segmenting principle, research suggests that the modality principle is particularly applicable in scenarios where the speed of instruction is fast-paced (e.g., Tabbers et al., 2004) or the length of instruction is long (e.g., Leahy \& Sweller, 2011; Wong et al., 2012). This is consistent with the predictions of CTML given that modality offloading is a strategy designed to assist learners overwhelmed by intrinsic and extraneous cognitive load simultaneously during lessons. When instruction is slow or learners are given opportunities to stop, rewind, or replay portions of a multimedia lesson, there is less likelihood of 
the working memory being overloaded even when information is processed exclusively through the visual channel. Given this, the modality principle may be most useful for instructors teaching multimedia lessons which are synchronous as opposed to asynchronous, given that learners are less able to exercise control over the pacing of live lessons. Regardless, meta-analyses synthesizing research examining both the influence and boundary conditions of the modality principle consistently characterize modality offloading as best instructional practice for teachers in multimedia environments (e.g., Ginns, 2005; Low \& Sweller, 2014; Moreno, 2006; Reinwein, 2012).

\section{Principles for Fostering Generative Processing}

The Multimedia Principle. The multimedia principle, as referenced previously in this manuscript, suggests that learners understand instructional content better when it presented using words and pictures rather than words alone (Mayer, 2001). Recall that CTML (Mayer, 2001) is based on three central assumptions regarding how human beings learn: (1) human beings use different processing channels to make sense of information that they see and hear (i.e., the dualchannel assumption), (2) there is a cap on the amount of information that the human brain can process via the visual and auditory channels at a single point in time (i.e., the limited capacity assumption), and (3) human beings must actively engage in their own cognitive processing in order to effectively construct coherent and meaningful mental representations of information presented through the visual or auditory channels (i.e., the active processing assumption). Given these assumptions, words and pictures - though they might correspond with one another in referring to the same concept or topic during a given lesson - are not informationally equivalent from a CTML perspective (Mayer, 2001, 2005a). Rather, “words and pictures prime two qualitatively different knowledge representation systems in learners” (Mayer, 2021, p. 121). 
Fletcher and Tobias (2005) suggested that instructors "should capitalize on the availability of the different input channels that are available for cognitive processing" (p. 124) by incorporating both pictures and words into their teaching, as "using the same modality for all of the material being presented... may lead to channel overload and reduce learning” (p. 124). Further, as extraneous processing is reduced and essential processing is managed effectively, learners retain greater cognitive resources which can be used for deeper and more meaningful learning via generative processing (Mayer, 2001, 2005a, 2014a, 2021). Based on the multimedia principle, Mayer (2021) ultimately suggested that instructors should be less concerned with asking questions such as which medium for instruction is best and more concerned with how visual and auditory mediums can be used simultaneously to enhance student learning.

Empirical support for the multimedia principle has been comprehensive and consistent, with research exploring multimedia presentations based on both physical (e.g., Mayer, 1983, 1989; Mayer \& Gallini, 1990; Mayer et al., 1996; Stull \& Mayer, 2007) and computer-based (e.g., Chun \& Plass, 1996; Jones \& Plass, 2002; Lee \& Mayer, 2015; Mayer \& Anderson, 1991, 1992; Mayer et al., 2014; Moreno \& Mayer, 1999b, 2002a; Plass et al., 1998; Ponce \& Mayer, 2014b; Schmeck et al., 2014) materials providing compelling evidence that learners understand instructional content better when it is presented using both words and pictures. While multimedia effects observed across these studies are largely contingent upon the degree to which teaching is aligned with other principles of multimedia instructional design (e.g., the coherence principle, the signaling principle, contiguity principles, etc.), they nevertheless overwhelmingly lend credence to Mayer's (2001) contention that learning is enhanced when information is presented through the combination of pictures and words. An overarching goal of CTML is thus to provide instructors with guidance insofar as how to most effectively employ pictures and words while 
teaching (Mayer, 2001, 2005a, 2014a, 2021).

The Personalization Principle. The personalization principle is that learners understand instructional content in multimedia lessons better when it is presented conversationally rather than formally (Mayer, 2005d). While the extent to which generative processing occurs is largely dependent on learners' available cognitive capacity subsequent to extraneous and essential processing, generative processing is also enhanced when learners are motivated to engage with instructional material (Mayer, 2005d, 2014a, 2021). Recall that CTML (Mayer, 2005a) characterizes learning as highly deliberate and cognitively intensive, describing learners as “active [information] processors who seek to make sense of multimedia presentations" (p. 36). The cognitive processes associated with selecting, organizing, and integrating novel information necessitate learners' active engagement with instructional content, placing strenuous demands on learners' limited cognitive resources as they attempt to determine which information is relevant, construct coherent mental representations of that information, and create meaningful connections between that information and prior knowledge. Put simply, learning requires effort, and learners are more likely to put forth greater effort in scenarios where they are sufficiently motivated to do so (Mayer, 2021). Given this, Mayer (2005d) suggested that instructors in multimedia environments seeking to facilitate students' generative processing may be able to do so through the personalization of instructional content, eliciting greater motivation from students to participate in the learning process.

Two specific strategies which instructors might use to personalize their multimedia lessons are (1) incorporating personally inclusive language into lessons such as "you" and "I", or (2) integrating comments or statements into lessons through which they directly address a specific learner or group of learners (Mayer, 2005d). Using these techniques, instructors 
manipulate social cues in a manner that acknowledges both themselves and their students as individuals, making lessons more conversational to increase learners' sense of social presence and cultivate learners' perceptions that they have a personal relationship with their instructor. Complementing Mayer's (2005d) rationale, an abundance of instructional communication research has consistently demonstrated that the extent to which students identify with their instructors, perceive they have rapport with their instructors, or otherwise feel positively about their instructors can enhance their learning in courses and lessons taught by those instructors (e.g., Allen et al., 2006; Frisby \& Martin, 2010; Goodboy et al., 2018; Rodríguez et al., 1996), catalyzing significant interest in relational aspects of teaching among instructional communication scholars (Farris et al., 2018; Mottet \& Beebe, 2006; Mottet et al., 2006). Further, Mayer's (2005d) definition of personalization appears to have some conceptual overlap with Gorham's (1988) description of verbal immediacy, through which teachers demonstrate "availability to engage in one-on-one interactions" (p. 52) with students and emphasize their "humanness" (p. 52) via verbal behaviors such as self-disclosure, praise, humor, feedback, asking questions, and - in particular - inclusive language. Mayer (2005d), though not identifying as a member of the instructional communication discipline, suggested that the relationship between instructor-student relationships and learning might occur largely due to social agency. Specifically, Mayer (2005d) proposed that "Social cues in multimedia instructional messages can prime a social response in learners that leads to deeper cognitive processing and better learning outcomes" (p. 202), particularly for learners who may otherwise be unmotivated to actively engage in the cognitively intensive tasks associated with the learning process.

Numerous experiments suggest that personalizing multimedia lessons can enhance learning outcomes, exploring the impact of communicating conversationally with students via 
narrations (e.g., Mayer et al., 2004; McLaren et al., 2011a, 2011b; Moreno \& Mayer, 2000) and text (e.g., Dutke et al., 2016; Ginns \& Fraser, 2010; Moreno \& Mayer, 2000). Studies have also examined how personalized lessons presented by fictional characters or on-screen agents can influence learning (e.g., Moreno \& Mayer, 2000, 2004; Wang et al., 2008), as well as tested the applicability of the personalization principle to multimedia lessons in which learners speak different languages or belong to different cultures (e.g., Ginns \& Fraser, 2010; Kartal, 2010; Reichelt et al., 2014; Rey \& Steib, 2013; Schrader et al., 2018; Schworm \& Stiller, 2012). Generally, this research has consistently demonstrated that learners understand instructional content better when it is presented to them conversationally rather than formally, although certain studies do suggest that personalization may not improve learning when learners are already familiar with the subject matter presented in a given lesson (e.g., Stiller \& Jedlicka, 2010), when lessons are unnecessarily long (e.g., Ginns et al., 2013), or when instructors are too overzealous in their attempts to personalize their lessons and ultimately incorporate seductive details conducive to extraneous processing (e.g., Mayer et al., 2004). Overall, research suggests that instructors in multimedia environments can facilitate greater active engagement and, therefore, more generative processing from learners by personalizing the lessons that they teach (Mayer, 2021).

The Voice Principle. Similar to the personalization principle, the voice principle also incorporates instructors' use of social cues into a CTML framework for facilitating generative processing (Mayer, 2005d). Specifically, the voice principle is that learners understand information from multimedia lessons better when lessons are narrated by "an appealing human voice" (Mayer, 2021, p. 322). Mayer (2005d) wrote that "a human voice, speaking with a standard accent conveys the idea that someone is speaking directly to you... a machine- 
synthesized voice - although perceptually discernable - may convey less of a sense of social presence" (p. 204). That is, learners in multimedia lessons narrated by a human voice are more likely to perceive their instructor as an actual person speaking directly to them than learners in lessons narrated by computers (e.g., Atkinson et al., 2005; Mayer \& DaPra, 2012; Mayer et al., 2003). When learners perceive an instructor as a human being and conversational partner, they exert greater effort in trying to make sense of what that instructor is trying to say (e.g., Mayer, 2005d, 2014a, 2021). Given this, Mayer (2005d) suggested that instructors teaching multimedia lessons may be able facilitate learners' generative processing by providing human rather than computer-synthesized narrations of instructional content, arousing a greater sense of social presence among learners conducive to enhanced motivation to engage in deeper information processing.

Interestingly, Mayer (2005d) suggested that the voice principle may also facilitate different outcomes for learners participating in multimedia lessons narrated by human instructors with different accents, writing that "a human voice with a foreign accent - such as a Russian accent - may also diminish the learner's social response to the message" (p. 204). Reviewing empirically-based research exploring the influence of instructor accents on learning (e.g., Domagk, 2010; Mayer et al., 2003), Mayer (2021) reiterated the importance of learners finding whatever voice narrates a given multimedia lesson appealing (i.e., attractive, interesting), describing an appealing voice as "a voice that conveys a positive social connection between the learner and the instructor" (p. 329). However, which specific accents learners find appealing or unappealing is likely highly subjective, varying between individual learners. In fact, research in instructional communication suggests that certain students sometimes perceive their instructors' foreign accents as so unappealing that they characterize those accents as instructor misbehaviors 
(i.e., teaching behaviors which interfere with instruction and learning; Goodboy \& Myers, 2015; Kearney et al., 1991). Given this, instructors seeking to incorporate the voice principle into their multimedia lessons might err on the side of caution by narrating lessons using accents which are as similar to their learners' accents (i.e., a "standard accent," Mayer, 2005d) as possible, decreasing the likelihood of learners perceiving that accent as unappealing. However, given the relatively few studies which have explored the influence of instructor voice and accent on multimedia learning to date, Mayer (2021) ultimately concluded that further research is warranted before advocating the voice principle as best practice for multimedia instruction.

The Image Principle. The image principle, originally, was that learners understand instructional material better when their instructors are visible on-screen during multimedia lessons (Mayer, 2005d). Similar to the rationale supporting the personalization and voice principles, Mayer (2005d) argued that instructors in multimedia contexts might be better able to facilitate generative processing from learners by stimulating their sense of social presence, thereby arousing increased motivation to engage with instructional content. Specifically, Mayer (2005d) suggested that “A seemingly straightforward way to increase the learner's sense of social presence is to add an on-screen character who appears to interact with the learner" (p. 204), reasoning that being able to see an instructor (or instructional agent) would inherently enhance learners' likelihood of identifying with and relating to that instructor. However, Mayer (2014a) later revised the image principle based on inconsistent and contradictory findings reported across numerous empirical studies (e.g., Atkinson, 2002; Lusk \& Atkinson, 2007; Colliot \& Jamet, 2018; Craig et al., 2002; Domagk, 2010; Frechette \& Moreno, 2010; Li et al., 2020; Moreno et al., 2001; Mayer et al., 2003; Mayer \& DaPra, 2012; Park, 2015; Wang et al., 2018), delineating the image principle from the embodiment principle (described next) by 
emphasizing that the image principle refers exclusively to the inclusion of (relatively) static images of instructors during multimedia lessons - such as illustrations, photographs, or talkingheads (i.e. videos or animations of instructors viewed in close-up with relatively little movement). That is, the revised image principle refers only to whether learners can specifically see their instructors during multimedia lessons (Mayer, 2014a, 2021).

Counter to the original image principle (Mayer, 2005), Mayer (2014a) suggested that learners do not necessarily understand instructional material better when a static image of an instructor is added to a multimedia lesson - going so far as to suggest that including a static image of an instructor may ultimately inhibit learning by increasing extraneous load. In particular, "The static image of an instructor on the screen may be distracting or somewhat creepy because it does not display human-like movement, eye-gaze, and gesture. This can violate the coherence principle and thereby create extraneous processing” (Mayer, 2021, p. 331). At best, the inclusion of static, or low-embodied (i.e., relatively unmoving or unanimated), images of instructors in multimedia lessons serves as a seductive detail that may superficially arouse immediate attention but distract learners from important information, wasting limited cognitive capacity that could have otherwise been used for essential or generative processing (e.g., Stull et al., 2018). At worst, static and low-embodied images of instructors might actually undermine learners' sense of social presence by making learners feel uncomfortable during multimedia lessons (Mayer, 2021).

The Embodiment Principle. The embodiment principle, originally encapsulated within the image principle (Mayer, 2005d), is that learners understand multimedia lessons better when they can see an instructor or on-screen instructional agent who engages in humanlike body movement, gestures, eye contact, and facial expressions (Mayer, 2014a). As articulated in the 
revised image principle (Mayer, 2014a), simply being able to see an image of an instructor is not necessarily enough to cultivate a sense of social presence among learners in multimedia lessons. Rather, social presence is aroused in scenarios when learners can observe their instructors engaging in behaviors which Mayer (2014a) described as high-embodiment (i.e., behaviors similar those used during in-person interactions in the real world). Elaborating further, Mayer (2021) wrote that examples of high-embodiment "include using hand gestures while talking... maintaining eye contact while talking... drawing graphics by hand while talking... or manipulating objects from a first-person perspective," ultimately characterizing highembodiment behaviors as "the ways that onscreen instructors can use their bodies to enhance the act of instructional communication" (p. 344). When instructors in multimedia lessons engage in high-embodiment behaviors, Mayer (2021) explained that they provide "a positive social cue that primes a sense of social partnership in the learner, causing the learner to try harder to understand the instructional message and thereby learn more deeply" (p. 341). That is, when instructors are nonverbally animated and employ movement during multimedia lessons, they can motivate learners to engage in generative processing conducive to meaningful learning.

Unlike Mayer's (2005d) original articulation of the image principle, experimental research (e.g., Baylor \& Kim, 2009; Dunsworth \& Atkinson, 2010; Lusk \& Atkinson, 2007; Moreno et al., 2010) has consistently reported findings which lend empirical support for the embodiment principle (2014a). Specifically, studies have found that the extent to which instructors engage in nonverbal gestures (e.g., Li et al., 2019; Mayer \& DaPra, 2012; Wang et al., 2018), maintain eye contact with learners (e.g., Fiorella et al., 2019, 2020; Stull et al., 2018), physically draw graphics (e.g., Fiorella \& Mayer, 2016a), and move from a first-person perspective (e.g., Fiorella et al., 2017) during multimedia lessons can significantly enhance 
learners' performance on post-lesson assessments of both retention and transfer. Although research suggests that other aspects of a multimedia lesson might diminish these effects - such as the presence of unappealing voice (e.g., Mayer \& DaPra, 2012), the use of general or vague pointing rather than pointing at specific images and words (e.g., Li et al., 2019), or learners not being able to see an instructor's hand as he or she draws a graphic (e.g., Fiorella \& Mayer, 2016a; Fiorella et al., 2019, 2020) - the findings of experimental studies generally suggest that instructors' high-embodiment behaviors can increase learning in multimedia lessons. Altogether, the available literature generally suggests that learners' social presence is enhanced when their instructors are nonverbally animated, catalyzing social responses conducive to motivating learners to engage in generative processing (Mayer, 2021).

Much like the conceptual overlap between Mayer's (2005d) personalization principle and Gorham's (1988) description of verbal immediacy, Mayer's (2014a) notion of embodiment is similar to Andersen's (1979) definition of nonverbal immediacy (i.e., "nonverbal behaviors that reduce physical or psychological distance between teachers and students" [p. 543]). Instructional communication research has suggested that instructors who engage in nonverbal immediacy behaviors (e.g., smiling, making eye contact, moving around the classroom) enhance their students' motivation (Frymier, 1994), participation (Rocca, 2009), and positive affective experiences during learning (Witt et al., 2004). In particular, nonverbal immediacy has been suggested to enhance teacher-student relationships (Frymier \& Houser, 2000) in a manner which satisfies students' innate relational needs (Mottet et al., 2006), thereby motivating students to engage more deeply with instructional content (Frymier et al., 2019). Mayer's (2014a) suggestion that instructors' high-embodiment behaviors (e.g., body movement, gestures, eye contact, and facial expressions) can arouse learners' sense of social presence, increase 
motivation, and facilitate generative information processing during multimedia lessons thus seems consistent with instructional communication literature concerning the influence of instructor nonverbal immediacy. More recent research exploring instructors' use of nonverbal immediacy behaviors in online teaching (e.g., Dixson et al., 2017; Ramlatchan \& Watson, 2020) lend further credence to Mayer's (2014a) arguments, demonstrating that the implications of instructors' nonverbal behaviors may not be exclusive to face-to-face instruction.

The Immersion Principle. The immersion principle was recently proposed by Mayer (2021) while exploring how CTML-based principles of instructional design might apply to teaching in virtual reality environments. Specifically, the immersion principle is that learners do not necessarily understand instructional content better when it is taught in 3-Dimensional (3D) virtual reality than they do when it is taught using more traditional 2-Dimensional (2D) lessons and presentations. In particular, Mayer (2021) suggested that although multimedia lessons incorporating virtual reality technologies may stimulate learners' sense of presence (i.e., "feeling of being in a place that is artificially created," p. 359) in the short-term, the attention-grabbing and interest-catching features of virtual reality environments might also divert learners' limited cognitive processing capacity away from important instructional content as they engage with virtual sensory and motor distractions.

Recall that one of the central assumptions of CTML (Mayer, 2001) is that learners are only able to process so much information through the visual and auditory channels at a single point in time. Highly immersive virtual reality environments present learners with an abundance of sensory signals which must be continuously processed through the eyes and ears, thereby creating the sensation that the virtual world is real. Given this, Mayer (2021) wrote that although “the perceptual realism of immersive virtual reality may increase the learner's emotional 
response and feeling of presence" (p. 357), the amount of details presented via virtual reality environments might ultimately "create extraneous processing that distracts the learner from the core material of the lesson" (p. 357) - violating CTML's coherence principle (Mayer, 2001). Game and simulation-based learning employing virtual reality technologies - though initially interesting - may thus ultimately inhibit learners' effective use of cognitive resources, facilitating extraneous processing as learners focus on interacting with a virtual environment instead of deeply engaging with instructional content presented through that environment (Mayer, 2021).

Given the potential for 3D virtual reality technologies to both stimulate interest and overload visual and auditory channels for information processing, research has reported mixed findings as far as the extent to which virtual reality environments are conducive to student learning. Though few in number, studies exploring the relationship between virtual reality instruction and learning (e.g., Makransky et al., 2019; Makransky et al., 2019a; Moreno \& Mayer, 2002b, 2004; Parong \& Mayer, 2018) have reported inconsistent conclusions regarding whether virtual reality enhances or diminishes learning, reminiscent of Mayer's (2014a) revised image principle of instructional design. Given that virtual reality is a relatively recent technological development and thus has not been extensively studied as an instructional tool, Mayer (2021) ultimately concluded that more research is necessary before attempting to make any definitive statements concerning how instructors' use of virtual reality might influence students' learning. However, the empirical evidence which is currently available does not appear to suggest that multimedia lessons employing 3D virtual reality technologies are any more effective than lower immersion 2D lessons when it comes to facilitating generative processing and learning. 
The Generative Activity Principle. The generative activity principle suggests that learners understand instructional content better when their instructors take the time to guide them through specific activities which stimulate generative processing (Mayer, 2021). Given that CTML (Mayer, 2001) is based on the assumption that meaningful learning is an active process, Mayer (2021) characterized activities which directly prompt learners to engage in active learning as inherently conducive to generative processing. Specifically, when instructors provide learners with opportunities to summarize, map, draw, imagine, self-test, self-explain, teach, or enact instructional content, they directly stimulate learners' selection of important information, organization of information into coherent mental representations, and integration of mental representations with prior knowledge already stored within the long-term memory (Mayer, 2021). Generative activities thus refer to specific tasks that learners engage in during multimedia lessons with the intention of promoting deeper, more meaningful learning. Summarizing instructional content, for example, is a generative activity in that it requires learners to "select information to put into [their] summary, organize it into a coherent set of sentences, and integrate it with prior knowledge by putting it in [their] own words" (Mayer, 2021, p. 372). Similarly, mapping (i.e., creating spatial representations of important information or terms), drawing (i.e., creating illustrations which depict instructional content), imagining (i.e., creating mental images which depict instructional content), self-testing (i.e., studying information and completing practice assessments), self-explaining (i.e., creating one's own explanations for instructional content), teaching (i.e., providing other learners with explanations of instructional content), and enacting (i.e., physically moving corporeal objects to act out instructional content) encourage learners to reflect more deeply on information presented in multimedia lessons in ways which stimulate generative processing (Mayer, 2021). Generative activities are thus a mechanism 
through which instructors can "clearly guide and scaffold the learner's generative activity" (Mayer, 2021, p. 376), assuming that they have already employed an instructional design conducive to reducing extraneous processing and assisting learners in effectively managing essential processing.

Mayer's (2021) generative principle is well-supported by empirical research (e.g., Fiorella \& Mayer, 2015, 2016b). Indeed, a vast array of experimental studies have consistently demonstrated that learners' generative processing is enhanced in multimedia lessons where they are directly prompted to engage in generative activities - whether by summarizing (e.g., Parong \& Mayer, 2018; Peper \& Mayer, 1978, 1986; Ponce et al., 2018; Shrager \& Mayer, 1989), mapping (e.g., Fiorella \& Mayer, 2017; Ponce \& Mayer, 2014a, 2014b; Ponce et al., 2018; Stull \& Mayer, 2007), drawing (e.g., Fiorella et al., 2020; Schmeck et al., 2014; Schwamborn et al., 2010) imagining (e.g., Leopold \& Mayer, 2015; Leopold et al., 2019), self-testing (e.g., Johnson \& Mayer, 2009; Mayer, 1975, 1980; Sagerman \& Mayer, 1987), self-explaining (e.g., Fiorella \& Mayer, 2012; Johnson \& Mayer, 2010; Mayer, 1980; Mayer \& Johnson, 2010; Pilegard \& Mayer, 2016; Fiorella et al., 2020), teaching (e.g., Fiorella \& Mayer, 2013, 2014), or enacting (e.g., Fiorella et al., 2017; Mayer, 1976; Mayer et al., 2002; Moreno \& Mayer, 1999b). Although Mayer (2021) urged instructors in multimedia contexts to be cautious as far as prompting learners to engage in generative activities before they are ready (i.e., when information is highly complex [e.g., Leopold et al., 2019] or learners' lack sufficient foundational understanding or skill [e.g., Fiorella \& Mayer, 2012; Moreno \& Mayer, 1999b]) or in a manner which learners might perceive as tedious (e.g., Johnson \& Mayer, 2010; Stull \& Mayer, 2007), he nevertheless concluded that generative activities may represent the most direct pedagogical strategy through which instructors can stimulate learners' generative processing in multimedia lessons (assuming 
that extraneous and essential processing demands have already been sufficiently reduced).

\section{Rationale}

Given the widespread transition to online instruction which took place across higher education in the wake of the COVID-19 pandemic (Carillo \& Flores, 2020; Murphy, 2020; Stafford, 2020), the purpose of this dissertation was to explore how instructors adapting to teaching remotely might employ CTML principles of effective instructional design in creating and teaching their multimedia lessons. In particular, this dissertation examined how instructors might utilize specific features of Zoom (given its popularity as a remote teaching platform; Jones, 2020; Kristóf, 2020; Rapanta et al., 2020; Stafford, 2020) to enact CTML principles, thereby designing multimedia lessons in a manner conducive to students' meaningful learning. Of the 15 CTML principles reviewed, three in particular seem especially applicable while teaching via Zoom insofar as they align with specific Zoom features: (1) the signaling principle, (2) the embodiment principle, and (3) the generative activity principle.

The signaling principle - that "people learn more deeply from a multimedia message when cues are added that highlight the organization of essential material" (Mayer, 2005c, p. 184) - seems particularly applicable to online lessons in which instructors utilize Zoom's annotation function to draw students' attention to specific instructional content. As instructors use Zoom's screen-share feature to present content displayed on their own computer screens to students, the annotation function allows them to draw various graphics (e.g., lines, arrows, squares, circles), place "stamps" (e.g., check marks, stars, arrows, exes), and employ "spotlights" to point at, highlight, or otherwise emphasize particular information (Zoom, n.d.). In these ways, Zoom's annotation feature affords instructors the ability to engage in signaling, providing a potential means by which instructors using Zoom can assist students in selecting information relevant to 
instructional goals.

The embodiment principle - that learners understand multimedia lessons better when they can see an instructor or on-screen instructional agent who engages in humanlike body movement, gestures, eye contact, and facial expressions (Mayer, 2014a) - can be implemented into Zoom teaching as simply as plugging in and activating one's camera. Zoom's default settings make displaying oneself to students a straightforward process (Zoom, n.d.). Upon activating their cameras, instructors become visible to all students connected to a given Zoom chatroom, and instructors remain visible when using Zoom's screen-share feature (displayed in a smaller window in the corner of their students' screens). Zoom's inherent design thus enables students to see their instructors throughout an online lesson, providing instructors with the opportunity to engage in the high-embodiment behaviors which Mayer (2014a) characterized as conducive to students' sense of social presence and generative processing.

Lastly, the generative activity principle - that learners understand instructional content better when their instructors take the time to guide them through activities which stimulate generative processing (Mayer, 2021) - can also be directly incorporated into online teaching using specific Zoom features. Polling, in particular, seems especially conducive to prompting students in online lessons to engage in the generative activities which Mayer (2021) suggested prime active learning and generative processing. Polling provides instructors with a means of soliciting student input or posing content-related questions to a class. Given this, instructors teaching via Zoom might use the program's polling feature to provide students with an opportunity to self-test their understanding of recently presented information, scaffolding students' generative activity to facilitate appropriate selection, organization, and integration of instructional content. 
In these ways, it is feasible that instructors teaching via Zoom might use specific program features strategically to incorporate the signaling, embodiment, and generative activity principles into their synchronous (i.e., live) online teaching - enhancing instructional clarity by using Zoom features to assist learners in selecting, understanding, and retaining information (Titsworth \& Mazer, 2016). In contrast, Zoom features seem less conducive to implementing CTML principles into asynchronous (i.e., pre-recorded) online teaching. Polling, for example, is designed to prompt feedback and participation from users during ongoing presentations (Zoom, n.d.). Inherently, then, the use of this feature is only applicable in live lessons where students are presented with instructional content simultaneously. Given the extent to which Zoom's features are designed to stimulate student activity in real-time (Zoom, n.d.), they are more aligned with synchronous, rather than asynchronous, online teaching.

The ability to solicit student feedback or prompt discussion among students mid-lesson has been repeatedly identified as an important feature which distinguishes synchronous from asynchronous online instruction (McBrien et al., 2009; Offir et al., 2008; Peterson et al., 2018). While synchronous teaching provides teachers and students with opportunities for immediate personal engagement and substantive discussion as instruction unfolds (McBrien et al., 2009), asynchronous teaching involves delayed social exchanges frequently characterized by lack of participant involvement (e.g., Hewitt, 2005; Hew et al., 2010). Further, research suggests that asynchronous teaching - in general - may not necessarily be as engaging for students as synchronous teaching. Kuznekoff (2020), for example, reported that students in a recent study only watched about $60 \%$ of an asynchronous video lesson, with only $34 \%$ of students potentially watching the entire lesson from start to finish. In an earlier study, Guo and colleagues (2014) reported that the median amount of time which students spent watching a 9-12 minute video was 
between six and seven minutes, and the amount of time which students spent watching videos ranging from 12 to 40 minutes in length was only between three and four minutes. Given the centrality of student engagement in the active sense-making processes which Mayer (2001) suggested drive meaningful learning experiences, teaching synchronously may be more preferable than teaching asynchronously from a CTML perspective.

In light of the abundant empirical research already suggesting that implementation of the signaling (e.g., Bolkan, 2017b; Harp, \& Mayer, 1998; Li et al., 2019; Loman \& Mayer, 1983; Mautone \& Mayer, 2001; Mayer et al., 1984), embodiment (e.g., Li et al., 2019; Mayer \& DaPra, 2012; Wang et al., 2018), and generative activity principles (e.g., Fiorella \& Mayer, 2013, 2014; Parong \& Mayer, 2018; Peper \& Mayer, 1978, 1986; Ponce et al., 2018; Shrager \& Mayer, 1989) can significantly enhance students' learning in multimedia lessons, it was anticipated that instructors' efforts to incorporate these principles using specific Zoom features would similarly enhance student learning. Given that one of the fundamental purposes for which CTML (Mayer, 2001) was developed was to guide instructors in designing their multimedia presentations in ways which do not overwhelm learners' finite information processing capacity, it was expected that students in an online lesson where an instructor enacts CTML-based principles of instructional design should experience less working memory overload than in an online lesson where an instructor does not. That is, the first hypothesis of this dissertation predicted that students participating in a Zoom lesson with an instructor who strategically uses Zoom features to enact the signaling, embodiment, and generative activity principles would experience less working memory overload than students in a Zoom lesson with an instructor who does not.

H1: $\quad$ Compared to a standard synchronous lesson without Zoom features, students will experience less working memory overload when their instructor uses Zoom 
features to enact the signaling, embodiment, and generative activity principles during a synchronous Zoom lesson.

Recall that CTML (Mayer, 2001) characterizes meaningful learning as an active process. Specifically, CTML is grounded in the assumption that learning occurs when individuals appropriately (1) select relevant visual or auditory information to pay attention to, (2) organize that information into concrete mental representations within the working memory, and (3) integrate those mental representations with prior knowledge already stored in the long-term memory (Mayer, 2001, 2005a, 2014a, 2021). From a CTML perspective, learners' selection, organization, and integration of novel information thus constitute the causal mechanisms through which learning ultimately occurs, providing a theoretical blueprint of the step-by-step process that learners engage in while making sense of new information. As instructors implement CTML principles to create multimedia lessons in ways which account for the finite information processing capacity of the working memory, they ultimately serve as guides who assist students in navigating this process (Mayer, 2001, 2005a, 2014a, 2021). Given this, instructors who strategically use Zoom features to incorporate CTML-based principles into their online lessons should stimulate students' overall learning by reducing working memory overload in a manner which enhances students' selection, organization, and integration of instructional content (i.e., learning). That is, the second hypothesis of this dissertation predicted that students participating in a Zoom lesson with an instructor who strategically uses Zoom features to enact the signaling, embodiment, and generative activity principles would experience less working memory overload and, in turn, exhibit greater learning compared to students in a Zoom lesson with an instructor who does not (see Figure 1).

H2: Compared to a standard synchronous lesson without Zoom features, students will 
experience less working memory overload and, in turn, exhibit greater learning in a synchronous lesson when their instructor uses Zoom features to enact the signaling, embodiment, and generative activity principles.

When examining the effects of instructors' implementation of CTML principles using specific Zoom features, it is important to consider alternative factors which could potentially influence student learning. Previous research specifically exploring the impact of CTML principles on learning (e.g., Clark et al., 2005; Mayer \& Pilegard, 2018; Pollock et al., 2002 Richter et al., 2016, 2018) have repeatedly identified prior knowledge of instructional content as a possible covariate (such that greater prior knowledge of instructional content inhibits the extent to which CTML principles enhance learning). Similarly, research exploring general predictors of student learning suggest that students' overall grade point averages (GPA) are also a significant predictor of learning outcomes (Richardson et al., 2012), as well as influence students' learning specifically during experimental studies (Bolkan \& Goodboy, 2019). Beyond prior knowledge and GPA, it is also plausible that different students may inherently find the subject matter of a given lesson or course particularly interesting, which may also influence the extent to which students learn material beyond a teacher's instructional design (Mazer, 2012). Given this, this dissertation also measured students' prior knowledge of lesson content, GPA, and interest as potential covariates which might influence student learning beyond an instructor's implementation of CTML principles via Zoom (see Figure 1). 
Figure 1

Hypothesized Conceptual Model of Hypothesis 2

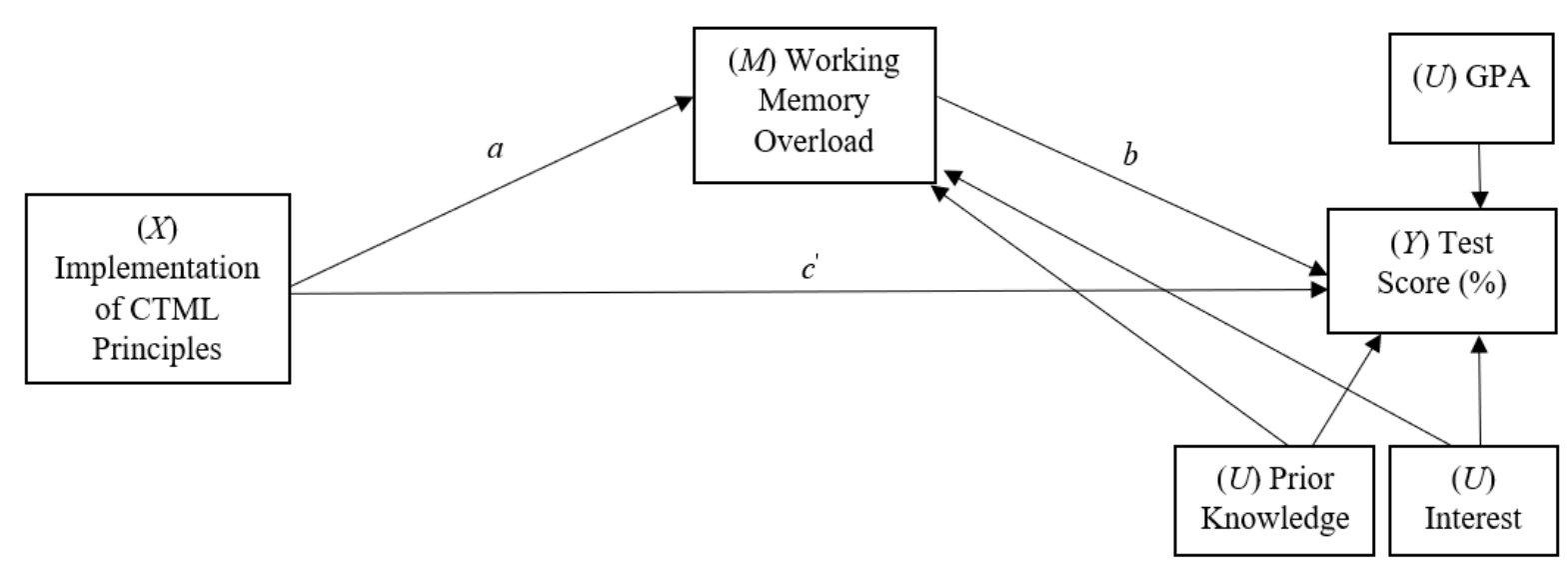

Note. Implementation of CTML Principles is indicator coded $(0=$ control, $1=$ treatment $)$ to reflect the mean difference between the two lesson conditions. Grade point average (GPA), prior knowledge related to lesson content (Prior Knowledge), and interest (Interest) are included in the model as covariates for student learning (Test Score).

Although the second hypothesis of this dissertation predicted that instructors can enhance students' learning by using Zoom features to reduce students' working memory overload, research has demonstrated that the extent to which students learn is not entirely dependent on instructors alone. As reviewed previously, CTML is based on the assumption that learning begins when individuals select specific pieces of novel information to pay attention to (Mayer, 2001). While CTML research has identified a variety of techniques that instructors can use to assist students in this regard (Mayer, 2001, 2005a, 2005b, 2005c, 2005d, 2014a, 2021), certain students may nevertheless possess an innate ability to better select relevant information and direct sustained attention toward that information than their peers. In particular, students' ability to select and focus on relevant information is fundamentally related to self-regulation, described as "proactive processes that students use to acquire academic skill, such as setting goals, 
selecting and deploying strategies, and self-monitoring one's effectiveness" (Zimmerman, 2008, p. 166). Students higher in self-regulation have a tendency to motivate themselves to engage with learning tasks, persist in scenarios where learning is difficult, and refrain from behaviors which might distract them from learning (Dabbagh \& Kitsantas, 2012). Further, self-regulated learners are better able to overcome impediments imposed by ineffective instruction, finding ways to succeed "even when presented with incomprehensible material, inadequate study conditions, and confusing instruction" (Lange et al., 2017, p. 66). Given this, it is likely that highly self-regulated students are better able to select relevant information to pay attention to during online lessons, even without an instructor's assistance. That is, an instructor's efforts to implement CTML principles into their online teaching may make less of a difference in reducing working memory overload for highly self-regulated students. Interestingly, Lang et al. (2017) suggested that the extent to which this is true may be limited, reporting that the positive association between selfregulation and learning was diminished when the lessons in which students participated entailed greater extraneous information. As such, this dissertation was also interested in exploring the extent to which instructors' implementation of CTML principles via Zoom might influence students' working memory overload and overall learning differently based upon the degree to which students are self-regulated (see Figure 2).

RQ1: To what extent does students' self-regulation moderate the influence of an instructor's implementation of the signaling, embodiment, and generative activity principles on students' working memory overload and, subsequently, learning? 
Figure 2

\section{Conceptual Model for Research Question 1}

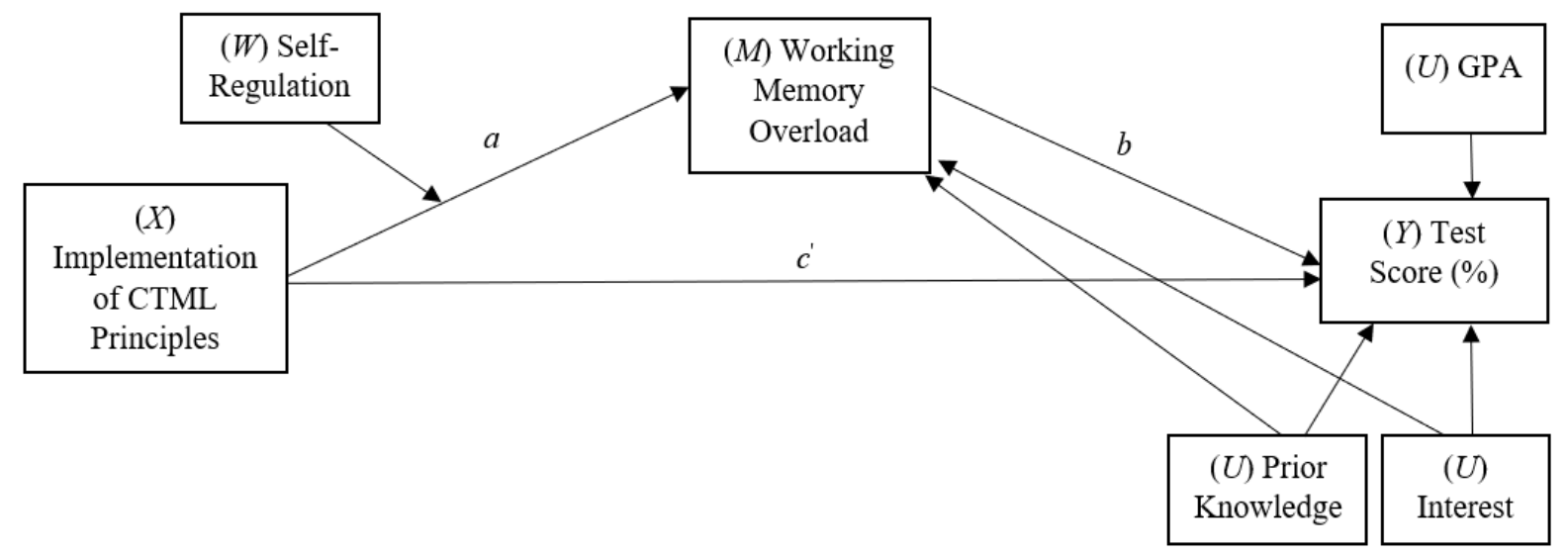

Note. Implementation of CTML Principles is indicator coded $(0=$ control, $1=$ treatment $)$ to reflect the mean difference between the two lesson conditions. Grade point average (GPA), prior knowledge related to lesson content (Prior Knowledge), and interest (Interest) are included in the model as covariates for student learning (Test Score).

The third hypothesis of this dissertation predicted that students participating in a synchronous lesson with an instructor who strategically uses Zoom features to enact CTML principles would also exhibit greater affect for their instructor compared to students in a lesson with an instructor who does not. Recall that the theoretical rationale for the embodiment principle is that students in multimedia lessons who can see their instructors engaging in humanlike body movements are more likely to positively identify with those instructors, thereby stimulating students' sense of social presence (Mayer, 2014a). As reviewed previously, there is ample empirical evidence to suggest this is the case, such that instructors' use of gestures (e.g., Li et al., 2019; Mayer \& DaPra, 2012; Wang et al., 2018) and eye contact (e.g., Fiorella et al., 2019, 2020; Stull et al., 2018) have been found to facilitate students' sense of social presence in a manner conducive to enhanced learning during multimedia lessons. Similarly, instructional 
communication research also suggests that instructors' nonverbal behaviors can significantly influence students' learning experiences, such that instructors who are nonverbally immediate enhance their students' motivation (Frymier, 1994; Frymier et al., 2019), participation (Rocca, 2009), and positive affective experiences (Witt et al., 2004). Given this, it is feasible that students participating in multimedia lessons where they can see an instructor engaging in nonverbally immediate behaviors will experience greater positive affect for that instructor than students in a lesson where they cannot. Further, studies suggest that clear teaching, in and of itself, may be positively related to students' affect for their instructors (see Titsworth et al., 2015), such that students experience greater positive affect for teachers who effectively assist them in selecting, understanding, and retaining material (Titsworth \& Mazer, 2016). Given this, it was anticipated that students in an online lesson with an instructor who uses Zoom features to enact CTML principles would report greater positive affective for their instructor than students in a lesson with an instructor who does not.

H3: Compared to a standard synchronous lesson without Zoom features, students will report greater positive affect for their instructor in a synchronous lesson where their instructor uses Zoom features to enact the signaling, embodiment, and generative activity principles. 


\section{Summary}

This chapter provided an overview of CTML (Mayer, 2001) - specifically, its underlying assumptions and CTML-based principles of effective instructional design in multimedia contexts - to explore potential strategies that instructors might use to enhance their online teaching via Zoom. Based on previous research exploring the impact of CTML-based principles of instructional design on student learning, this dissertation identified the signaling principle, embodiment principle, and generative activity principles of CTML as particularly applicable to Zoom teaching. This dissertation hypothesized that applying these three CTML-based principles of effective instructional design via strategic use of specific Zoom features would decrease the likelihood of students experiencing working memory overload and, in turn, enhance student learning. Further, this dissertation inquired the extent to which an instructor's implementation of CTML-based principles might have different effects for students with varying levels of selfregulation, as well as hypothesized that students would exhibit greater affect for instructors who implement CTML-based principles into their online teaching. 


\section{CHAPTER II}

\section{Methodology}

\section{Pilot Study}

\section{Participants and Procedures}

Prior to the main experiment, online lesson materials were pilot tested using 32 undergraduate students enrolled in an introduction to communication research methods course at West Virginia University. Participants' ages ranged from 18 to 23 years $(M=19.38, S D=1.39)$, with 12 participants identifying as male and 20 participants identifying as female when asked to report their sex. When asked to indicate their class rank, 14 participants reported that they were first-year students, 12 participants reported that they were sophomores, five participants reported that they were juniors, and one participant reported that they were a senior. Of the 32 participants, 30 participants identified as White/Caucasian and two participants identified as Black/African American.

Pilot study participants attended a live online lesson via Zoom in which the instructor (an early thirties White/Caucasian male dressed in business casual attire) taught from the script provided in Appendix A to enact the signaling, embodiment, and generative activity principles, using the PowerPoint presentation provided in Appendix B. The topic of the online lesson was college student development, based on Arthur Chickering's (1969) theory of college student development. The lesson was comprised of a brief introduction followed by an overview of the theory of college student development, a review of the seven vectors across which Chickering proposed college students develop their personal identities, an overview of the seven environmental factors which Chickering argued can influence college students' identity development, and a brief review. Content for the lesson was adapted from Evans et al. (2010). 
College student development was selected as a topic because it is a unique subject that is not addressed in undergraduate level courses in the Department of Communication Studies at West Virginia University, reducing the likelihood of participants in either the pilot study or main experiment having been exposed to information presented during the lesson previously.

\section{Instrumentation}

Upon completing the lesson, pilot study participants completed a post-lesson survey (see Appendix C). This survey was comprised of questions designed to measure the extent to which experimental manipulations of the signaling, embodiment, and generative activity principles were effective. Experimental manipulations of the signaling principle were assessed using an adapted version of Bolkan's (2018) Selection Scale, wherein the six survey items were revised to refer to the specific online lesson in which students participated (e.g., "I found it easy to identify the crucial aspects of this lesson"). Participant responses were solicited using a 7-point Likert scale ranging from 1 (Strongly Disagree) to 7 (Strongly Agree) $(M=5.84, S D=1.13)$.

The experimental manipulation of the embodiment principle was assessed by presenting participants with the question, "Was the instructor visible during the lesson you just participated in?" Participants responded to this question by selecting either "Yes" or "No." All pilot study participants who responded in the affirmative (100\% of pilot study participants) were subsequently presented with 18 items adapted from Richmond and colleagues' (2003) Nonverbal Immediacy Scale - Observer Report in order to determine the effectiveness with which the instructor enacted high-embodiment behaviors (i.e., moved in human-like ways). Items from this instrument were revised to be applicable to the online lesson that participants attended, such that 18 items were modified from the original instrument to refer to teaching behaviors in an online classroom (e.g., "He avoids eye contact while talking to people" was revised to "He avoids 
looking into the camera while talking to people") and eight items were removed from the original instrument given that they were not applicable to online instructional environments (e.g., "He touches others on the shoulder or arm while talking to them"). Participant responses were solicited using a 5-point Likert scale ranging from 1 (Strongly Disagree) to 5 (Strongly Agree) $(M=4.30, S D=.39)$, with higher scores indicating greater respondent perceptions of instructor immediacy.

Experimental manipulations of the generative activity principle were assessed using an adapted version of Bolkan's (2017) Organization Scale, such that the five survey items were revised to specifically refer to the Zoom polls used during the online lesson that participants attended (e.g., "The three polls helped me organize the material presented in this lesson in a logical manner"). Participant responses were solicited using a 7-point Likert scale ranging from 1 (Strongly Disagree) to 7 (Strongly Agree) $(M=5.76, S D=1.01)$.

The post-lesson survey completed by pilot study participants also included an adapted version of Cho and colleague's (2012) Plausibility Scale in order to assess participants' perceptions concerning the realism of the online lesson. This scale is comprised of five items, each of which were revised to apply to the online lesson that participants attended. Specifically, adapted items read as follows: "The instructor taught this online lesson in a way that could possibly happen in real life," "The online lesson taught by the instructor portrayed a possible real-life online lesson," "The online lesson could actually happen in real life," "Never in a real online classroom would someone teach the way that this instructor taught this online lesson," and "A real instructor would never teach an online lesson the way that this instructor did." Participant responses were solicited using a 7-point Likert scale ranging from 1 (Strongly Disagree) to 7 (Strongly Agree) $(M=6.21, S D=.90)$, with higher scores indicating greater perceived realism. 
Pilot study participants also completed a 10-item test assessing retention of lesson content. Test questions were designed to measure information retention by employing a series of multiple-choice questions assessing basic recall of lesson content (i.e., memorization). Each multiple-choice question was developed based on Suskie's (2018) guidebook for assessing student learning and employed four possible answers (a, b, c, d). Responses to multiple-choice questions were coded as (1) for correct responses and (0) for incorrect responses, then scored to reflect the percentage of total multiple choice questions answered correctly $(K R-20=.45, M=$ $64.38 \%, S D=17.40 \%$, Range $=30 \%-100 \%)$

\section{Results}

Although descriptive statistics suggested that experimental manipulations of the signaling, embodiment, and generative activity principles were effective and perceived as realistic by participants, pilot data highlighted potential issues regarding the 10 -item test designed to assess student learning. In addition to a low $K R-20(.45)$ and average score $(M=$ $64.38 \%$ ), descriptive statistics revealed that roughly $40 \%$ of participants received a failing grade on the test (60\% or lower). To address these issues, the researcher examined questions which pilot study participants answered incorrectly to identify issues in how questions were developed and revise accordingly. During this review, the researcher identified inconsistent language when comparing the lecture script to the wording of test questions which a majority of pilot study participants answered incorrectly, rewording those questions to ensure consistency with the specific language used by the instructor during the lesson. Similarly, in scenarios where a majority of pilot study participants selected the same incorrect answer when responding to a question, that response option was replaced with an appropriate substitute based on the content addressed by a given question. Using this method, five questions were revised - three by revising 
the wording of questions to ensure linguistic consistency with the lecture script and two by both revising the wording of questions and substituting problematic response options.

\section{Main Study}

\section{Participants}

Participants in the main study were 160 undergraduate students currently enrolled at West Virginia University and taking a course in the Department of Communication Studies (specifically courses listed in the department's IRB-approved departmental research policy). Participants' ages ranged from 18 to 54 years $(M=21.05, S D=5.23)$, with 60 participants identifying as male, 99 participants identifying as female, and one participant indicating that they "Prefer not to answer" when asked to report their sex. When asked to indicate their class rank, 59 participants reported that they were first-year students, 36 participants reported that they were sophomores, 31 participants reported that they were juniors, and 33 participants reported that they were seniors, with one participant reporting that their class rank was "Other." Of the 160 participants, 118 participants identified as White/Caucasian, 21 participants identified as Middle Eastern, eight participants identified as Black/African American, five participants identified as Asian/Asian American, three participants identified as Hispanic, and three participants identified as "Other" (e.g., "biracial," "mixed," and "mixed black and white") when asked to indicate which ethnicity they most closely identify with. Two respondents did not report their ethnicity. Participants' GPAs ranged from 1.90 to $4.00(M=3.30, S D=.55)$.

Of the 160 total participants recruited for this study, data provided by 18 were omitted from analyses due to participants having had previous experiences as students enrolled in courses taught by the instructor of the online lesson (the same early thirties White/Caucasian male dressed in business casual attire who taught the pilot study lesson). Data provided by an 
additional two participants were also omitted from analyses due to participants in the main study's second round of data collection indicating that they had already participated in previous data collection activities. Following the omission of these data, the final sample size for the main study of this dissertation was 140 participants. Of the final 140 participants, 72 participated in an online lesson in which the instructor used Zoom features to enact CTML principles (i.e., the treatment lesson) and 68 participated in a standard online lesson in which the instructor did not (i.e., the control lesson).

\section{Procedures}

To participate in this study, participants were required to meet the following inclusion criteria: be (1) a student at West Virginia University, (2) currently enrolled in a Communication Studies course listed in the IRB-approved departmental research policy, (3) at least 18 years of age or older, and (4) available to participate in an online lesson held on any of the potential dates and times identified in the announcements posted to the departmental bulletin board and departmental website (see Appendix D), in the online cover letter accompanying the sign-up survey (see Appendix E), or in the IRB-approved email announcement distributed by Communication Studies course instructors to interested students (see Appendix F). The researcher coordinated with instructors teaching Communication Studies courses listed in the IRB-approved departmental research policy and requested that instructors share email announcements with their students. Each announcement included a hyperlink directing potential participants to an online survey hosted via Qualtrics, through which potential participants were provided with instructions regarding how to sign-up to participate in this study. Per the IRBapproved departmental research policy, participants in this study were eligible to earn minimal extra credit in a Communication Studies course of their choosing (as long as it is listed in the 
department's IRB-approved departmental research policy) for their participation in this study.

Upon accessing the hyperlink referred to in the announcements posted to the departmental bulletin board and departmental website or in the IRB-approved email announcement distributed by Communication Studies course instructors to interested students, participants were directed to an online sign-up survey (see Appendix G). Upon accessing this sign-up survey, participants were presented with an online cover letter providing details related to this study. Participants were directed to exit the sign-up survey if they disagreed to the terms of the study as outlined in the online cover letter. Participants were required to indicate that they agreed to the terms of the study in order to sign-up to participate via the sign-up survey.

If participants agreed to the study's terms as outlined in the online cover letter of the sign-up survey, they were randomly assigned to attend one of the two online lessons and provided with (1) the date and time of their assigned lesson, (2) a hyperlink to access their assigned lesson, and (3) a password to access their assigned lesson. In addition, the sign-up survey prompted participants to create a unique alpha-numeric identification code to use in lieu of their names during their assigned online lesson. Participants created their identification code by providing the first three letters of the name of their hometown followed by the last four digits of their telephone number (e.g., NEW2651, MOR5473), and were prompted to (1) enter their identification code on the sign-up survey to complete their registration for the study and (2) record the identification code for their personal records. As articulated in the recruitment scripts and online consent form accompanying the sign-up survey, participants were only eligible to attend the specific online lesson to which they were assigned. Participants were not provided with hyperlinks or passwords to access alternative online lessons, and participants who did not provide an identification code registered for the online lesson they attended became ineligible to 
receive extra credit for participating in this study.

At the date and time of their assigned online lesson, participants accessed the online lesson using the hyperlink and password provided they were provided with when completing the sign-up survey. Identical content was taught in both online lessons, and the same PowerPoint slides (see Appendix H) were used in identical ways during each lesson. The only differences between the two online lessons was whether the instructor utilized Zoom features to enact CTML principles of instructional design during the lesson.

The hyperlink provided to participants assigned to the standard online lesson in which the instructor did not enact CTML principles (i.e., the control condition) directed them to a Zoom meeting room. Participants accessed the lesson using the password they were provided upon signing up for this study and entered their unique identification code in lieu of their names, as directed during the sign-up survey. At the date and time specified in the materials provided to participants upon being randomly assigned to this online lesson when completing the sign-up survey, the lesson was locked to prevent subsequent attendees from joining and the instructor began teaching the lesson using the script provided in Appendix I. Throughout the lesson, participants viewed a PowerPoint presentation of lesson information accompanied by instructor narration. The instructor was not visible to participants in this lesson. All participants in this online lesson had their microphones muted, were unable to activate their cameras or display images, and were unable to type or send messages using Zoom chat. Upon completing the lesson, the instructor thanked participants for their time and provided them with a hyperlink to a unique online Qualtrics survey using Zoom chat. Due to challenges in recruiting participants for this study, data collection activities took place twice, requiring the instructor to teach this lesson twice. The first control lesson taught by the instructor lasted 20:31 and the second lasted 21:15. 
The hyperlink provided to participants assigned to the CTML-based online lesson (i.e., the treatment condition) directed them to a Zoom meeting room. Participants accessed the lesson using the password they were provided upon signing up for this study and entered their unique identification code in lieu of their names, as directed during the sign-up survey. At the date and time specified in the materials provided to participants upon being randomly assigned to this online lesson when completing the sign-up survey, the lesson was locked to prevent subsequent attendees from joining and the instructor began teaching the lesson using the script provided in Appendix J. Throughout the lesson, participants viewed a PowerPoint presentation of lesson information accompanied by instructor narration, as well as by a live video of the instructor in a small box on the right side of their screen. Participants were able to see the instructor teaching for the duration of the lesson. The instructor was an early-thirties man dressed in business-casual attire, and was the same instructor who taught the online lesson for participants assigned to the control condition. All participants in this online lesson condition had their microphones muted, were unable to activate their cameras or display images, and were unable to type or send messages using Zoom chat. Upon completing the lesson, the instructor thanked participants for their time and provided them with a hyperlink to a unique online Qualtrics survey using Zoom chat. Due to challenges in recruiting participants for this study, data collection activities took place twice, requiring the instructor to teach this lesson twice (similar to the control lesson). The first treatment lesson taught by the instructor lasted 28:08, and the second lasted 28:10.

Upon completing the post-lesson survey, which assessed working memory overload, selection and organization of lesson content, learning, interest in lesson content, prior knowledge, GPA, and demographic information, participants were provided with an additional hyperlink to a separate research receipt survey. A unique research receipt survey was provided to 
participants in each condition. The research receipt survey was only accessible to participants who activated its corresponding hyperlink from the URL of the final page on the appropriate post-lesson survey for the experimental lesson they attended. The research receipt survey prompted participants to provide their name, West Virginia University email address, the unique alphanumeric identification code they created and used throughout the study, and the course and instructor for which they were seeking extra credit. The unique identification number which participants provided was cross-referenced with the identification codes registered for the specific experimental lesson that participants sought extra credit for participating in. Extra credit information provided by participants who failed to provide an identification code registered for the appropriate experimental condition were removed. Once the researcher completed crossreferencing identification codes, the identification codes were removed from the data for each research receipt survey dataset to ensure that individual participants could no longer be paired with data provided in the post-lesson survey. Research receipt data was then aggregated, sorted by courses in which participants were seeking extra credit, separated into course-specific datasets, and shared with the appropriate instructors in the Department of Communication Studies.

\section{Instrumentation}

Similar to the pilot study, the post-lesson survey was comprised of questions designed to measure the extent to which participants were able to effectively select lesson content to pay attention to, see their instructor, and organize instructional material. Participants' ability to select information was assessed using an adapted version of Bolkan's (2018) Selection Scale (see Appendix K), wherein the six survey items were revised to refer to the specific online lesson in which students participated (e.g., "I found it easy to identify the crucial aspects of this lesson"). 
Participant responses were solicited using a 7-point Likert scale ranging from 1 (Strongly

Disagree) to 7 (Strongly Agree) in both the treatment $(\omega=.95$ [95\% CI: .91, .97], $M=6.10, S D=$ $1.07)$ and control $(\omega=.96$ [95\% CI: .91, .98], $M=5.89, S D=1.24)$ groups.

Participants' ability to see their instructor was assessed by presenting participants with the question, "Was the instructor visible during the lesson you just participated in?" Participants responded to this question by selecting either "Yes" or "No." All participants in the treatment group who responded to this question did so in the affirmative, and all participants in the control group who responded to this question did so in the negative.

Participants' ability to organize lesson content was assessed using an adapted version of Bolkan's (2017) Organization Scale (see Appendix L), such that the five survey items were revised to specifically refer to the online lesson that participants attended (e.g., "I was able to organize the material presented in this lesson in a logical manner”). Participant responses were solicited using a 7-point Likert scale ranging from 1 (Strongly Disagree) to 7 (Strongly Agree) for participants assigned to both the treatment $(\omega=.92$ [95\% CI: .87, .95], $M=6.33, S D=.80)$ and control $(\omega=.91[95 \% \mathrm{CI}: .84, .96], M=6.18, S D=.82)$ conditions.

Participants' working memory overload was measured using the working memory overload subscale from Bolkan's (2017a) Clarity Indicators Scale (see Appendix M). This instrument utilizes a seven-point Likert response format ranging from (1) strongly disagree to (7) strongly agree, presenting respondents with statements such as "The amount of information presented in the lesson was overwhelming," and "I felt flustered trying to keep up with the amount of information presented in this lesson" (treatment: $\omega=.95$ [95\% CI: .90, .98], $M=2.19$, $S D=1.46$; control: $\omega=.95$ [95\% CI: .91, .97], $M=2.46, S D=1.46$ ).

Participants' learning was measured using a revised iteration of the 10-item test assessing 
retention of lesson content which pilot study participants completed (see Appendix N). Test questions were designed to measure information retention by employing a series of multiplechoice questions assessing basic recall of lesson content (i.e., memorization). Each multiplechoice question was developed based on Suskie's (2018) guidebook for assessing student learning and employed four possible answers (a, b, c, d), as well as modified based on pilot study data such that questions utilized language consistent with the lecture scripts and problematic response options were removed. Responses to multiple-choice questions were coded as (1) for correct responses and (0) for incorrect responses, then scored to reflect the percentage of total multiple choice questions answered correctly. The percentage mean, standard deviation, range, KR-20 reliability value, and the number of participants who received each test score are provided in Table 1. The percentages for each test question answered correctly and incorrectly are provided in Table 2 . 
Table 1

\begin{tabular}{|c|c|c|c|c|c|c|c|c|c|c|c|c|c|c|}
\hline \multicolumn{15}{|c|}{ Descriptive information for the 10-item test } \\
\hline Condition & $M$ & $S D$ & $K R-20$ & Range & $10 \%$ & $20 \%$ & $30 \%$ & $40 \%$ & $50 \%$ & $60 \%$ & $70 \%$ & $80 \%$ & $90 \%$ & $100 \%$ \\
\hline $\begin{array}{l}\text { Treatment } \\
\text { Condition } \\
(n=72)\end{array}$ & 69.86 & 19.25 & .55 & $20-100 \%$ & 0 & 1 & 2 & 6 & 7 & 12 & 12 & 12 & 16 & 4 \\
\hline $\begin{array}{l}\text { Control } \\
\text { Condition } \\
(n=68)\end{array}$ & 61.32 & 22.39 & .63 & $10-100 \%$ & 2 & 1 & 5 & 9 & 11 & 9 & 10 & 11 & 5 & 5 \\
\hline
\end{tabular}

Note. The rightmost ten columns present the number of participants who received each test score (e.g., $80 \%$ indicates that a respondent answered eight of the total ten test questions correctly).

Table 2

\begin{tabular}{|c|c|c|c|c|c|c|c|c|c|c|}
\hline \multicolumn{11}{|c|}{ Percentages of correct and incorrect answers for the 10-item test } \\
\hline $\begin{array}{l}\text { Condition } \\
\text { Treatment } \\
\text { Condition } \\
(n=72)\end{array}$ & Q1 & Q2 & Q3 & $\mathrm{Q}^{4}$ & Q5 & Q6 & Q7 & Q8 & Q9 & Q10 \\
\hline Correct & $95.8 \%$ & $81.9 \%$ & $36.1 \%$ & $58.3 \%$ & $61.1 \%$ & $68.1 \%$ & $80.6 \%$ & $88.9 \%$ & $62.5 \%$ & $65.3 \%$ \\
\hline $\begin{array}{l}\text { Incorrect } \\
\text { Control } \\
\text { Condition } \\
(n=68)\end{array}$ & $4.2 \%$ & $18.1 \%$ & $63.9 \%$ & $41.7 \%$ & $38.9 \%$ & $31.9 \%$ & $19.4 \%$ & $11.1 \%$ & $37.5 \%$ & $34.7 \%$ \\
\hline Correct & $88.2 \%$ & $75.0 \%$ & $30.9 \%$ & $48.5 \%$ & $48.5 \%$ & $58.8 \%$ & $73.5 \%$ & $64.7 \%$ & $66.2 \%$ & $58.8 \%$ \\
\hline Incorrect & $11.8 \%$ & $25.0 \%$ & $69.1 \%$ & $51.5 \%$ & $51.5 \%$ & $41.2 \%$ & $26.5 \%$ & $35.3 \%$ & $33.8 \%$ & $41.2 \%$ \\
\hline
\end{tabular}


Participants' affect toward the instructor of the online lesson they attended was measured using two subscales from McCroskey's (1994) Instructional Affect Assessment Instrument (see Appendix O). The first subscale presents respondents with the stem "My attitude about this instructor is:", followed by four 7-point semantic differential scales ranging from (1) good to bad, (2) valuable to worthless, (3) fair to unfair, and (4) positive to negative (treatment: $\omega=.66$ [95\% CI: .38, .86], $M=6.74, S D=.48$; control: $\omega=.67$ [95\% CI: .43, .84], $M=6.42, S D=.80)$. The second subscale presents respondents with the stem "The likelihood of actually enrolling in another course with this instructor if my schedule would permit would be:”, followed by four 7point semantic differential scales ranging from (1) likely to unlikely, (2) possible to impossible, (3) probable to improbable, and (4) would to would not (treatment: $\omega=.91$ [95\% CI: .85, .96], $M$ $=6.41, S D=.90 ;$ control: $\omega=.91$ [95\% CI: .81, .96], $M=5.99, S D=1.23$ ).

Participants' self-regulation was measured using four items taken from the resource management strategy subscale of the Motivated Strategies for Learning Questionnaire (Pintrich et al., 1991; see Appendix P). This scale assesses students' ability to control their attention and effort when faced with distractions or uninteresting tasks via a series of four statements to which participants respond on a scale from (1) not at all true of me to (7) very true of me. Given that the items included in this subscale refer to a particular class in which a student is enrolled, they were modified so that each item instead referred to students' self-regulation as a general tendency. Examples of items include "Even when materials are dull and uninteresting, I manage to keep working until I finish," and "When course work is difficult, I give up or only study the easy parts" (treatment: $\omega=.78$ [95\% CI: .60, .87], $M=5.36, S D=1.32$; control: $\omega=.72$ [95\% CI: .49, $.84], M=5.03, S D=1.21)$.

Prior knowledge, one of the three covariates included in this study, was assessed using 
the three-item Perceived Familiarity Scale developed by Bolkan and colleagues (2016; see Appendix Q). This instrument employs a 5-point Likert-type format with responses ranging from (1) not at all to (5) very much, which respondents use to respond to the questions "How familiar were you with the topic of this lesson before today," "How much did you already know about the topic of this lesson before today," and "To what extent had you been exposed to the material in this lesson in the past" (treatment: $\omega=.92[95 \%$ CI: .87, .95], $M=2.16, S D=.99$; control: $\omega=.91$ [95\% CI: .84, .95], $M=2.16, S D=1.06)$. GPA, the second covariate in this study, was measured by asking participants to (1) indicate their current GPA (treatment: $M=3.31, S D=.58$; control $M=3.30, S D=.53$ ) and (2) indicate their level of confidence regarding the accuracy of the GPA they provide on a scale from (1) not at all to (5) very (see Appendix R; treatment: $M=4.08, S D$ = 1.22; control: $M=4.21, S D=1.13$ ).

Interest in lesson material, the third covariate in this study, was measured using an adapted version of Mazer's (2012) Student Interest Scale (see Appendix S). This instrument is comprised of two subscales; one subscale comprised of nine items designed to assess students' emotional interest in course content (e.g., "The topics covered in the course fascinate me;" treatment: $\omega=.93$ [95\% CI: .90, .95], $M=3.84, S D=.70$; control: $\omega=.93$ [95\% CI: .87, .95], $M$ $=3.60, S D=.67)$ and a second subscale comprised of seven items designed to assess students' cognitive interest in course content (e.g., "The information covered in the course is making me more knowledgeable;" treatment: $\omega=.90$ [95\% CI: .85, .94], $M=4.05, S D=.62$; control: $\omega=.83$ [95\% CI: .73, .90], $M=3.92, S D=.54)$. Both subscales of this instrument were modified so that items referred to the online lesson which students participated in rather than a class in which they are enrolled (e.g., "The topics covered in the course fascinate me" was revised to "The topics covered in the lesson fascinated me"). 


\section{Data Analysis}

Hypothesis Testing. Hypothesis one, which predicted that participants in a CTML-based Zoom lesson would experience less working memory overload than participants in a Zoom lesson not based on CTML, was tested using a one-way Analysis of Variance (ANOVA) in SPSS Version 26. The two conditions were dummy coded as 0 (control) and 1 (treatment). Prior to conducting the ANOVA, the researcher tested underlying statistical assumptions of normality (via Kolmogorov-Smirnov and Shapiro-Wilk tests) and homogeneity of variance (via the Levene statistic). After conducting the one-way ANOVA, the research conducted a one-way Analysis of Covariance (ANCOVA) including familiarity with lesson content, GPA, and student interest as covariates.

Hypothesis two was tested using ordinary least squares path-analysis in Hayes' (2018) PROCESS version 3.5 macro for SPSS Version 26. Specifically, experimental conditions were dummy coded $($ control $=0$, treatment $=1)$, allowing the researcher to examine the inferred causal process through which an instructor's implementation of CTML principles during an online lesson $(X)$ influenced students' test performance $(Y)$ through working memory overload $(M)$, controlling for prior knowledge, GPA, and student interest (as shown in Figure 1). Relative indirect effects were estimated using 95\% confidence intervals based on 10,000 percentile bootstrap samples with replacement, and mediation effect sizes were interpreted using partially standardized relative indirect $\left(a b_{p s}\right)$ and direct $\left(c^{\prime}{ }_{p s}\right)$ effects (Hayes, 2018).

Research question one posed a question of moderated mediation, inquiring the extent to which students' self-regulation moderated the influence of an instructor's implementation of CTML principles on students' working memory overload and, subsequently, learning. This research question was examined via a conditional process model using Hayes' (2018) PROCESS 
version 3.5 macro for SPSS Version 26, with self-regulation entered as a first-stage moderator for the $a$ path (see Figure 2). Again, experimental conditions were dummy coded (control = 0, treatment $=1$ ). Similar to hypothesis two, relative indirect effects were estimated using $95 \%$ confidence intervals based on 10,000 percentile bootstrap samples with replacement. Whether the indirect effect of CTML-based teaching on students' test scores through working memory overload is conditional upon students' self-regulation was assessed via an examination of the index of moderated mediation (Hayes, 2015). Hayes (2015) wrote that "if the confidence interval [for the index of moderated mediation] does not include zero, this leads to the inference that the relationship between the indirect effect and the moderator is not zero - moderated mediation" ( $p$. 8). When the confidence interval for the index of moderated mediation does not include zero, "any two conditional indirect effects defined by different values of the moderator are statistically significant" (Hayes, 2015, p. 14). When moderated mediation is observed, it is appropriate to probe the moderation in order to explore the nature of conditional indirect effects, estimating conditional indirect effects at three values of the moderator $\left(16^{\text {th }}, 50^{\text {th }}\right.$, and $84^{\text {th }}$ percentiles $)$ and examining the $95 \%$ confidence intervals associated with each. Bootstrap confidence intervals which do not include zero are interpreted as "evidence of mediation at that value of [the moderator]" (Hayes, 2018, p. 493).

Hypothesis three, which predicted that participants in a CTML-based Zoom lesson would report greater affect for their instructor than participants in a Zoom lesson not based on CTML, was tested using a one-way ANOVA in SPSS Version 26. The two conditions were dummy coded as 0 (control) and 1 (treatment). Prior to conducting the ANOVA, the researcher tested underlying statistical assumptions of normality (via Kolmogorov-Smirnov and Shapiro-Wilk tests) and homogeneity of variance (via the Levene statistic). After conducting the one-way 
ANOVA, the research conducted a one-way Analysis of Covariance (ANCOVA) including familiarity with lesson content, GPA, and student interest as covariates. 


\section{Summary}

This chapter described the methodological procedures that were used in this dissertation. In particular, this chapter summarized the procedures which were used to pilot test experimental materials, reviewed the processes through which participants were recruited, outlined the procedures which were used to randomly assign participants across experimental conditions, and demonstrated the ways in which CTML principles of instructional design were enacted (or not enacted) in each experimental condition. Additionally, this chapter provided a description of the instruments which were used to measure variables of interest and identified the analyses which were used to explore the ways in which those variables related to one another. 


\section{CHAPTER III}

\section{Results}

Intercorrelations among variables are presented in Table 3, as well as composite means, standard deviations, and omega $(\omega)$ reliability coefficients with $95 \%$ confidence intervals from 10,000 bootstrap samples, calculated using Hayes and Coutts' (2020) OMEGA macro for SPSS.

\section{Hypothesis Testing}

\section{Hypothesis One}

Hypothesis one predicted that participants in a CTML-based Zoom lesson would experience less working memory overload than participants assigned to a Zoom lesson that was not based on CTML principles of instructional design. Prior to testing this hypothesis using a one-way ANOVA, the researcher tested underlying statistical assumptions of normality and homogeneity of variance prerequisite to conducting a one-way ANOVA. Both a KolmogorovSmirnov test $(p<.001)$ and a Shapiro-Wilk test $(p<.001)$ were significant, which prompted the researcher to assess the skewness and kurtosis of participants' scores in working memory overload. Although the $z$ score for kurtosis $(z=1.44)$ fell below the $|1.96|$ cutoff value for statistical significance, the $z$ score for skewness $(z=2.63)$ fell well beyond it. Based upon this evidence, normality of distribution was not assumed for participants' scores in working memory overload. In contrast, a Levene's test yielded a nonsignificant value $(p=.910)$, suggesting that the assumption for homogeneity of variance was met. 
Table 3

Intercorrelations among variables

\begin{tabular}{|c|c|c|c|c|c|c|c|c|c|c|c|c|c|c|}
\hline Variables & $M$ & $S D$ & $\begin{array}{c}\omega \\
{[\mathrm{LL}, \mathrm{UL}]}\end{array}$ & 1 & 2 & 3 & 4 & 5 & 6 & 7 & 8 & 9 & 10 & 11 \\
\hline 1. Selection & 6.00 & 1.15 & $\begin{array}{c}.95 \\
{[.92, .97]}\end{array}$ & -- & & & & & & & & & & \\
\hline 2. Organization & 6.26 & .81 & $\begin{array}{c}.91 \\
{[.88, .94]}\end{array}$ & $.57^{\wedge}$ & -- & & & & & & & & & \\
\hline 3. Working Memory Overload & 2.32 & 1.46 & $\begin{array}{c}.95 \\
{[.92, .97]}\end{array}$ & $-.36^{\wedge}$ & $-.44^{\wedge}$ & -- & & & & & & & & \\
\hline 4. Test Percentage & 65.71 & 21.19 & -- & .13 & $.17 *$ & $-.20 *$ & -- & & & & & & & \\
\hline 5. Affect Toward Instructor & 6.59 & .67 & $\begin{array}{c}.68 \\
{[.52, .81]}\end{array}$ & $.41^{\wedge}$ & $.45^{\wedge}$ & $-.36^{\wedge}$ & $.22 * *$ & -- & & & & & & \\
\hline 6. Affect Enroll with Instructor & 6.21 & 1.09 & $\begin{array}{c}.91 \\
{[.85, .95]}\end{array}$ & $.48^{\wedge}$ & $.52^{\wedge}$ & $-.33^{\wedge}$ & .11 & $.47^{\wedge}$ & -- & & & & & \\
\hline 7. Self-Regulation & 5.20 & 1.28 & $\begin{array}{c}.76 \\
{[.63, .83]}\end{array}$ & $.27 * *$ & $.37^{\wedge}$ & $-.26 * *$ & $.19 *$ & .14 & $.33^{\wedge}$ & -- & & & & \\
\hline 8. Familiarity & 2.16 & 1.02 & $\begin{array}{c}.91 \\
{[.87, .94]}\end{array}$ & .04 & .01 & -.12 & -.16 & -.09 & .07 & -.05 & -- & & & \\
\hline 9. GPA & 3.30 & .55 & -- & -.04 & .05 & .07 & .10 & .02 & -.04 & $.23^{\wedge}$ & -.11 & -- & & \\
\hline 10. Emotional Interest & 3.73 & .70 & $\begin{array}{c}.93 \\
{[.90, .95]}\end{array}$ & $.48^{\wedge}$ & $.51^{\wedge}$ & $-.27 * *$ & -.00 & $.32^{\wedge}$ & $.47^{\wedge}$ & $.34^{\wedge}$ & .11 & -.06 & -- & \\
\hline 11. Cognitive Interest & 3.98 & .59 & $\begin{array}{c}.88 \\
{[.83, .91]}\end{array}$ & $.52^{\wedge}$ & $.54^{\wedge}$ & $-.40^{\wedge}$ & .05 & $.29^{\wedge}$ & $.41^{\wedge}$ & $.35^{\wedge}$ & $.19 *$ & -.08 & $.74^{\wedge}$ & -- \\
\hline
\end{tabular}

Note. ${ }^{*} p<.05 . * * p<.01 .{ }^{\wedge} p<.001$. 
Results of the one-way ANOVA revealed no significant differences in participants' working memory overload between experimental conditions, Welch's $F(1,136)=1.196, p=$ $.276, \eta^{2}=.009$, suggesting that participants who attended the CTML-based Zoom lesson $(M=$ $2.19, S D=1.46$ ) did not report having experienced significantly different working memory overload than participants who attended the Zoom lesson which was not designed based upon CTML principles $(M=2.46, S D=1.46)$. Given this, hypothesis one was not supported. A subsequent ANCOVA, controlling for participants' interest in lesson content, familiarity with lesson content, and GPA, similarly failed to detect significant differences between levels of working memory overload reported by participants who attended the CTML-based Zoom lesson $\left(M_{\text {adj }}=2.47\right)$ and the Zoom lesson which was not designed based upon CTML principles $\left(M_{\text {adj }}=\right.$ $2.25), F(1,121)=.800, p=.373, \eta_{\mathrm{p}}^{2}=.007$.

\section{Hypothesis Two}

Hypothesis two predicted that participants who attended a CTML-based Zoom lesson would experience less working memory overload and, in turn, exhibit greater learning on a postlesson test than participants who attended a Zoom lesson which was not based on CTML principles of instructional design. The researcher tested this hypothesis using a simple mediation model (Model 4; Hayes, 2018), estimating indirect effects using 95\% confidence intervals based on 10,000 percentile bootstrap samples. The mediation analysis failed to uncover an indirect effect for the influence of CTML-based instruction on participants' test performance through working memory overload $\left(a b=.703, \mathrm{CI}:-.530,2.520 ; a b_{p s}=.034, \mathrm{CI}:-.026, .122\right)$. In contrast, the analysis revealed a direct effect for the influence of CTML-based principles on participants' test performance $\left(c^{\prime}=7.367, c_{p s}^{\prime}=.350, \mathrm{CI}: .470,14.265\right)$, such that the implementation of CTML-based principles of instructional design via Zoom features directly caused an average 
increase of seven percent across participants' test scores, holding constant working memory

overload. Given this, although CTML-based teaching did lead to increases in students' learning as evidenced by the post-lesson test, hypothesis two was not supported. Unstandardized model estimates for the analysis are provided in Table 4.

Table 4

Unstandardized model estimates for hypothesis 2

\begin{tabular}{|c|c|c|c|c|c|c|}
\hline H2 - CTML Principles & \multicolumn{6}{|c|}{ Consequent } \\
\hline \multirow{2}{*}{$\begin{array}{l}a b=.703, \mathrm{CI}:-.530,2.520 \\
c^{\prime}=7.367, p=.037\end{array}$} & \multicolumn{3}{|c|}{$F(1,137)=1.196, p=.276, R^{2}=.01$} & \multicolumn{3}{|c|}{$F(2,136)=5.045, p=.008, R^{2}=.07$} \\
\hline & \multicolumn{3}{|c|}{ Working Memory Overload } & \multicolumn{3}{|c|}{ Test Percentage } \\
\hline Antecedent & Estimate & $S E$ & $p$ & Estimate & $S E$ & $p$ \\
\hline CTML Lesson Condition & -.272 & .248 & .276 & 7.367 & 3.488 & .037 \\
\hline Working Memory Overload & -- & -- & -- & -2.588 & 1.195 & .032 \\
\hline
\end{tabular}

Note. Estimates highlighted in bold text indicate significant unstandardized regression slopes.

The researcher also tested the mediation model while controlling for students' interest in lesson content, familiarity with lesson content, and GPA as potential covariates. The researcher conducted this analysis using the same simple mediation model (Model 4; Hayes, 2018) as when testing hypothesis two, but also included four covariates (emotional interest, cognitive interest, familiarity, and GPA). This mediation analysis failed to uncover any indirect effect for the influence of CTML-based instruction on participants' test performance through working memory overload $\left(a b=.697, \mathrm{CI}:-.847,2.651, a b_{p s}=.034, \mathrm{CI}:-.041, .130\right)$, as well as failed to reveal a direct effect for the influence of CTML-based principles on participants' test performance $\left(c^{\prime}=\right.$ 5.283, $\left.c_{p s}^{\prime}=.260, \mathrm{CI}:-1.725,12.291\right)$. Unstandardized model estimates, including covariates, are provided in Table 5. 
Table 5

Unstandardized model estimates (including covariates) for hypothesis 2

\begin{tabular}{|c|c|c|c|c|c|c|}
\hline \multirow{3}{*}{$\begin{array}{l}\mathrm{H} 2-\mathrm{CTML} \text { Principles } \\
a b=.697, \mathrm{CI}:-.847,2.651 \\
c^{\prime}=5.283, p=.138\end{array}$} & \multicolumn{6}{|c|}{$\underline{\text { Consequent }}$} \\
\hline & \multicolumn{3}{|c|}{$F(5,121)=4.06, p=.002, R^{2}=.14$} & \multicolumn{3}{|c|}{$F(6,120)=3.05, p=.008, R^{2}=.13$} \\
\hline & \multicolumn{3}{|c|}{ Working Memory Overload } & \multicolumn{3}{|c|}{ Test Percentage } \\
\hline Antecedent & Estimate & $S E$ & $p$ & Estimate & $S E$ & $p$ \\
\hline CTML Lesson Condition & -.219 & .245 & .373 & 5.283 & 3.539 & .138 \\
\hline Working Memory Overload & -- & -- & -- & -3.178 & 1.307 & .017 \\
\hline Familiarity & -.111 & .127 & .379 & -4.670 & 1.827 & .012 \\
\hline Emotional Interest & .063 & .258 & .808 & -3.512 & 3.708 & .345 \\
\hline Cognitive Interest & -.879 & .309 & .005 & 4.819 & 4.590 & .296 \\
\hline GPA & -- & -- & -- & 3.275 & 3.134 & .293 \\
\hline
\end{tabular}

Note. Estimates highlighted in bold text indicate significant unstandardized regression slopes.

\section{Research Question One}

Research question one inquired if participants' self-regulation might moderate the influence of an instructor's implementation of CTML principles on students' working memory overload and, subsequently, learning. That is, research question one was concerned with moderated mediation. Using PROCESS 3.5 (Hayes, 2018), the researcher tested the conditional process model with students' self-regulation serving as a first stage moderator. Ordinary least squares path coefficients for the moderated mediation are presented in Table 6. Moderated mediation was assessed using Hayes' (2015) index of moderated mediation, and all analyses in the model used 10,000 bootstrap samples and percentile bootstrap confidence intervals. The index of moderated mediation provided no evidence to suggest that participants' self-regulation moderated an indirect effect for CTML-based instruction on test performance through working memory overload (index of moderated mediation $=.055$, bootstrap CI: $-1.225,1.047)$. Given that moderated mediation was not observed, the researcher refrained from probing conditional indirect effects. 
Table 6

OLS path model coefficients: First stage moderated by self-regulation

$\underline{\text { Models }}$

$\begin{array}{lll}\text { B } & \underline{\text { SE }} \quad \underline{\text { LLCI }} \quad \underline{\text { ULCI }}\end{array}$

Working Memory Overload

$F(3,133)=3.229, p=.025, R^{2}=.068$

Constant

$\begin{array}{llll}3.832 & .759 & 2.331 & 5.333\end{array}$

CTML-Based Instruction $(a)$

$\begin{array}{llll}-.048 & 1.043 & -2.111 & 2.015\end{array}$

Self-Regulation

$\begin{array}{llll}-.274 & .147 & -.564 & .017\end{array}$

CTML-Based Instruction*Self-Regulation

$\begin{array}{llll}-.021 & .026 & -.409 & .366\end{array}$

$F(2,134)=4.922, p=.009, R^{2}=.068$

Constant

Test Performance (\%)

Working Memory Overload (b)

$\begin{array}{llll}68.199 & 3.901 & 60.484 & 75.914\end{array}$

CTML-Based Instruction (Relative Direct Effect; $c$ )

$\begin{array}{llll}-2.600 & 1.207 & -4.987 & -.212\end{array}$

$\begin{array}{llll}7.390 & 3.537 & .395 & 14.385\end{array}$

Note. Standard errors and confidence intervals are generated using percentile bootstrapping from 10,000 samples.

The researcher also tested a conditional process model including familiarity with lesson content, interest in lesson content, and GPA as potential covariates, once again entering participants' self-regulation as a first stage moderator. Ordinary least squares path coefficients for the moderated mediation are presented in Table 7. Moderated mediation was assessed using Hayes' (2015) index of moderated mediation, and all analyses in the model used 10,000 bootstrap samples and percentile bootstrap confidence intervals. Once again, the index of moderated mediation provided no evidence to suggest that participants'self-regulation moderated an indirect effect for CTML-based instruction on test performance through working memory overload (index of moderated mediation $=.318$, bootstrap CI: $-.938,1.449$ ). Given that moderated mediation was not observed, the researcher refrained from probing conditional indirect effects. 
Table 7

OLS path model coefficients: First stage moderated by self-regulation (including covariates)

Models

$\begin{array}{lll} & \text { Bootstrapped } \\ \quad & \underline{\text { SE }} \quad \underline{\text { LLCI }} \quad \underline{\text { ULCI }}\end{array}$

Working Memory Overload

$F(7,118)=3.085, p=.005, R^{2}=.155$

Constant

$\begin{array}{llll}5.427 & 1.321 & 2.811 & 8.042\end{array}$

CTML-Based Instruction $(a)$

$\begin{array}{llll}.337 & 1.059 & -1.759 & 2.433\end{array}$

Self-Regulation

$\begin{array}{llll}-.063 & .062 & -.384 & .259\end{array}$

CTML-Based Instruction*Self-Regulation

$-.101$

.202

$-.501 \quad .299$

Familiarity

$-.126$

$\begin{array}{lll}.129 & -.382 & .130\end{array}$

Emotional Interest

.088

$\begin{array}{lll}.261 & -.429 & .604\end{array}$

Cognitive Interest

$\begin{array}{llll}-.817 & .319 & -1.448 & -.185\end{array}$

$F(6,119)=3.059, p=.008, R^{2}=.134$

Constant Test Performance (\%)

Working Memory Overload (b)

63.001

18.353

$\begin{array}{rr}26.661 & 99.342 \\ -5.750 & -.554 \\ -2.006 & 12.142 \\ -8.437 & -1.132 \\ -10.915 & 3.814 \\ -4.088 & 14.241 \\ -2.845 & 9.635\end{array}$

Note. Standard errors and confidence intervals are generated using percentile bootstrapping from 10,000 samples.

\section{Hypothesis Three}

Hypothesis three predicted that participants who attended the CTML-based Zoom lesson would report greater affect for their instructor than participants assigned to the Zoom lesson in which their instructor refrained from implementing CTML-based best practices. Prior to testing this hypothesis using one-way ANOVAs for participants' ratings of affect toward their instructor and affect toward enrolling in future courses with their instructor, the researcher tested underlying statistical assumptions of normality and homogeneity of variance. Both a Kolmogorov-Smirnov test $(p<.001)$ and a Shapiro-Wilk test $(p<.001)$ were significant for participants' ratings of affect toward the instructor, prompting the researcher to assess their skewness and kurtosis values. Both the $z$ score for kurtosis $(z=4.76)$ and the $z$ score for 
skewness $(z=-4.12)$ fell well beyond the $|1.96|$ cutoff value for statistical significance, thus normality of distribution was not assumed for participants' reported affect toward their instructor. Further, a Levene's test yielded a significant value $(\mathrm{p}=.02)$, thus homogeneity of variance was not assumed. Similarly, a Kolmogorov-Smirnov test $(p<.001)$ and a Shapiro-Wilk test $(p<.001)$ were also significant for participants' reported affect toward enrolling in future courses with their instructor. Both the $z$ score for kurtosis $(z=4.72)$ and the $z$ score for skewness $(z=-3.55)$ also fell beyond the $|1.96|$ cutoff value for statistical significance, thus normality of distribution was not assumed. In contrast, a Levene's test yielded a nonsignificant value ( $p=$ .386), suggesting that the assumption for homogeneity of variance was met for participants' reported affect toward enrolling in future courses with the same instructor.

The first ANOVA revealed significant differences in participants' reported levels of affect toward their instructor, Welch's $F(1,103)=7.836, p=.006, \eta^{2}=.058$, suggesting that participants assigned to the CTML-based lesson reported greater affect toward their instructor $(M$ $=6.74, S D=.48)$ than participants assigned to the lesson which was not taught in line with CTML-based best practices $(M=6.42, S D=.80)$. The second ANOVA also revealed significant differences in participants' reported levels of affect toward enrolling in future courses with their instructor, Welch's $F(1,117)=5.036, p=.027, \eta^{2}=.037$, suggesting that participants assigned to the CTML-based lesson reported greater affect toward enrolling in future courses with the same instructor $(M=6.41, S D=.90)$ than participants assigned to the lesson which was not taught in line with CTML-based best practices $(M=5.99, S D=1.23)$. Given these results, hypothesis three was supported.

Similar to the other analyses in this dissertation, the researcher was also interested in exploring differences in participants' reported affect toward their instructor across experimental 
conditions controlling for participants' interest in lesson content, familiarity with lesson content, and GPA. Results of the first one-way ANCOVA failed to reveal significant differences in participants' reported affect toward their instructor between experimental conditions, $F(1,117)=$ $2.59, p=.110, \eta_{\mathrm{p}}{ }^{2}=.022$, controlling for participants' interest in lesson content, familiarity with lesson content, and GPA. The estimated marginal means suggested that participants who attended the CTML-based Zoom lesson $\left(M_{\mathrm{adj}}=6.71\right)$ did not report significantly different affect toward their instructor than participants who attended the Zoom lesson which was not designed based upon CTML principles $\left(M_{\mathrm{adj}}=6.54\right)$. The second one-way ANCOVA similarly failed to reveal significant differences in participants' reported affect toward enrolling in future courses with their instructor between experimental conditions, $F(1,117)=2.26, p=.136, \eta_{\mathrm{p}}{ }^{2}=.019$, controlling for participants' interest in lesson content, familiarity with lesson content, and GPA. The estimated marginal means suggested that participants who attended the CTML-based Zoom lesson $\left(M_{\mathrm{adj}}=6.33\right)$ did not report significantly different affect toward enrolling in a future course with their instructor than participants who attended the Zoom lesson which was not designed based upon CTML principles $\left(M_{\mathrm{adj}}=6.06\right)$. 


\section{Summary}

Without covariates, the results of this dissertation indicate that the incorporation of CTML principles during instruction directly affected participants' performance on the postlesson test, such that participants who attended the CTML-based lesson $(M=69.86, S D=19.25)$ significantly outperformed those who attended the lesson which was not designed based upon CTML principles $(M=61.32, S D=22.39)$. Further, participants who attended the CTML-based lesson exhibited significantly greater affect toward their instructor, as well as affect toward enrolling in future courses with their instructor, than participants who attended the lesson which was not designed based on CTML principles of instructional design. In contrast, participants did not report having experienced significantly different levels of working memory overload between experimental conditions, nor did working memory overload mediate the effect of CTML-based instruction on students' post-lesson test scores as hypothesized (regardless of participants' reported self-regulation). 


\section{CHAPTER IV}

\section{Discussion}

The aim of this dissertation was to draw upon research grounded in CTML (Mayer, 2001) to identify specific ways in which university educators can enhance their online pedagogy using the affordances of Zoom. In particular, this dissertation explored the applicability and impact of an instructor's implementation of CTML's signaling (Mayer, 2001), embodiment (Mayer, 2005d), and generative activity principles (Mayer, 2021) during synchronous online teaching using specific Zoom features. Contrary to expectations, CTML-based instruction did not indirectly influence students' performance on a post-lesson test through reduced working memory overload. Rather, an instructor's implementation of CTML principles directly increased students' performance on a post-lesson test from roughly $61 \%$ to $70 \%$ on average. An instructor's implementation of CTML principles also positively influenced students' affect toward their instructor, such that students who attended a CTML-based lesson exhibited significantly greater affect toward both their instructor and the possibility of enrolling in future courses with their instructor than students who attended a standard online lesson. Altogether, the findings of this dissertation suggest that Zoom is well-suited to creating online learning environments grounded in CTML principles of instructional design and conducive to positive learning outcomes for students. This chapter discusses these findings and provides an interpretation of their implications for theory and teaching, as well as identifies limitations of this dissertation and how they might be addressed by future research.

\section{CTML Principles, Working Memory Overload, and Learning}

Contrary to expectations, an instructor's implementation of CTML's signaling, embodiment, and generative activity principles failed to significantly reduce the amount of 
working memory overload that students experienced during their assigned online lesson.

However, as predicted, CTML-based instruction nevertheless caused significant increases in students' learning as evidenced by their performance on a post-lesson test. Considering this finding, it might initially seem feasible to interpret that the impact of CTML-based instruction on student learning may be more immediate than the theory predicts. Recall that CTML characterizes learning as an ongoing process in which students are "active sense makers" (Mayer, 2001, p. 13) who must continuously engage with their information processing in order to select and organize novel information effectively, subsequently integrating that information with extant knowledge structures already stored in the long term memory. That is, CTML proposes that selection, organization, and integration constitute the causal mechanisms whereby learning occurs, thus CTML-based principles of instructional design are intended to assist students in navigating those mechanisms by reducing working memory overload. Students who attended a CTML-based online lesson did not perceive a reduction in working memory overload compared to students who attended a standard online lesson; yet nonetheless learned more, which is perplexing and seems to contradict the central propositions of CTML. However, such conclusions, although reasonable at first glance, may not be necessarily accurate.

Recall that, from the theoretical perspective of CTML, working memory is effectively synonymous with active consciousness (Mayer, 2021); it is limited both temporally and in terms of capacity, lasting only seconds and capable of processing an extremely finite number of pictorial or verbal representations at a single point in time (Baddeley, 1997; Mayer, 2001). Despite these limitations, Mayer (2021) contended that the working memory is where the lion's share of the sense-making processes which ultimately drives learning occurs. More specifically, it is within the working memory that learners' selection of sensory information, organization of 
sensory information into coherent verbal and pictorial models, and integration of models with pre-existing knowledge structures take place. In other words, CTML is based on the assumption that the step-by-step processes whereby learning occurs take place almost entirely within the finite scope of the working memory (i.e., many cognitively intensive processes are taking place in a limited space and a brief span of time). Given this, it is feasible that this process may seem almost instantaneous considering that selection, organization, and integration - as well as working memory overload - all coalesce to influence students' learning within the span of just a few seconds. That is, the fundamental processes through which CTML argues that learning occurs are likely still applicable when interpreting the direct effect of CTML-based instruction on students' learning observed in this dissertation, despite the lack of an observed indirect effect through working memory overload.

Indeed, the limited capacity, fleeting nature, and complexity of the working memory also make it plausible that students who participated in this dissertation may have been unable to accurately self-report the extent to which they experienced working memory overload during their assigned online lesson. The instrument used to measure working memory overload in this dissertation (Bolkan, 2017a) prompted students to respond to a series of statements related to their information processing following each lesson, asking students to indicate the extent to which they agreed or disagreed with each statement (e.g., "I felt flustered trying to keep up with the amount of information presented in this lesson;" "This lesson made me feel anxious because of the amount of information I was asked to learn all at one time") after their assigned online lesson had concluded. Asking students to report on their working memory overload after-the-fact and in aggregate may have ultimately been inappropriate for collecting data which would allow the researcher to characterize the moment-to-moment cognitive processes which occur within the 
working memory and mediate learning. Further, asking students to subjectively report on their own information processing, generally, may not be appropriate for examining the ways in which students learn. Wanzer and colleagues (2010), for example, suggested that subjectively measuring the cognitive processes associated with learning may be infeasible given their inherent complexity. Similarly, a meta-analysis by Sitzmann and colleagues (2010) concluded that selfassessments of learning are likely better suited for measuring students' affect regarding their learning experiences rather than the extent to which they truly learn. Echoing these sentiments, Zheng and Greenberg (2018) expressed concern related to the influence of response biases when employing self-report measurements of cognitive load - chiefly, that students' perceptions regarding their information processing and learning may not necessarily reflect their actual information processing or the extent to which they actually learn. While the test scores used to operationalize learning in this dissertation served as a more objective indicator of students' information processing, the subjectivity of the instrument used to measure working memory overload may have inhibited the researcher from capturing the extent to which working memory overload truly occurred from moment-to-moment.

Despite the lack of the hypothesized indirect effect for CTML-based instruction on learning through working memory overload, the findings of this dissertation nevertheless align with those of previous CTML research. Like previous studies exploring learning outcomes associated with an instructor's implementation of the signaling (Harp, \& Mayer, 1998; Li et al., 2019; Loman \& Mayer, 1983; Mautone \& Mayer, 2001; Mayer et al., 1984), embodiment (e.g., Li et al., 2019; Mayer \& DaPra, 2012; Wang et al., 2018), and generative activity principles (e.g., Fiorella \& Mayer, 2013, 2014; Parong \& Mayer, 2018; Peper \& Mayer, 1978, 1986; Ponce et al., 2018; Shrager \& Mayer, 1989), students learned more when an instructor strategically used 
Zoom features to incorporate cues highlighting the organization of essential lesson content, make themselves visible to students throughout the online lesson, and stimulate students' generative processing of important material. Reviewing relevant research exploring these three principles the majority of which have analyzed the main effects of CTML-based instruction on learning using either ANOVAs or $t$-tests - Mayer (2021) reported median effect sizes of $d=.51$ for the signaling principle, $d=.58$ for the embodiment principle, and $d=.71$ for the generative activity principle on student learning during multimedia instruction. In this dissertation, when all three principles were enacted together, the effect size was $d=.41$, consistent with the range of effect sizes reported by Mayer (2021) in his review. Although these effects were not observed when controlling for familiarity with lesson content, interest in lesson content, or GPA in subsequent analyses, those results should be interpreted with scrutiny given the relatively low number of students who participated in this dissertation, leading to a lack of statistical power in analyses including covariates (discussed further under Limitations and Future Directions).

How, specifically, might an instructor's use of Zoom features have contributed to improving learning outcomes for the students who attended the CTML-based online lesson? Firstly, an instructor's use of Zoom's annotation and screen-sharing features to highlight the organization of lesson material is a direct implementation of the signaling principle, which asserts that students will engage in more effective cognitive processing in multimedia lessons "when cues are added that highlight the organization of essential material" (Mayer, 2005c, p. 184). An instructor emphasizing specific information presented during a lesson by employing annotations such as circles, lines, arrows, squares, numbers, and text constitutes an act of signaling in that each annotation serves to direct students' attention to specific verbal or pictorial information, rather than placing the burden of deciding which information to pay attention to, or 
determining how information is interrelated, upon students (Mayer, 2021). The Zoom annotations employed during the CTML-based online lesson thus likely enhanced the instructor's organizational clarity (i.e., "the methods with which teachers use verbal, nonverbal, and visual resources to organize information for students," Titsworth \& Mazer, 2016, p. 119) by assisting students in selecting relevant information to attend to and organizing that information into coherent mental representations.

Second, the instructor remaining visible to students on camera for the duration of the CTML-based online lesson, and engaging in humanlike body movements while on camera, likely enhanced student learning by stimulating positive affective responses from students, as argued by Mayer (2014a). When students can see their instructors engaging in high-embodiment behaviors (i.e., behaviors similar those used during in-person interactions in the real world; Mayer, 2014a), it can "help establish a stronger social bond between the teacher and learner, causing the learner to try harder to make sense of the instructional message and thereby build a deeper learning outcome" (Mayer, 2021, p. 345). Indeed, the results of this dissertation revealed that students who attended the CTML-based online lesson exhibited significantly greater affect toward both their instructor and the possibility of enrolling in future courses with their instructor than students who attended a standard online lesson. As explained by Bolkan (2015) while advocating for the importance of students' affective experiences during learning, numerous studies (e.g., Allen et al., 2006; Bolkan \& Goodboy, 2015; Goodboy \& Bolkan, 2009; Goodboy et al., 2018; Knoster \& Goodboy, 2021; Rodriguez et al., 1996) have demonstrated that students' affective responses to instruction can facilitate a variety of important learning outcomes and impact students' motivation to put forth the mental effort necessary to engage in the active and ongoing cognitive processes which CTML asserts will increase meaningful learning. Given the 
discrepancies observed in students' reported affect toward their instructor and their overall learning between the CTML-based online lesson and standard online lesson, the findings of this dissertation lend further support to Mayer's (2014a) arguments concerning the salience of instructor embodiment in multimedia learning environments.

Third, the instructor's use of Zoom's polling feature during the CTML-based online lesson served to further guide students' selection, organization, and integration of important instructional material in a manner which was unavailable to the instructor of the standard online lesson. More specifically, each of the three polls provided a mechanism through which the instructor of the CTML-based online lesson could directly stimulate students' generative processing by enacting activities which prompted students to actively engage with material. Each poll posed a question (e.g., "Which of the three types of competence we just discussed is most closely related to how we work together with others in groups?"; "How we develop in the Establishing Identity vector is based on our development in which four other vectors? [Select all that apply]"; "Which of the seven key influences involves students and instructors interacting both inside and outside of the classroom?") which required students to reflect upon information presented earlier in the lesson, consider the ways in which that information related to other lesson material, and apply that information in responding to each individual poll. That is, each poll provided students with opportunities to self-test their understanding of information presented during the online lesson, as well as highlighted specific pieces of information which were particularly important for students to understand. Like previous research exploring the influence of self-testing on student learning (e.g., Johnson \& Mayer, 2009; Mayer, 1975, 1980; Sagerman \& Mayer, 1987), the results of this dissertation similarly position it as a viable generative activity which can prime more effective selection and organization of instructional 
content, as well as demonstrate that Zoom's polling feature is an effective tool for implementing this generative activity during synchronous online instruction.

\section{CTML Principles and Student Affect Toward Instructor}

As predicted, students who participated in a CTML-based online lesson reported significantly greater affect toward their instructor than students who participated in a standard online lesson. Moreover, students who attended a CTML-based online lesson exhibited significantly greater affect toward the possibility of enrolling in future courses with their instructor. These results are consistent with findings from previous CTML research, particularly those suggesting that instructors can stimulate a sense of social presence among students in multimedia classrooms by making themselves visible to students and moving in humanlike ways while teaching (e.g., Baylor \& Kim, 2009; Dunsworth \& Atkinson, 2010; Fiorella et al., 2019, 2020; Li et al., 2019; Lusk \& Atkinson, 2007; Mayer \& DaPra, 2012; Moreno et al., 2010; Stull

et al., 2018; Wang et al., 2018), as well as with instructional communication research suggesting that instructors who engage in nonverbally immediate behaviors (Andersen, 1979) can facilitate positive affective experiences for their students (e.g., Frymier \& Houser, 2000; Frymier et al., 2019; Mottet et al., 2006; Witt et al., 2004). The findings of this dissertation thus complement previous research in suggesting that an instructor's nonverbal behaviors may have significant implications for students' affective responses during instruction, even in remote learning environments.

In the CTML-based online lesson, students observed their instructor using hand gestures, looking into the camera, and employing a variety of facial expressions while speaking, providing the types of positive social cues which Mayer (2021) suggested are conducive to enhancing teacher-student relationships and priming student motivation. However, students' increased 
affect toward their instructor in the CTML-based online lesson may not be exclusively attributable to their instructor being visible on-screen. Recall that instructional communication research has consistently documented a positive relationship between clear teaching and student affect, such that students tend to exhibit greater affect toward instructors who communicate lesson content in comprehendible ways (e.g., Titsworth et al., 2015). Indeed, clear teaching has been demonstrated to impact a variety of affective outcomes for students, influencing students' sense of self-efficacy (LaBelle et al., 2013), positive (Titsworth et al., 2013) and negative (Mazer et al., 2014) emotions, receiver apprehension (Bolkan, 2016), and motivation (Avtgis, 2001; Bolkan et al., 2016; Chesebro \& McCroskey, 2001). Given this, it is feasible that students' heightened affect toward their instructor in the CTML-based online lesson may have also been stimulated by the instructor's use of Zoom's annotation, screen-sharing, and polling features to highlight important information for students to attend to and assist students in understanding that information during the lesson (i.e., the instructor's use of Zoom features to enhance instructional clarity).

Although CTML (Mayer, 2001) is primarily concerned with providing instructors guidance in creating multimedia lessons based upon the cognitive processes through which humans learn, extant CTML research nevertheless highlights the salient role that students' affective experiences can play in their information processing. Moreno (2005) asserted that affective elements of students' instructional experiences can have a profound effect on the extent to which students engage in the generative information processing which is fundamentally necessary for students to achieve meaningful learning outcomes. This sentiment was echoed by Mayer (2014b), who wrote that student affect is integral for learning insofar as it "initiates, maintains, and energizes the learner's effort to engage in learning processes" (p. 171). From a 
CTML-based perspective, learning is an active process (Mayer, 2001) - one in which students must put forth cognitive effort as they engage in making sense of novel information. Elements of instruction (e.g., embodiment, signaling, generative activity) which stimulate positive affective experiences for students thus facilitate generative processing by motivating students to pay attention to important instructional content (i.e., selecting) and exert the cognitive resources necessary to comprehend it (i.e., organizing and integrating; Mayer \& Estrella, 2014). Discussing the role of student affect in learning from a cognitive neuroscientific perspective, Mottet (2015) argued that "most of the thought processes that educators care about, including memory, learning, and creativity among others, critically involve both cognitions and emotions" (p. 508). Although the cognitive processes associated with learners selecting, organizing, and integrating novel information constitute the foundation of CTML, learners' affective experiences are nonetheless recognized as an important predictor of students achieving meaningful learning outcomes. That students who attended the CTML-based online lesson exhibited greater affect toward their instructor and outperformed students who attended a standard online lesson on a post-lesson test lends further support to arguments concerning the interdependence between students' emotional and cognitive outcomes. While differences in students' reported affect toward their instructor were not observed when controlling for familiarity, interest, or GPA, those results should be interpreted with skepticism in light of the relatively small number of students who participated in this dissertation, similar to this dissertation's analyses involving student learning (discussed further under Limitations and Future Directions).

\section{Implications for Theory and Online Teaching}

A major theoretical implication of this dissertation is that it affirms the positive impact of an instructor's implementation of CTML's signaling, embodiment, and generative activity 
principles on students' cognitive and affective learning outcomes in multimedia learning environments. Moreover, the findings of this dissertation reveal that these principles are conducive to enhancing student learning during synchronous remote instruction. While previous CTML research has primarily been based upon experimental manipulations of instructional design principles during text-based instruction (e.g., Harp \& Mayer, 1998; Loman \& Mayer, 1983; Mautone \& Mayer, 2001; Mayer et al., 1984; Ponce \& Mayer, 2014a, 2014b; Ponce et al., 2018; Wang et al., 2018; Xie et al., 2019) or during asynchronous lessons employing prerecorded materials (e.g., Fiorella \& Mayer, 2016a; Fiorella et al., 2019, 2020; Johnson \& Mayer, 2009; Li et al., 2019; Mayer \& DaPra, 2012; Wang et al., 2018), this dissertation illustrates the efficacy of CTML principles for enhancing students' learning during live online lessons. Given concerns voiced by previous researchers (e.g., Guo et al., 2014; Kuznekoff, 2020) as far as the degree to which asynchronous instruction may not necessarily be effective for consistently engaging remote online learners, this extension of CTML principles to synchronous learning contexts is important in that it illustrates ways in which remote learning platforms such as Zoom can be leveraged to capitalize upon strengths of live online classrooms which distinguish them from their asynchronous counterparts (McBrien et al., 2009; Offir et al., 2008; Peterson et al., 2018).

Another theoretical implication of this dissertation is that it lends further support to concerns voiced by previous researchers (e.g., Sitzmann et al., 2010; Wanzer et al., 2010; Zheng \& Greenberg, 2018) regarding the ability of self-report instruments to accurately measure the types of complex cognitive processes involved in students' learning. While this dissertation measured learning itself via an objective measure of students' ability to recall lesson content (and found that implementation of CTML principles enhanced students' learning), working memory 
overload (i.e., cognitive load) was assessed by prompting students who attended each online lesson to indicate, generally, the extent to which they felt cognitively overwhelmed while attempting to make sense of the information presented to them (and failed to detect significant changes in working memory overload following an instructor's implementation of CTML principles). Mazer and Graham (2019) noted that self-perceptions of one's own learning may be influenced by a variety of personal biases - creating discrepancies between the extent to which students believe that they learn and the degree to which they actually do. Further, the cognitive processes which fundamentally drive learning might be so innately fluid and complex that students may lack sufficient self-awareness to accurately recognize or describe those processes to begin with, particularly when asked to do so in hindsight or in the aggregate. That this dissertation failed to observe significant differences in students' working memory overload between experimental conditions, yet students who attended the CTML-based lesson condition learned more, suggests that future researchers interested in exploring the influence of working memory overload on students' overall learning should be careful in considering the ways in which they measure the underlying cognitive processes assumed to ultimately drive learning.

Given that literature suggests many instructors unexpectedly teaching online following the COVID-19 pandemic struggled in adapting to online teaching (e.g., Hess, 2020; Huber \& Helm, 2020; Kali et al., 2011; Means \& Neisler, 2020; Rapanta et al., 2020), it is also important to consider potential practical implications of this dissertation for teachers in online classrooms using Zoom. First, instructors teaching via Zoom (or similar remote conferencing software) should make strategic efforts to avail themselves of program features that can be used to enact signaling during online instruction. While the instructor of the CTML-based Zoom lesson in this dissertation incorporated signaling by creating shapes (e.g., circles, arrows), employing 
highlighting using the "draw" feature, and including additional numbers and text to provide organizational cues while sharing their screen, this does not represent the full array of ways in which instructors might use Zoom's annotation function to assist students in selecting and organizing information. Zoom's annotation feature also allows instructors to "stamp" specific pieces of information with check marks, stars, arrows, exes, hearts, and question marks, as well as "spotlight" specific information using colorful icons which can be substituted for the instructor's mouse cursor on-screen (Zoom, n.d.). Further, instructors can modify the formatting of annotations in a variety of ways, such as by adjusting the thickness of lines, bolding or italicizing numbers and text, increasing or decreasing font size, or employing different colors for specific annotations to assist students in differentiating between them (Zoom, n.d.). In doing so, however, instructors should be mindful that they do not employ so many annotations that they inadvertently violate CTML's coherence principle (Mayer, 2001) by drawing students' attention away from important lesson material, thereby facilitating extraneous processing. As evidenced by previous research (e.g., Sung \& Mayer, 2012), instructors' signaling attempts must be relevant to instructional goals in order to be effective - that is, instructors should be deliberate in ensuring that the annotations they use while teaching via Zoom direct students' attention toward, rather than away from, important core content.

Second, instructors should make themselves visible to their students when teaching online by turning and keeping their cameras on during instruction. Given that Zoom's default settings allow on-camera instructors to remain visible to students even while sharing their screens (presenting a live video of instructors in a smaller window located in the corner of their students' screens), allowing students to see their instructor should be as straightforward as activating one's camera at the beginning of an online lesson (Zoom, n.d.). While making oneself 
visible to students may be relatively straightforward, however, instructors should also be mindful of their nonverbal behaviors while on camera. As originally emphasized by Mayer (2014a), students simply being able to see their instructor may not necessarily be enough to stimulate positive affective responses in-and-of itself. Rather, those affective responses are largely influenced by the extent to which students perceive their instructors' nonverbal behaviors as high-embodiment (Mayer, 2014a) or immediate (Andersen, 1979). Instructors can facilitate these perceptions by making a conscious effort to look into their cameras while speaking to students as a substitute for in-person eye-contact, using hand gestures and physical movement to emphasize instructional content as if they were conveying information to students face-to-face, and employing appropriate facial expressions when communicating with students (e.g., smiling). While doing so, instructors should carefully consider the timing and purpose of their nonverbal behaviors. As observed by Stull and colleagues (2018), "the execution of these behaviors by the instructor could have either positive or negative consequences depending on how they are synchronized with the delivery of described, written, or drawn information" (p. 26). Engaging in inappropriately timed nonverbal behaviors which draw students' attention toward the instructor and away from important lesson content can thus potentially inhibit students' learning by imposing an additional distraction, creating split-attention effects. Similarly, instructors may benefit from being proactive in ensuring that they can teach their online lessons in quiet rooms with minimal background noises or distractions (e.g., pets, clutter, decorations, individuals other than the instructor), as these may also divert students' attention from important information presented in synchronous remote classrooms.

Third, instructors teaching via Zoom should leverage program features strategically to incorporate activities into their online lessons which stimulate students' generative processing of 
information. That is, instructors teaching online should use Zoom features to prompt their students to engage in active, as opposed to passive, learning. In the CTML-based lesson of this dissertation, the instructor utilized Zoom's polling feature to prompt students to engage in selftesting (i.e., studying information and completing practice assessments; Mayer, 2021) - assisting students in recognizing particularly important pieces of information presented during the lesson (i.e., selecting), understanding how that information was interrelated (i.e., organizing), and remembering that information later (i.e., integrating; Mayer, 2021). However, this is just one way in which instructors might use the various affordances of Zoom to implement generative activities in their online classrooms. For example, instructors teaching via Zoom could provide students with opportunities for self-explaining (i.e., creating one's own explanations of lesson content) or teaching (i.e., providing other learners with explanations of lesson content) by utilizing Zoom's breakout room feature to split students into smaller discussion groups (Zoom, n.d.), facilitating students' active engagement with both lesson content and other students attending an online lesson. Instructors might also consider ways in which they can allow their students to create their own annotations during lessons by adjusting Zoom's screen-sharing settings (Zoom, n.d.), stimulating students' generative processing by providing opportunities to engage in mapping (i.e., creating spatial representations of important information or terms) or drawing (i.e., creating illustrations which depict instructional content) during instruction, or prompt their students to guide their own use of Zoom annotations to create visual representations of instructional content via microphone or chat. Utilizing Zoom features in any of these ways may stimulate action from otherwise passive students during online instruction, thereby promoting greater engagement with material and facilitating deeper learning (Fiorella \& Mayer, 2015, 2016b). 
While instructors might feasibly enhance their students' learning by employing any of these strategies during Zoom-based instruction, the extent to which they are able to do so effectively is undoubtedly contingent upon their familiarity and comfort teaching via Zoom (or teaching online, in general). Put simply, instructors cannot reasonably be expected to capitalize on the affordances of Zoom if they are unaware of what those affordances are or how to best incorporate them during online lessons. Given that literature suggests instructors at colleges and universities have struggled in adapting to online teaching chiefly due to lack of pedagogical knowledge and experience in remote classrooms (Huber \& Helm, 2020; Kali et al., 2011; Rapanta et al., 2020), program administrators should be proactive in considering ways in which they can assist teaching staff in overcoming these obstacles. "Universities, now more than ever, should invest in teacher professional development of their faculty, for them to be updated on effective pedagogical methods with or without the use of online technologies" (Rapanta et al., 2020, p. 942).

\section{Limitations and Future Directions}

The first limitation of this dissertation is a lack of statistical power in analyses given the relatively small number of participants in this study. As described previously, data obtained from 20 of the total 160 students who participated in this dissertation were omitted due to participants having either (1) been enrolled in previous courses with the instructor of the experimental online lessons or (2) participated in multiple data collections - resulting in a final sample size of 140 . This is problematic for multiple reasons. First, it inhibited the researcher from conducting confirmatory factor analyses (CFAs) on the scales used in this dissertation prior to hypothesis testing. Muthén and Muthén (2002) reported, "For the simplest CFA model with normally distributed continuous factor indicators and no missing data, a sample size of 150 is needed for 
power of .81” (p. 9), while Kline (2005) recommended a minimum of 100 cases or observations per group in CFAs employing multi-group modeling. Second, the small sample size of this dissertation calls into question the results of the mediation analyses used to assess the extent to which CTML-based principles of instructional design influenced learning through students' working memory overload. Fritz and MacKinnon (2007) reported that a minimum sample size of 162 is required to obtain adequate statistical power $(.80, \alpha=.05)$ to detect a small to moderate mediated effect $(a$ path $=.26, b$ path $=.26)$ in mediation models such as those tested in this dissertation, suggesting that this dissertation's sample size of 140 may have been insufficient.

Third, the lack of participants in this dissertation calls into question the accuracy of analyses including covariates (interest, familiarity, GPA) employed subsequently to hypothesis testing. Brysbaert (2019) suggested that researchers comparing group means in experiments should aim to recruit a minimum sample size of 200 participants (split into two groups, each comprised of 100 participants). When including additional independent variables as covariates, such as when conducting an ANCOVA, VanVoorhis and Morgan (2007) reviewed best practices in calculating sample size and recommended that analyses include at least 30 additional participants per covariate. Considering both pieces of guidance together, controlling for interest (cognitive and emotional), familiarity, and GPA while exploring differences emerging from CTML-based vs. standard online teaching warrants a sample size of roughly 320 participants to obtain prerequisite statistical power - double the size of the sample which participated in this dissertation. Although differences in students' learning and affect based on CTML-based instruction were detected despite this dissertation's analyses being statistically underpowered, the small size of the sample in this dissertation nevertheless inhibits a more thorough examination of those differences when controlling for other potential predictors. 
The fourth limitation of this dissertation involves the types of questions used to assess students' learning on the post-lesson test. While the multiple-choice questions which comprised the post-lesson test provided a means of assessing students' basic information recall, they did not measure the types of meaningful learning outcomes which constitute the penultimate goal of CTML-based instructional design (Mayer, 2001). Meaningful learning, according to Mayer (2001), takes place when learners exhibit both retention and understanding of instructional material. Mayer (2021) wrote that deeper understanding of content is best assessed using transfer questions prompting students to apply newly learned information while solving novel problems, such as by posing open-ended troubleshooting (i.e., questions which prompt students to identify and explain problems in a system or process), redesign (i.e., questions which prompt students to modify a system or process to meet a new requirement), prediction (i.e., questions which prompt students to anticipate potential outcomes associated with changing a component of a system or process), or conceptual questions (i.e., questions which prompt students to explain how or why a system or process behaves the way that it does). While the multiple-choice questions included in this dissertation's post-lesson test are appropriate for assessing students' recall and recognition of lesson content, "tests of retention such as recall or recognition tests provide a limited view of what someone knows" (Mayer, 2021, p. 98). Given this, this dissertation cannot speak to the extent that an instructor's implementation of CTML-based principles of instructional design may have affected students' deeper understanding of instructional content beyond basic memorization. Future researchers interested in exploring how learning occurs during Zoombased instruction should thus carefully consider ways in which they can employ both retention and transfer questions to measure students' deeper learning outcomes.

The fifth limitation of this dissertation is that it only explored the applicability and impact 
of CTML-based instructional design within the context of a single online lesson. As such, the findings of this dissertation may not necessarily be representative of instructional outcomes that instructors might facilitate in remote classrooms in the real world, which students usually connect to repeatedly (e.g., throughout a semester or school year) as opposed to only once. Indeed, it stands to reason that an instructor's implementation of CTML-based instructional design across the span of an entire semester may influence learning differently than it does within the finite scope of an isolated online lesson. Similar to the ways in which other instructional strategies and behaviors (e.g., immediacy; Allen et al., 2006) have been suggested to exert a cumulative effect on students' learning experiences over time, an instructors' implementation of CTML-based principles in online learning environments could potentially influence long-term affective and cognitive outcomes. Given this, future research could greatly expand upon the findings of this dissertation by examining the effects of CTML-based instructional design on students' learning experiences over time.

The sixth limitation of this dissertation involves how the researcher measured students' experiences with cognitive load during online instruction. As discussed previously, students were presented with a series of four self-report questions adapted from the working memory overload subscale of Bolkan's (2017a) Clarity Indicators Scale after attending their assigned online lesson, which may have been inappropriate for examining the complex, finite cognitive processes which drive students' learning (e.g., Mazer \& Graham, 2019; Sitzmann et al., 2010; Wanzer et al., 2010; Zheng \& Greenberg, 2018). Future researchers interested in exploring students' working memory overload (or lack thereof) during synchronous online learning may thus benefit from considering alternative methods for measure cognitive load, such as employing efficiency measures (which employ both subjective and task performance indicators of cognitive load), 
imposing secondary tasks (to assess the amount of working memory processing still available subsequent to students' completing a primary task), or using physiological measures (e.g., functional magnetic resonance imaging, electroencephalography; Sweller et al., 2011). Future researchers interested in delineating specific types of cognitive load (e.g., extraneous, intrinsic, germane) may also benefit from employing instruments such as the NASA Task Load Index (Hart \& Staveland, 1988); a multidimensional scale assessing the (1) mental, (2) physical, (3) temporal, (4) performance, (5) effort, and (6) frustration-related demands associated with completing a specific task. In doing so, researchers may be able to make more nuanced comparisons between the different types of cognitive processes which facilitate students' learning in remote classrooms.

Future research may also benefit from exploring how other Zoom features might enhance or inhibit students' learning during synchronous online instruction. While this dissertation explored the applicability and impact of an instructor enacting CTML-based principles via Zoom's annotation, screen-sharing, and polling features, other Zoom features such as breakout rooms or chat might further influence students' online learning experiences in different ways. Similarly, future researchers interested in examining students' learning during synchronous online instruction might explore different strategies or techniques as far as how to best utilize specific Zoom features. In this dissertation, for example, an instructor either appeared on camera for the entirety of an online lesson (in the treatment condition) or was not visible to students at any point during an online lesson (in the control condition). Future researchers could compare the influence of an instructor appearing on camera at different times or in different intervals during a synchronous online lesson, assessing the degree to which an instructor being visible at particular points in time or while presenting specific pieces of information may induce the types 
of split-attention effects described by Stull and colleagues (2018). As written by Mayer (2021), "future research is needed to better pinpoint the boundary conditions for each [CTML] principle" (p. 410) despite the abundance of studies conducted to date, thus further study concerning best practices for enacting each principle is warranted. 


\section{Summary}

This dissertation explored the applicability and impact of CTML-based principles of instructional design to Zoom-based online teaching environments. Omitting covariates, an instructor's strategic use of Zoom features to enact CTML's signaling, embodiment, and generative activity principles directly enhanced students' performance on a post-lesson test, as well as stimulated greater affect among students toward the instructor of their online lesson. Altogether, these findings characterize Zoom as well-suited to creating online learning environments grounded in CTML principles of instructional design which are conducive to positive learning outcomes for students. In particular, the results of this dissertation suggest that instructors' utilization of Zoom's annotation, screen-share, and polling features can offer significant improvements for their students' online synchronous learning experiences. 


\section{References}

Adesope, O. O., \& Nesbit, J. C. (2012). Verbal redundancy in multimedia learning environments: A meta-analysis. Journal of Educational Psychology, 104(1), 250-263. https://doi.org/10.1037/a0026147

Allen, M. (2017). The Sage encyclopedia of communication research methods. Sage. https://dx.doi.org/10.4135/9781483381411

Allen, M., Witt., P. L., \& Wheeless, L. R. (2006). The role of teacher immediacy as a motivational factor in student learning: Using meta-analysis to test a causal model. Communication Education, 55(1), 21-31. https://doi.org/10.1080/03634520500343368

Anderson, G. (2020, April 13). Feeling shortchanged. Inside Higher Ed. https://www.insidehighered.com/news/2020/04/13/students-say-online-classes-arentwhat-they-paid

Anderson, L. W., Krathwohl, D. R., Airasian, P. W., Cruikshank, K. A., Mayer, R. E., Pintrich, P. R., Raths, J., \& Wittrock, M. C. (2001). A taxonomy of learning for learning, teaching, and assessing: A revision of Bloom's taxonomy of educational objectives. Longman.

Atkinson, R. K. (2002). Optimizing learning from examples using animated pedagogical agents. Journal of Educational Psychology, 94(2), 416-427. https://doi.org/10.1037/0022-0663.94.2.416

Atkinson, R. K., Mayer, R. E., \& Merrill, M. M. (2005). Fostering social agency in multimedia learning: Examining the impact of an animated agent's voice. Contemporary Educational Psychology, 30(1), 117-139. https://doi.org/10.1016/j.cedpsych.2004.07.001

Austin, K. A. (2009). Multimedia learning: Cognitive individual differences and display design techniques predict transfer learning with multimedia learning modules. Computers \& 
Education, 53(4), 1339-1354. http://doi.org/10.1016/j.compedu.2009.06.017

Avtgis, T. A. (2001). Affective learning, teacher clarity, and student motivation as a function of attributional confidence. Communication Research Reports, 18(4), 345-353. https://doi.org/10.1080/08824090109384815

Ayres, P. (2006). Impact of reducing intrinsic cognitive load on learning in a mathematical domain. Applied Cognitive Psychology, 20(3), 287-298. http://doi.org/10.1002/acp.1245

Ayres, P., \& Sweller, J. (2014). The split-attention principle in multimedia learning. In R. E. Mayer (Ed.), The Cambridge handbook of multimedia learning (2 ${ }^{\text {nd }}$ ed., pp. 206-226). Cambridge University Press. https://doi.org/10.1017/cbo9781139547369.011

Baddeley, A. D. (1992). Working memory. Science, 255(5044), 556-559. http://doi.org/10.1126/science.1736359

Baddeley, A. D. (1997). Human memory: Theory and practice. Psychology Press. https://doi.org/10.1016/s0145-2134(00)00166-6

Baddeley, A. D. (1999). Essentials of human memory. Allyn \& Bacon. https://doi.org/10.4324/9780203345160

Baggett, P. (1984). Role of temporal overlap of visual and auditory material in forming dual media associations. Journal of Educational Psychology, 76(3), 408-417. http://doi.org/10.1037/0022-0663.76.3.408

Baggett, P., \& Ehrenfeucht, A. (1983). Encoding and retaining information in the visuals and verbals of an educational movie. Educational Technology Research and Development, 31(1), 23-32. http://doi.org/10.1007/BF02765208

Baylor, A. L., \& Kim, S. (2009). Designing nonverbal communication for pedagogical agents: When less is more. Computers in Human Behavior, 25(2), 450-457. 
http://doi.org/10.1016/j.chb.2008.10.008

Begg, I. (1972). Recall of meaningful phrases. Journal of Verbal Learning and Verbal Behavior, 11(4), 431-439. http://doi.org/10.1016/S0022-5371(72)80024-0

Biard, N., Cojean, S., \& Jamet, E. (2018). Effects of segmentation and pacing on procedural learning by video. Computers in Human Behavior, 89(1), 411-417. http://doi.org/10.1016/j.chb.2017.12.002

Blagg, K. (2020). How might COVID-19 affect Fall 2020 higher education enrollment? Modeling the effects of different enrollment scenarios. Urban Institute.

Bodemer, D., Ploetzner, R., Feuerlein, I., \& Spada, H. (2004). The active integration of information during learning with dynamic and interactive visualisations. Learning and Instruction, 14(3), 325-341. http://doi.org/10.1016/j.learninstruc.2004.06.006

Bolkan, S. (2015). Students' affective learning as affective experience: Significance, reconceptualization, and future directions. Communication Education, 64(4), 502-505. https://doi.org/10.1080/03634523.2015.1058963

Bolkan, S. (2016). The importance of instructor clarity and its effect on student learning: Facilitating elaboration by reducing cognitive load. Communication Reports, 29(3), 152162. http://doi.org/10.1080/08934215.2015.1067708

Bolkan, S. (2017a). Development and validation of the clarity indicators scale. Communication Education, 66(1), 19-36. http://doi.org/10.1080/03634523.2016.1202994

Bolkan, S. (2017b). Instructor clarity, generative processes, and mastery goals: Examining the effects of signaling on student learning. Communication Education, 66(4), 385-401. https://doi.org/10.1080/03634523.2017.1313438

Bolkan, S. (2019). Facilitating student attention with multimedia presentations: examining the 
effects of segmented PowerPoint presentations on student learning. Communication Education, 68(1), 61-79. https://doi.org/10.1080/03634523.2018.1517895

Bolkan, S., \& Goodboy, A. K. (2015). Exploratory theoretical tests of the instructor humorstudent learning link. Communication Education, 64(1), 45-64. https://doi.org/10.1080/03634523.2014.978793

Bolkan, S., \& Goodboy, A. K. (2019). Examples and the facilitation of student learning: Should instructors provide examples or should students generate their own? Communication Education, 68(3), 287-307. http://doi.org/10.1080/03634523.2019.1602275

Bolkan, S., Goodboy, A. K., \& Kelsey, D. M. (2016). Instructor clarity and student motivation: Academic performance as a product of students' ability and motivation to process instructional material. Communication Education, 65(2), 129-148. http://doi.org/10.1080/03634523.2015.1079329

Bolkan, S., Goodboy, A. K., \& Myers, S. A. (2017). Conditional processes of effective instructor communication and increases in students' cognitive learning. Communication Education, 66(2), 129-147. http://doi.org/10.1080/03634523.2016.1241889

Boucheix, J. M., \& Guignard, H. (2005). What animated illustrations conditions can improve technical document comprehension in young students? Format, signaling and control of the presentation. European Journal of Psychology of Education, 20(4), 369-388. http://doi.org/10.1007/BF03173563

Boucheix, J. M., \& Schneider, E. (2009). Static and animated presentations in learning dynamic mechanical systems. Learning and Instruction, 19(2), 112-127. http://doi.org/10.1016/j.learninstruc.2008.03.004

Brysbaert, M. (2019). How many participants do we have to include in properly powered 
experiments? A tutorial of power analysis with reference tables. Journal of Cognition, 2(1), 16. http://doi.org/10.5334/joc.72

Bush, A. J., Kennedy, J. J., \& Cruickshank, D. R. (1977). An empirical investigation of teacher clarity. Journal of Teacher Education, 28(2), 53-58. http://doi.org/10.1177/002248717702800216

Carillo, C., \& Flores, M. A. (2020). COVID-19 and teacher education: A literature review of online teaching and learning practices. European Journal of Teacher Education, 43(4), 466-487. http://doi.org/10.1080/02619768.2020.1821184

Catrambone, R. (1994). Improving examples to improve transfer to novel problems. Memory \& Cognition, 22(5), 606-615. http://doi.org/10.3758/BF03198399

Catrambone, R. (1995). Aiding subgoal learning: Effects on transfer. Journal of Educational Psychology, 87(1), 5-17. http://doi.org/10.1037/0022-0663.87.1.5

Catrambone, R. (1998). The subgoal learning model: Creating better examples so that students can solve novel problems. Journal of Experimental Psychology: General, 127(4), 355376. http://doi.org/10.1037/0096-3445.127.4.355

Chandler, P., \& Sweller, J. (1991). Cognitive load theory and the format of instruction. Cognition and Instruction, 8(4), 293-332. http://doi.org/10.1207/s1532690xci0804_2

Chandler, P., \& Sweller, J. (1996). Cognitive load while learning to use a computer program. Applied Cognitive Psychology, 10(2), 151-170. http://doi.org/10.1002/(SICI)1099-0720(199604)10:2<151::AID-ACP380>3.0.CO;2-U

Chesebro, J. L. (2003). Effects of teacher clarity and nonverbal immediacy on student learning, receiver apprehension, and affect. Communication Education, 52(2), 135-147. 
http://doi.org/10.1080/03634520302471

Cerpa, N., Chandler, P., \& Sweller, J. (1996). Some conditions under which integrated computerbased training software can facilitate learning. Journal of Educational Computing Research, 15(4), 345-367. http://doi.org/10.2190/MG7X-4J8N-CKYR-P06T

Chen, C., \& Yen, P. (2019). Learner control, segmenting, and modality effects in animated demonstrations used as the before-class instructions in the flipped classroom. Interactive Learning Environments. http://doi.org/10.1080/10494820.2019.1572627

Chesebro, J. L., \& McCroskey, J. C. (1998). The development of the teacher clarity short inventory (TCSI) to measure clear teaching in the classroom. Communication Research Reports, 15(3), 262-266. http://doi.org/10.1080/08824099809362122

Chesebro, J. L., \& McCroskey, J. C. (2001). The relationship of teacher clarity and immediacy with student state receiver apprehension, affect, and cognitive learning. Communication Education, 50(1), 59-68. https://doi.org/10.1080/03634520109379232

Chickering, A. W. (1969). Education and identity. Jossey-Bass.

Chun, D. M., \& Plass, J. L. (1996). Effects of multimedia annotations on vocabulary acquisition. Modern Language Journal, 80(2), 183-198. http://doi.org/10.2307/328635

Chung, K. K. H. (2007). Presentation factors in the learning of Chinese characters: The order and position of Hanyu pinyin and English translations. Educational Psychology, 27(1), 1-20. http://doi.org/10.1080/01443410601061306

Cierniak, G., Scheiter, K., \& Gerjets, P. (2009). Explaining the split-attention effect: Is the reduction of extraneous cognitive load accompanied by an increase in germane cognitive load? Computers in Human Behavior, 25(2), 315-324.

http://doi.org/10.1016/j.chb.2008.12.020 
Clarke, T., Ayres, P., \& Sweller, J. (2005). The impact of sequencing and prior knowledge on learning mathematics through spreadsheet applications. Educational Technology Research and Development, 53(3), 15-24. http://doi.org/10.1007/BF02504794

Colliot, T. \& Jamet, É. (2018). Understanding the effects of a teacher video on learning from a multimedia document: An eye-tracking study. Educational Technology, Research, and Development, 66(6), 1415-1433. http://doi.org/10.1007/s11423-018-9594-x

Cook, L. K., \& Mayer, R. E. (1988). Teaching readers about the structure of scientific text. Journal of Educational Psychology, 80(4), 448-456. http://doi.org/10.1037/0022-0663.80.4.448

Craig, S. D., Gholson, B., \& Driscoll, D. M. (2002). Animated pedagogical agents in multimedia educational environments: Effects of agent properties, picture features and redundancy. Journal of Educational Psychology, 94(2), 428-434. http://doi.org/10.1037/0022-0663.94.2.428

Cruickshank, D. R. (1985). Applying research on teacher clarity. Journal of Teacher Education, 36(2), 44-48. http://doi.org/10.1177/002248718503600210

Dabbagh, N., \& Kitsantas, A. (2012). Personal learning environments, social media, and selfregulated learning: A natural formula for connecting formal and informal learning. The Internet and Higher Education, 15(1), 3-8. https://doi.org/10.1016/j.iheduc.2011.06.002

Dilley, M. G., \& Paivio, A. (1968). Pictures and words as stimulus and response items in pairedassociate learning of young children. Journal of Experimental Child Psychology, 6(2), 231-240. http://doi.org/10.1016/0022-0965(68)90087-8

Dixson, M. D., Greenwell, M. R., Rogers-Stacy, C., Weister, T., \& Lauer, S. (2017). Nonverbal immediacy behaviors and online student engagement: Bringing past instructional 
research into the present virtual classroom. Communication Education, 66(1), 37-53. http://doi.org/10.1080/03634523.2016.1209222

Domagk, S. (2010). Do pedagogical agents facilitate learner motivation and learning outcomes? The role of the appeal of agent's appearance and voice. Journal of Media Psychology: Theories, Methods, and Applications, 22(2), 84-97. http://doi.org/10.1027/1864-1105/a000011

Dunsworth, Q., \& Atkinson, R. K. (2007). Fostering multimedia learning of science: Exploring the role of an animated agent's image. Computers \& Education, 49(3), 677-690. http://doi.org/10.1016/j.compedu.2005.11.010

Dutke, S., Grefe, A. C., \& Leopold, C. (2016). Learning from scientific texts: Personalizing the text increases transfer performance and task involvement. European Journal of Psychology of Education, 31(4), 499-513. http://doi.org/10.1007/s10212-015-0281-6

Dworak, A. (2020). United States university enrollment numbers during the COVID-19 pandemic recession. In R. D. Keysser (Ed.), Perspectives on the new normal: Post COVID-19. St. Mary’s University.

Eitel, A., Scheiter, K., \& Schüler, A. (2013). How inspecting a picture affects processing of text in multimedia learning. Applied Cognitive Psychology, 27(4), 451-461. http://doi.org/10.1002/acp.2922

Evans, N. J., Forney, D. S., Guido, F. M, Patton, L. D., \& Renn, K. A. (2010). Student development in college ( $2^{\text {nd }}$ ed.). Jossey-Bass.

Farris, K. L., Houser, M. L., \& Hosek, A. M. (2018). Historical roots and trajectories of instructional communication. In M. L. Houser \& A. M. Hosek (Eds.), Handbook of instructional communication: Rhetorical and relational perspectives ( $2^{\text {nd }}$ ed., pp. 1-20). 
Routledge. https://doi.org/10.4324/9781315189864

Fiorella, L., \& Mayer, R. E. (2012). Paper-based aids for learning with a computer-based game. Journal of Educational Psychology, 104(4), 1074-1082. http://doi.org/10.1037/a0028088

Fiorella, L., \& Mayer, R. E. (2013). The relative benefits of learning by teaching and teaching expectancy. Contemporary Educational Psychology, 38(4), 281288. http://doi.org/10.1016/j.cedpsych.2013.06.001

Fiorella, L., \& Mayer, R. E. (2014). Role of expectations and explanations in learning by teaching. Contemporary Educational Psychology, 39(2), 75-85. http://doi.org/10.1016/j.cedpsych.2014.01.001

Fiorella, L., \& Mayer, R. E. (2015). Learning as a generative activity: Eight learning strategies that promote understanding. Cambridge University Press. http://doi.org10.1017/CBO9781107707085

Fiorella, L., \& Mayer, R. E. (2016a). Effects of observing the instructor draw diagrams on learning from multimedia messages. Journal of Educational Psychology, 108(4), 528546. http://doi.org/10.1037/edu0000065

Fiorella, L., \& Mayer, R. E. (2016b). Eight ways to promote generative learning. Educational Psychology Review, 28(4), 717-741. http://doi.org/10.1007/s10648-015-9348-9

Fiorella, L., \& Mayer, R. E. (2017). Spontaneous spatial strategy use in learning from scientific text. Contemporary Educational Psychology, 49(1), 66-79. http://doi.org/10.1016/j.cedpsych.2017.01.002

Fiorella, L., Stull, A. T., Kuhlmann, S., \& Mayer, R. E. (2019). Instructor presence in video lectures: The role of dynamic drawings, eye contact, and instructor visibility. Journal of 
Educational Psychology, 111(7), 1162-1171. http://doi.org/10.1037/edu0000325

Fiorella, L., Stull, A. T., Kuhlmann, S., \& Mayer, R. E. (2020). Fostering generative learning from video lessons: Benefits of instructor-generated drawings and learner-generated explanations. Journal of Educational Psychology, 112(5), 895-906. http://doi.org/10.1037/edu0000408

Fiorella, L., van Gog, T., Hoogerheide, V., \& Mayer, R. E. (2017). It's all a matter of perspective: Viewing first-person video modeling examples promotes learning of an assembly task. Journal of Educational Psychology, 109(5), 653-665. http://doi.org/10.1037/edu0000161

Fletcher, J. D., \& Tobias, S. (2005). The multimedia principle. In R. E. Mayer (Ed.), The Cambridge handbook of multimedia learning (pp. 117-134). Cambridge University Press. https://doi.org/10.1017/cbo9780511816819.008

Florax, M., \& Ploetzner, R. (2010). What contributes to the split-attention effect? The role of text segmentation, picture labelling, and spatial proximity. Learning and Instruction, 20(3), 216-224. http://doi.org/10.1016/j.learninstruc.2009.02.021

Frechette, C., \& Moreno, R. (2010). The roles of animated pedagogical agents' presence and nonverbal communication in multimedia learning environments. Journal of Media Psychology: Theories, Methods, and Applications, 22(2), 61-72. http://doi.org/10.1027/1864-1105/a000009

Frisby, B. N., \& Martin, M. M. (2010). Instructor-student and student-student rapport in the classroom. Communication Education, 59(2), 146-164. http://doi.org/10.1080/03634520903564362

Fritz, M. S., \& MacKinnon, D. P. (2007). Required sample size to detect the mediated effect. 
Psychological Science, 18(3), 233-239.

https://doi.org/10.1111\%2Fj.1467-9280.2007.01882.x

Frymier, A. B. (1994). A model of immediacy in the classroom. Communication Quarterly, 42(2), 133-144. http://doi.org/10.1080/01463379409369922

Frymier, A. B., Goldman, Z. W., \& Claus, C. J. (2019). Why nonverbal immediacy matters: A motivation explanation. Communication Quarterly, 67(5), 526-539. http://doi.org/10.1080/01463373.2019.1668442

Frymier, A. B., \& Houser, M. L. (2000). The teacher-student relationship as an interpersonal relationship. Communication Education, 49(3), 207-219. http://doi.org/10.1080/03634520009379209

Garner, R., Alexander, P. A., Gillingham, M. G., Kulikowich, J. M., \& Brown, R. (1991). Interest and learning from text. American Educational Research Journal, 28(3), 643-659. http://doi.org/10.2307/1163152

Garner, R., Gillingham, M. G., \& White, C. S. (1989). Effects of "seductive details" on macroprocessing and microprocessing in adults and children. Cognition and Instruction, 6(1), 41-57. http://doi.org/10.1207/s1532690xci0601_2

Gegner, J. A., Mackay, D. H. J., \& Mayer, R. E. (2009). Computer-supported aids to making sense of scientific articles: Cognitive, motivational, and attitudinal effects. Educational Technology Research and Development, 57(1), 79-97. http://doi.org/10.1007/s11423-008-9088-3

Gerjets, P., Scheiter, K., \& Catrambone, R. (2004). Designing Instructional Examples to Reduce Intrinsic Cognitive Load: Molar versus Modular Presentation of Solution Procedures. Instructional Science, 32(1-2), 33-58. 
http://doi.org/10.1023/B:TRUC.0000021809.10236.71

Gerjets, P., Scheiter, K., \& Catrambone, R. (2006). Can learning from molar and modular worked examples be enhanced by providing instructional explanations and prompting self-explanations? Learning and Instruction, 16(2), 104-121. http://doi.org/10.1016/j.learninstruc.2006.02.007

Ginns, P. (2005). Meta-analysis of the modality effect. Learning and Instruction, 15(4), 313-331. http://doi.org/10.1016/j.learninstruc.2005.07.001

Ginns, P. (2006). Integrating information: A meta-analysis of the spatial contiguity and temporal contiguity effects. Learning and Instruction, 16(6), 511-525. http://doi.org/10.1016/j.learninstruc.2006.10.001

Ginns, P., \& Fraser, J. (2010). Personalization enhances learning anatomy terms. Medical Teacher, 32(9), 776-778. http://doi.org/10.3109/01421591003692714

Ginns, P., Martin, A. J., \& Marsh, H. W. (2013). Designing instructional text in a conversational style: A meta-analysis. Educational Psychology Review, 25(4), 445-472. http://doi.org/10.1007/s10648-013-9228-0

Goodboy, A. K., \& Bolkan, S. (2009). College teacher misbehaviors: Direct and indirect effects on student communication behavior and traditional learning outcomes. Western Journal of Communication, 73(2), 204-219. https://doi.org/10.1080/10570310902856089

Goodboy, A. K., Bolkan, S., \& Baker, J. P. (2018). Instructor misbehaviors impede students’ cognitive learning: Testing the causal assumption. Communication Education, 67(3), 308-329. http://doi.org/10.1080/03634523.2018.1465192

Goodboy, A. K., \& Myers, S. A. (2015). Revisiting instructor misbehaviors: A revised typology and development of a measure. Communication Education, 64(2), 133-153. 
http://doi.org/10.1080/03634523.2014.978798

Gorham, J. (1988). The relationship between verbal teacher immediacy behaviors and student learning. Communication Education, 37(1), 40-53. http://doi.org/10.1080/03634528809378702

Guo, P. J., Kim, J. \& Rubin, R. (2014). How video production affects student engagement: An empirical study of MOOC videos. In Proceedings of the first ACM conference on Learning (pp. 41-50). ACM Press. https://doi.org/10.1145/2556325.2566239

Harp, S. F., \& Mayer, R. E. (1997). The role of interest in learning from scientific text and illustrations: On the distinction between emotional interest and cognitive interest. Journal of Educational Psychology, 89(1), 92-102. http://doi.org/10.1037/0022-0663.89.1.92

Harp, S. F., \& Mayer, R. E. (1998). How seductive details do their damage: A theory of cognitive interest in science learning. Journal of Educational Psychology, 90(3), 414434. http://doi.org/10.1037/0022-0663.90.3.414

Harskamp, E. G., Mayer, R. E., \& Suhre, C. (2007). Does the modality principle for multimedia learning apply to science classrooms? Learning and Instruction, 17(5), 465-477. http://doi.org/10.1016/j.learninstruc.2007.09.010

Hart, S. G., \& Staveland, L. E. (1988). Development of NASA-TLX (Task Load Index): Results of empirical and theoretical research. Advances in Psychology, 52(1), 139183. https://doi.org/10.1016/S0166-4115(08)62386-9

Hasler, B. S., Kersten, B., \& Sweller, J. (2007). Learner control, cognitive load and instructional animation. Applied Cognitive Psychology, 21(6), 713-729. http://doi.org/10.1002/acp.1345

Hassanabadi, H., Robatjazi, E. S., \& Savoji, A. P. (2011). Cognitive consequences of 
segmentation and modality methods in learning from instructional animations. Procedia - Social and Behavioral Sciences, 30(1), 1481-1487.

http://doi.org/10.1016/j.sbspro.2011.10.287

Hayes, A. F. (2015). An index and test of linear moderated mediation. Multivariate Behavioral Research, 50(1), 1-22. https://doi.org/10.1080/00273171.2014.962683

Hayes, A. F. (2018). Introduction to mediation, moderation, and conditional process analysis: A regression-based approach ( $2^{\text {nd }}$ ed.). Guilford Press.

Hayes, A. F., \& Coutts, J. J. (2020). Use omega rather than Chronbach's alpha for estimating reliability. But.... Communication Methods and Measures, 14(1), 1-24. https://doi.org/10.1080/19312458.2020.1718629

Hayes, A. F., \& Preacher, K. J. (2014). Statistical mediation analysis with a multicategorical independent variable. British Journal of Mathematical and Statistical Psychology, 67(3), 451-470. https://doi.org/10.1111/bmsp.12028

Hess, A. (2020, July 27). More than $93 \%$ of U.S. college students say tuition should be lowered if classes are online. $C N B C$. https://www.cnbc.com/2020/07/27/93percent-of-collegestudents-say-tuition-should-be-cut-for-online-classes.html

Hew, K. F., Cheung, W. S., \& Ng, C. S. L. (2010). Student contribution in asynchronous online discussion: A review of the research and empirical exploration. Instructional Science, 38(6), 571-606. https://doi.org/10.1007/s11251-008-9087-0

Hewitt, J. (2005). Toward an understanding of how threads die in asynchronous computer conferences. Journal of Learning Sciences, 14(4), 567-589. https://doi.org/10.1207/s15327809j1s1404_4

Hidi, S., \& Baird, W. (1986). Interestingness - a neglected variable in discourse 
processing. Cognitive Science, 10(2), 179-194.

http://doi.org/10.1207/s15516709cog1002_3

Hidi, S., \& Baird, W. (1988). Strategies for increasing text-based interest and students' recall of expository texts. Reading Research Quarterly, 23(4), 465-483. http://doi.org/10.2307/747644

Hines, C. V., Cruickshank, D. R., \& Kennedy, J. J. (1985). Teacher clarity and its relationship to student achievement and satisfaction. American Educational Research Journal, 22(1), 87-99. http://doi.org/10.2307/1162989

Huber, S. G., \& Helm, C. (2020). COVID-19 and schooling: Evaluation, assessment, and accountability in times of crises - reacting quickly to explore key issues for policy, practice, and research with the school barometer. Educational Assessment, Evaluation, and Accountability, 32(1), 237-270. http://doi.org/10.1007/s11092-020-09322-y

Hubler, S. (2020, August 15). As colleges move classes online, families rebel against the cost. The New York Times. https://www.nytimes.com/2020/08/15/us/covid-college-tuition.html

Ibrahim, M., Antonenko, P. D., Greenwood, C. M., \& Wheeler, D. (2012). Effects of segmenting, signaling, and weeding on learning from educational video. Learning, Media, and Technology, 37(3), 220-235. http://doi.org/10.1080/17439884.2011.585993

Jamet, E., \& Le Bohec, O. (2007). The effect of redundant text in multimedia instruction. Contemporary Educational Psychology, 32(4), 588-598. http://doi.org/10.1016/j.cedpsych.2006.07.001

Jeung, H. J., Chandler, P., \& Sweller, J. (1997). The role of visual indicators in dual sensory mode instruction. Educational Psychology, 17(3), 329-343. http://doi.org/10.1080/0144341970170307 
Johnson, C. I., \& Mayer, R. E. (2009). A testing effect with multimedia learning. Journal of Educational Psychology, 101(3), 621-629. http://doi.org/10.1037/a0015183

Johnson, C. I., \& Mayer, R. E. (2010). Applying the self-explanation principle to multimedia learning in a computer-based game-like environment. Computers in Human Behavior, 26(6), 1246-1252. http://doi.org/10.1016/j.chb.2010.03.025

Johnson, C. I., \& Mayer, R. E. (2012). An eye movement analysis of the spatial contiguity effect in multimedia learning. Journal of Experimental Psychology: Applied, 18(2), 178-191. http://doi.org/10.1037/a0026923

Jones, L. C., \& Plass, J. L. (2002). Supporting listening comprehension and vocabulary acquisition in French with multimedia annotations. Modern Language Journal, 86(4), 546-561. http://doi.org/10.1111/1540-4781.00160

Jones, M. (2020, July 30). We've had a worldwide experiment in videoconferencing - an interview with Zoom. TechwireAsia. https://techwireasia.com/2020/07/weve-had-aworldwide-experiment-in-videoconferencing-an-interview-with-zoom/

Kali, Y., Goodyear, P., \& Markauskaite, L. (2011). Researching design practices and design cognition: Contexts, experiences, and pedagogical knowledge-in-pieces. Learning, Media, and Technology, 36 (2), 129-149. http://doi.org/10.1080/17439884.2011.553621

Kalyuga, S., Chandler, P., \& Sweller, J. (1998). Levels of expertise and instructional design. Human Factors, 40(1), 1-17. http://doi.org/10.1518/001872098779480587

Kalyuga, S., Chandler, P., \& Sweller, J. (1999). Managing split-attention and redundancy in multimedia instruction. Applied Cognitive Psychology, 13(4), 351-371. http://doi.org/10.1002/(SICI)1099-0720(199908)13:4<351::AID-ACP589>3.0.CO;2-6

Kalyuga, S., Chandler, P., \& Sweller, J. (2000). Incorporating learner experience into the design 
of multimedia instruction. Journal of Educational Psychology, 92(1), 126-136. http://doi.org/10.1037/0022-0663.92.1.126

Kartal, G. (2010). Does language matter in multimedia learning? Personalization principle revisited. Journal of Educational Psychology, 102(3), 615-624. http://doi.org/10.1037/a0019345

Kearney, P., Plax, T. G., Hays, E. R., \& Ivey, M. J. (1991). College teacher misbehaviors: What students don't like about what teachers say and do. Communication Quarterly, 39(4), 309-324. https://doi.org/10.1080/01463379109369808

Kennedy, J. J., Cruickshank, D. R., Bush, A. J., \& Myers, B. (1978). Additional investigations into the nature of teacher clarity. The Journal of Educational Research, 72(1), 3-10. http://doi.org/10.1080/00220671.1978.10885109

Kester, L., Kirschner, P. A., \& van Merriënboer, J. J. G. (2005). The management of cognitive load during complex cognitive skill acquisition by means of computer-simulated problem solving. British Journal of Educational Psychology, 75(1), 71-85. http://doi.org/10.1348/000709904X19254

Kester, L., Kirschner, P. A., \& van Merriënboer, J. J. G. (2006). Just-in-time information presentation: Improving learning a troubleshooting skill. Contemporary Educational Psychology, 31(2), 167-185. http://doi.org/10.1016/j.cedpsych.2005.04.002

Kline, T. J. B. (2005). Psychological testing: A practical approach to design and evaluation. SAGE. https://dx.doi.org/10.4135/9781483385693

Knoster, K. C., \& Goodboy, A. K. (2021). Making content relevant: A teaching and learning experiment with replication. Communication Education, 70(1), 4-26.

https://doi.org/10.1080/03634523.2020.1788106 
Korbach, A., Brünken, R., \& Park, B. (2016). Learner characteristics and information processing in multimedia learning: A moderated mediation of the seductive details effect. Learning and Individual Differences, 51(1), 59-68. http://doi.org/10.1016/j.lindif.2016.08.030

Kristóf, Z. (2020). International trends of remote teaching ordered in light of the Coronavirus (COVID-19) and its most popular video conferencing applications that implement communication. Central European Journal of Educational Research, 2(2), 84-92. http://doi.org/10.37441/CEJER/2020/2/2/7917

Kuznekoff, J. H. (2020). Online video lectures: The relationship between student viewing behaviors, learning, and engagement. Association for University Regional Campuses of Ohio Journal, 26, 33-55.

https://www.aurco.org/Journals/AURCO_Journal_2020/Harrison_Online_Video_2020.p df

LaBelle, S., Martin, M. M., \& Weber, K. (2013). Instructional dissent in the college classroom: Using the Instructional Beliefs Model as a framework. Communication Education, 62(2), 169-190. https://doi.org/10.1080/03634523.2012.759243

Land, M. L. (1979). Low-inference variables of teacher clarity: Effects on student concept learning. Journal of Educational Psychology, 71(6), 795-799. http://doi.org/10.1037/0022-0663.71.6.795

Land, M. L. (1981). Combined effect of two teacher clarity variables on student achievement. The Journal of Experimental Education, 50(1), 14-17. http://doi.org/10.1080/00220973.1981.11011794

Lange, C., Costley, J., \& Han, S. (2017). The effects of extraneous load on the relationship between self-regulated effort and germane load within an e-learning environment. 
International Review of Research in Open and Distributed Learning, 18(5), 64-83. https://doi.org/10.19173/irrodl.v18i5.3028

Leahy, W., Chandler, P., \& Sweller, J. (2003). When auditory presentations should and should not be a component of multimedia instruction. Applied Cognitive Psychology, 17(4), 401-418. http://doi.org/10.1002/acp.877

Leahy, W., \& Sweller, J. (2011). Cognitive load theory, modality of presentation and the transient information effect. Applied Cognitive Psychology, 25(6), 943-951. http://doi.org/10.1002/acp.1787

Lee, H., \& Mayer, R. E. (2015). Visual aids to learning in a second language: Adding redundant video to an audio lecture. Applied Cognitive Psychology, 29(3), 445-454. http://doi.org/10.1002/acp.3123

Lee, H., \& Mayer, R. E. (2018). Fostering learning from instructional video in a second language. Applied Cognitive Psychology, 32(5), 648-654. http://doi.org/10.1002/acp.3436

Lee, H., Plass, J. L., \& Homer, B. D. (2006). Optimizing cognitive load for learning from computer-based science simulations. Journal of Educational Psychology, 98(4), 902-913. http://doi.org/10.1037/0022-0663.98.4.902

Leopold, C., \& Mayer, R. E. (2015). An imagination effect in learning from scientific text. Journal of Educational Psychology, 107(1), 47-63. http://doi.org/10.1037/a0037142

Leopold, C., Mayer, R. E., \& Dutke, S. (2019). The power of imagination and perspective in learning from science text. Journal of Educational Psychology, 111(5), 793-808. http://doi.org/10.1037/edu0000310

Li, L., Ginns, P., Wang, T., \& Zhang, P. (2020). Using a pedagogical agent to deliver conversational style instruction: What benefits can you obtain? Computers \& Education, 
143(1), 1-11. http://doi.org/10.1016/j.compedu.2019.103658

Li, W., Wang, F., Mayer, R. E., \& Liu, H. (2019). Getting the point: Which kinds of gestures by pedagogical agents improve multimedia learning? Journal of Educational Psychology, 111(8), 1382-1395. http://doi.org/10.1037/edu0000352

Loman, N. L., \& Mayer, R. E. (1983). Signaling techniques that increase the understandability of expository prose. Journal of Educational Psychology, 75(3), 402-412. http://doi.org/10.1037/0022-0663.75.3.402

Low, R., \& Sweller, J. (2014). The modality principle in multimedia learning. In R. E. Mayer (Ed.), The Cambridge handbook of multimedia learning ( $2^{\text {nd }}$ ed., pp. 227-246). Cambridge University Press. https://doi.org/10.1017/cbo9781139547369.012

Lusk, M. M., \& Atkinson, R. K. (2007). Animated pedagogical agents: Does their degree of embodiment impact learning from static or animated work examples? Applied Cognitive Psychology, 21(6), 747-764. http://doi.org/10.1002/acp.1347

Makransky, G., Borre-Gude, S., \& Mayer, R. E. (2019). Motivational and cognitive benefits of training in immersive virtual reality based on multiple assessments. Journal of Computer Assisted Learning, 35(6), 691-707. http://doi.org/10.1111/jcal.12375

Makransky, G., Terkildsen, T. S., \& Mayer, R. E. (2019a). Adding immersive virtual reality to a science lab simulation causes more presence but less learning. Learning and Instruction, 60 (1), 225-236. http://doi.org/10.1016/j.learninstruc.2017.12.007

Makransky, G., Terkildsen, T. S., \& Mayer, R. E. (2019b). Role of subjective and objective measures of cognitive processing during learning in explaining the spatial contiguity effect. Learning and Instruction, 61(1), 23-34. http://doi.org/10.1016/j.learninstruc.2018.12.001 
Mautone, P. D., \& Mayer, R. E. (2001). Signaling as a cognitive guide in multimedia learning. Journal of Educational Psychology, 93(2), 377-389. http://doi.org/10.1037/0022-0663.93.2.377

Mautone, P. D., \& Mayer, R. E. (2007). Cognitive aids for guiding graph comprehension. Journal of Educational Psychology, 99(3), 640-652. http://doi.org/10.1037/0022-0663.99.3.640

Mayer, R. E. (1975). Forward transfer of different reading strategies evoked by testlike events in mathematics text. Journal of Educational Psychology, 67(2), 165-169. http://doi.org/10.1037/h0077011

Mayer, R. E. (1976). Some conditions of meaningful learning for computer programming: Advance organizers and subject control of frame order. Journal of Educational Psychology, 68(2), 143-150. http://doi.org/10.1037/0022-0663.68.2.143

Mayer, R. E. (1979a). Can advance organizers influence meaningful learning? Review of Educational Research, 49(2), 371-383. http://doi.org/10.2307/1169964

Mayer, R. E. (1979b). Twenty years of research on advance organizers: Assimilation theory is still the best predictor of results. Instructional Science, 8(2), 133-167. http://doi.org/10.1007/BF00117008

Mayer, R. E. (1980). Elaboration techniques that increase the meaningfulness of technical text: An experimental test of the learning strategy hypothesis. Journal of Educational Psychology, 72(6), 770-784. http://doi.org/10.1037/0022-0663.72.6.770

Mayer, R. E. (1983). Can you repeat that? Qualitative effects of repetition and advance organizers on learning from science prose. Journal of Educational Psychology, 75(1), 40-49. http://doi.org/10.1037/0022-0663.75.1.40 
Mayer, R. E. (1989). Systematic thinking fostered by illustrations in scientific text. Journal of Educational Psychology, 81(2), 240-246. http://doi.org/10.1037/0022-0663.81.2.240

Mayer, R. E. (2001). Multimedia learning. Cambridge University Press. https://doi.org/10.1017/CBO9781139164603

Mayer, R. E. (2005a). Cognitive theory of multimedia learning. In R. E. Mayer (Ed.), The Cambridge handbook of multimedia learning ( $1^{\text {st }}$ ed., pp. 31- 48). Cambridge University Press. https://doi.org/10.1017/cbo9780511816819.004

Mayer, R. E. (2005b). Principles for managing essential processing in multimedia learning: Segmenting, pretraining, and modality principles. In R. E. Mayer (Ed.), The Cambridge handbook of multimedia learning ( $1^{\text {st }}$ ed., pp. 169-182). Cambridge University Press. https://doi.org/10.1017/cbo9780511816819.012

Mayer, R. E. (2005c). Principles for reducing extraneous processing in multimedia learning: Coherence, signaling, redundancy, spatial contiguity, and temporal contiguity principles. In R. E. Mayer (Ed.), The Cambridge handbook of multimedia learning ( $1^{\text {st }}$ ed., pp. 183200). Cambridge University Press. https://doi.org/10.1017/cbo9780511816819.013

Mayer, R. E. (2005d). Principles of multimedia learning based on social cues: Personalization, voice, and image principles. In R. E. Mayer (Ed.), The Cambridge handbook of multimedia learning ( $1^{\text {st }}$ ed., pp. 201-214). Cambridge University Press. https://doi.org/10.1017/cbo9780511816819.014

Mayer, R. E. (2011). Applying the science of learning to multimedia instruction. In J. P. Mestre \& B. H. Ross (Eds.), The psychology of learning and motivation: Volume 55 (p. 77-108). Elsevier Academic Press. https://doi.org/10.1016/b978-0-12-387691-1.00003-x Mayer, R. E. (2014a). Cognitive theory of multimedia learning. In R. E. Mayer (Ed.), The 
Cambridge handbook of multimedia learning ( $2^{\text {nd }}$ ed., pp. 43-71). Cambridge University Press. https://doi/10.1017/CBO9781139547369.005

Mayer, R. E. (2014b). Incorporating motivation into multimedia learning. Learning and Instruction, 29(1), 171-173. https://doi.org/10.1016/j.learninstruc.2013.04.003

Mayer, R. E. (2021). Multimedia learning ( $3^{\text {rd }}$ ed.). New York, NY: Cambridge University Press. https://doi.org/10.1017/9781316941355

Mayer, R. E., \& Anderson, R. B. (1991). Animations need narrations: An experimental test of a dual-coding hypothesis. Journal of Educational Psychology, 83(4), 484-490. http://doi.org/10.1037/0022-0663.83.4.484

Mayer, R. E., \& Anderson, R. B. (1992). The instructive animation: Helping students build connections between words and pictures in multimedia learning. Journal of Educational Psychology, 84(4), 444-452. http://doi.org/10.1037/0022-0663.84.4.444

Mayer, R. E., Bove, W., Bryman, A., Mars, R., \& Tapangco, L. (1996). When less is more: Meaningful learning from visual and verbal summaries of science textbook lessons. Journal of Educational Psychology, 88(1), 64-73. http://doi.org/10.1037/0022-0663.88.1.64

Mayer, R. E., Brandt, D. M., \& Bluth, G. J. (1980). Use of top-level structure in text: Key for reading comprehension of ninth-grade students. Reading Research Quarterly, 16(1), 72103. http://doi.org/10.2307/747349

Mayer, R. E., \& Chandler, P. (2001). When learning is just a click away: Does simple user interaction foster deeper understanding of multimedia messages? Journal of Educational Psychology, 93(2), 390-397. http://doi.org/10.1037/0022-0663.93.2.390

Mayer, R. E., \& DaPra, C. S. (2012). An embodiment effect in computer-based learning with 
animated pedagogical agents. Journal of Experimental Psychology: Applied, 18(3), 239252. http://doi.org/10.1037/a0028616

Mayer, R. E., Dow, G. T., \& Mayer, S. (2003). Multimedia learning in an interactive selfexplaining environment: What works in the design of agent-based microworlds? Journal of Educational Psychology, 95(4), 806-812. http://doi.org/10.1037/0022-0663.95.4.806

Mayer, R. E., Dyck, J. L., \& Cook, L. K. (1984). Techniques that help readers build mental models from scientific text: Definitions pretraining and signaling. Journal of Educational Psychology, 76(6), 1089-1105. http://doi.org/10.1037/0022-0663.76.6.1089

Mayer, R. E., \& Estrella, G. (2014). Benefits of emotional design in multimedia instruction. Learning and Instruction, 33(1), 12-18. https://doi.org/10.1016/j.learninstruc.2014.02.004

Mayer, R. E., Fennell, S., Farmer, L., \& Campbell, J. (2004). A personalization effect in multimedia learning: Students learn better when words are in conversational style rather than formal style. Journal of Educational Psychology, 96(2), 389-395. http://doi.org/10.1037/0022-0663.96.2.389

Mayer, R. E., \& Fiorella, L. (2014). Principles for reducing extraneous processing in multimedia learning: Coherence, signaling, redundancy, spatial contiguity, and temporal contiguity principles. In R. E. Mayer (Ed.), The Cambridge handbook of multimedia learning $\left(2^{\text {nd }}\right.$ ed., pp. 279-315). Cambridge University Press. https://doi.org/10.1017/cbo9781139547369.015

Mayer, R. E., \& Gallini, J. K. (1990). When is an illustration worth ten thousand words? Journal of Educational Psychology, 82(4), 715-726. http://doi.org/10.1037/0022-0663.82.4.715

Mayer, R. E., Griffith, E., Jurkowitz, I. T. N., \& Rothman, D. (2008). Increased interestingness 
of extraneous details in a multimedia science presentation leads to decreased learning. Journal of Experimental Psychology: Applied, 14(4), 329-339. http://doi.org/10.1037/a0013835

Mayer, R. E., Heiser, J., \& Lonn, S. (2001). Cognitive constraints on multimedia learning: When presenting more material results in less understanding. Journal of Educational Psychology, 93(1), 187-198. http://doi.org/10.1037/0022-0663.93.1.187

Mayer, R. E., Howarth, J. T., Kaplan, M., \& Hanna, S. (2018). Applying the segmenting principle to online geography slideshow lessons. Educational Technology Research and Development, 66(3), 563-577. http://doi.org/10.1007/s11423-017-9554-x

Mayer, R. E., \& Jackson, J. (2005). The case for coherence in scientific explanations: Quantitative details can hurt qualitative understanding. Journal of Experimental Psychology: Applied, 11(1), 13-18. http://doi.org/10.1037/1076-898X.11.1.13

Mayer, R. E., \& Johnson, C. I. (2008). Revising the redundancy principle in multimedia learning. Journal of Educational Psychology, 100(2), 380-386. http://doi.org/10.1037/0022-0663.100.2.380

Mayer, R. E., \& Johnson, C. I. (2010). Adding instructional features that promote learning in a game-like environment. Journal of Educational Computing Research, 42(3), 241-265. http://doi.org/10.2190/EC.42.3.a

Mayer, R. E., Lee, H., \& Peebles, A. (2014). Multimedia learning in a second language: A cognitive load perspective. Applied Cognitive Psychology, 28(5), 653-660. http://doi.org/10.1002/acp.3050

Mayer, R. E., Mathias, A., \& Wetzell, K. (2002a). Fostering understanding of multimedia messages through pre-training: Evidence for a two-stage theory of mental model 
construction. Journal of Experimental Psychology: Applied, 8(3), 147-154.

http://doi.org/10.1037/1076-898X.8.3.147

Mayer, R. E., Mautone, P., \& Prothero, W. (2002b). Pictorial aids for learning by doing in a multimedia geology simulation game. Journal of Educational Psychology, 94(1), 171185. http://doi.org/10.1037/0022-0663.94.1.171

Mayer, R. E., \& Moreno, R. (1998). A split-attention effect in multimedia learning: Evidence for dual processing systems in working memory. Journal of Educational Psychology, 90(2), 312-320. http://doi.org/10.1037/0022-0663.90.2.312

Mayer, R. E., \& Moreno, R. (2003). Nine ways to reduce cognitive load in multimedia learning. Educational Psychologist, 38(1), 43-52. http://doi.org/10.1207/S15326985EP3801_6

Mayer, R. E., Moreno, R., Boire, M., \& Vagge, S. (1999). Maximizing constructivist learning from multimedia communications by minimizing cognitive load. Journal of Educational Psychology, 91(4), 638-643. http://doi.org/10.1037/0022-0663.91.4.638

Mayer, R. E., \& Pilegard, C. (2014). Principles for managing essential processing in multimedia learning: Segmenting, pre-training, and modality principles. In R. E. Mayer (Ed.), The Cambridge handbook of multimedia learning (2 ${ }^{\text {nd }}$ ed., pp. 316-344). Cambridge University Press. https://doi.org/10.1017/cbo9781139547369.016

Mayer, R. E., \& Sims, V. K. (1994). For whom is a picture worth a thousand words? Extensions of a dual-coding theory of multimedia learning. Journal of Educational Psychology, 86(3), 389-401. http://doi.org/10.1037/0022-0663.86.3.389

Mayer, R. E., Sobko, K., \& Mautone, P. D. (2003). Social cues in multimedia learning: Role of speaker's voice. Journal of Educational Psychology, 95(2), 419-425. 
http://doi.org/10.1037/0022-0663.95.2.419

Mayer, R. E., Steinhoff, K., Bower, G., \& Mars, R. (1995). A generative theory of textbook design: Using annotated illustrations to foster meaningful learning of science text. Educational Technology Research and Development, 43(1), 31-43. http://doi.org/10.1007/BF02300480

Mayer, R. E., Wells, A., Parong, J., \& Howarth, J. T. (2019). Learning control of the pacing of an online slideshow lesson: Does segmenting help? Applied Cognitive Psychology, 33(5), 930-935. http://doi.org/10.1002/acp.3560

Mazer, J. P. (2012). Associations among teacher communication behaviors, student interest, and engagement: A validity test. Communication Education, 62(1), 86-96. https://doi.org/10.1080/03634523.2012.731513

Mazer, J. P. (2018). Instructor message variables. In M. L. Houser \& A. M. Hosek (Eds.), Handbook of instructional communication: Rhetorical and relational perspectives $\left(2^{\text {nd }}\right.$ ed., pp. 22-37). Routledge. https://doi.org/10.4324/9781315189864

Mazer, J. P., \& Graham, E. E. (2019). Measurement in instructional communication. In J. P. Mazer \& E. E. Graham (Eds.), Communication research measures II: A sourcebook (pp. 54-69). Routledge. https://doi.org/10.4324/9780203730188

Mazer, J. P., McKenna-Buchanan, T. P., Quinlan, M. M., \& Titsworth, S. (2014). The dark side of emotion in the classroom: Emotional processes as mediators of teacher communication behaviors and student negative emotions. Communication Education, 63(3), 149-168. https://doi.org/10.1080/03634523.2014.904047

McBrien, J. L., Cheng, R., \& Jones, P. (2020). Virtual spaces: Employing a synchronous online classroom to facilitate student engagement in online learning. The International Review 
of Research in Open and Distributed Learning, 10(3), 1-17. https://doi.org/10.19173/irrodl.v10i3.605

McCroskey, J. C. (1994). Assessment of affect toward communication and affect toward instruction in communication. In S. Morreale \& M. Brooks (Eds.), Assessing college student competency in communication (pp. 56-70). Speech Communication Association.

McCrudden, M. T., Magliano, J. P., \& Schraw, G. (2011). The effect of diagrams on online reading processes and memory. Discourse Processes, 48(2), 69-92. http://doi.org/10.1080/01638531003694561

McLaren, B. M., DeLeeuw, K. E., \& Mayer, R. E. (2011a). A politeness effect in learning with web-based intelligent tutors. International Journal of Human-Computer Studies, 69(1-2), 70-79. http://doi.org/10.1016/j.ijhcs.2010.09.001

McLaren, B. M., DeLeeuw, K. E., \& Mayer, R. E. (2011b). Polite web-based intelligent tutors: Can they improve learning in classrooms? Computers \& Education, 56(3), 574-584. http://doi.org/10.1016/j.compedu.2010.09.019

Means, B., \& Neisler, J. (2020). Suddenly online: A national survey of undergraduates during the COVID-19 pandemic. Digital Promise.

Michas, I. C., \& Berry, D. C. (2000). Learning a procedural task: Effectiveness of multimedia presentations. Applied Cognitive Psychology, 14(6), 555-575. http://doi.org/10.1002/1099-0720(200011/12)14:6<555::AID-ACP677>3.0.CO;2-4

Moreno, R. (2005). Instructional technology: Promises and pitfalls. In L. PytlikZillig, M. Bodvarsson, \& R. Bruning (Eds.), Technology-based education: Bringing researchers and practitioners together (pp. 1-19). Greenwich, CT: Information Age Publishing.

Moreno, R. (2006). Does the modality principle hold for different media? A test of the method- 
affects-learning hypothesis. Journal of Computer Assisted Learning, 22(3), 149-158. http://doi.org/10.1111/j.1365-2729.2006.00170.x

Moreno, R. (2007). Optimising learning from animations by minimizing cognitive load:

Cognitive and affective consequences of signaling and segmentation methods. Applied Cognitive Psychology, 21(6), 765-781. http://doi.org/10.1002/acp.1348

Moreno, R., \& Mayer, R. E. (1999a). Cognitive principles of multimedia learning: The role of modality and contiguity. Journal of Educational Psychology, 91(2), 358-368. http://doi.org/10.1037/0022-0663.91.2.358

Moreno, R., \& Mayer, R. E. (1999b). Multimedia-supported metaphors for meaning making in mathematics. Cognition and Instruction, 17(3), 215-248. http://doi.org/10.1207/S1532690XCI1703_1

Moreno, R., \& Mayer, R. E. (2000). Engaging students in active learning: The case for personalized multimedia messages. Journal of Educational Psychology, 92(4), 724-733. http://doi.org/10.1037/0022-0663.92.4.724

Moreno, R., \& Mayer, R. E. (2002a). Verbal redundancy in multimedia learning: When reading helps listening. Journal of Educational Psychology, 94(1), 156-163. http://doi.org/10.1037/0022-0663.94.1.156

Moreno, R., \& Mayer, R. E. (2002b). Learning science in virtual reality multimedia environments: Role of methods and media. Journal of Educational Psychology, 94(3), 598-610. http://doi.org/10.1037/0022-0663.94.3.598

Moreno, R., \& Mayer, R. E. (2004). Personalized messages that promote science learning in virtual environments. Journal of Educational Psychology, 96(1), 165-173. http://doi.org/10.1037/0022-0663.96.1.165 
Moreno, R., Mayer, R. E., Spires, H. A., \& Lester, J. C. (2001). The case for social agency in computer-based teaching: Do students learn more deeply when they interact with animated pedagogical agents? Cognition and Instruction, 19(2), 177-213. http://doi.org/10.1207/S1532690XCI1902_02

Moreno, R., Reislein, M., \& Ozogul, G. (2010). Using virtual peers to guide visual attention during learning: A test of the persona hypothesis. Journal of Media Psychology: Theories, Methods, and Applications, 22(2), 52-60. http://doi.org/10.1027/1864-1105/a000008

Mottet, T. P. (2015). Affective learning from a cognitive neuroscientific perspective. Communication Education, 64(4), 508-510. https://doi.org/10.1080/03634523.2015.1064144

Mottet, T. P., \& Beebe, S. A. (2006). Foundations of instructional communication. In T. P. Mottet, V. P. Richmond, \& J. C. McCroskey (Eds.), Handbook of instructional communication: Rhetorical and relational perspectives (pp. 3-32). Allyn \& Bacon. https://doi.org/10.4324/9781315664064

Mottet, T. P., Frymier, A. B., \& Beebe, S. A. (2006). Theorizing about instructional communication. In T. P. Mottet, V. P. Richmond, \& J. C. McCroskey (Eds.), Handbook of instructional communication: Rhetorical and relational perspectives (pp. 255-282). Allyn \& Bacon. https://doi.org/10.4324/9781315664064

Mousavi, S. Y., Low, R., \& Sweller, J. (1995). Reducing cognitive load by mixing auditory and visual presentation modes. Journal of Educational Psychology, 87(2), 319334. http://doi.org/10.1037/0022-0663.87.2.319

Murphy, M. P. A. (2020). COVID-19 and emergency elearning: Consequences of the 
securitization of higher education for post-pandemic pedagogy. Contemporary Security Policy, 41(3), 492-505. http://doi.org/10.1080/13523260.2020.1761749

Muthén, L. K., \& Muthén, B. O. (2002). How to use a Monte Carlo study to decide on sample size and determine power. Structural Equation Modeling: A Multidisciplinary Journal, 9(4), 599-620. https://doi.org/10.1207/S15328007SEM0904_8

Naumann, J., Richter, T., Flender, J., Christmann, U., \& Groeben, N. (2007). Signaling in expository hypertexts compensates for deficits in reading skill. Journal of Educational Psychology, 99(4), 791-807. http://doi.org/10.1037/0022-0663.99.4.791

Offir, B., Lev, Y., \& Bezalel, R. (2008). Surface and deep learning processes in distance education: Synchronous versus asynchronous systems. Computers \& Education, 51(3), 1172-1183. https://doi.org/10.1016/j.compedu.2007.10.009

Paas, F., \& Sweller, J. (2014). Implications of cognitive load theory for multimedia learning, in R. E. Mayer (Ed.), The Cambridge handbook of multimedia learning (2 $2^{\text {nd }}$ ed., pp. 27- 42). Cambridge University Press. https://doi.org/10.1017/cbo9781139547369.004

Paas, F., Tuovinen, J. E., Tabbers, H., van Gerven, P. W. M. (2003). Cognitive load measurement as a means to advance cognitive load theory. Educational Psychologist, 38(1), 63-71. http://doi.org/10.1207/S15326985EP3801_8

Paas, F. G. W. C., \& van Merriënboer, J. J. G. (1994). Instructional control of cognitive load in the training of complex cognitive tasks. Educational Psychology Review, 6(1), 351-371. http://doi.org/10.1007/BF02213420

Paivio, A. (1965). Abstractness, imagery, and meaningfulness in paired-associate learning. Journal of Verbal Learning and Verbal Behavior, 4(1), 32-38. http://doi.org/10.1016/S0022-5371(65)80064-0 
Paivio, A. (1986). Mental representations: A dual coding approach. Oxford University Press. https://doi.org/10.1093/acprof:oso/9780195066661.001.0001

Paivio, A. (2006). Dual coding theory and education. In S. Neuman (ed.), Pathways to literacy achievement for high poverty children. University of Michigan. https://doi.org/10.1007/BF01320076

Park, S. (2015). The effects of social cue principles on cognitive load, situational interest, motivation, and achievement in pedagogical agent multimedia learning. Journal of Educational Technology \& Society, 18(1), 211-229. https://eric.ed.gov/?id=EJ1078321

Parong, J., \& Mayer, R. E. (2018). Learning science in immersive virtual reality. Journal of Educational Psychology, 110(6), 785-797. http://doi.org/10.1037/edu0000241

Penney, C. G. (1989). Modality effects and the structure of short-term verbal memory. Memory \& Cognition, 17(4), 398-422. http://doi.org/10.3758/bf03202613

Peper, R. J., \& Mayer, R. E. (1978). Note taking as a generative activity. Journal of Educational Psychology, 70(4), 514-522. http://doi.org/10.1037/0022-0663.70.4.514

Peper, R. J., \& Mayer, R. E. (1986). Generative effects of note-taking during science lectures. Journal of Educational Psychology, 78(1), 34-38. http://doi.org/10.1037/0022-0663.78.1.34

Peterson, A. T., Beymer, P. N., \& Putnam, R. T. (2018). Synchronous and asynchronous discussions: Effects on cooperation, belonging, and affect. Online Learning, 22(4), 7-25. http://doi.org/10.24059/olj.v22i4.1517

Pilegard, C., \& Mayer, R. E. (2016). Improving academic learning from computer-based narrative games. Contemporary Educational Psychology, 44-45, 12-20. http://doi.org/10.1016/j.cedpsych.2015.12.002 
Pintrich, P. R., Smith, D. A. F., Garcia, T., \& McKeachie, W. J. (1991). A manual for the use of the motivated strategies for learning questionnaire. Office of Educational Research and Improvement.

Plass, J. L., Chun, D. M., Mayer, R. E., \& Leutner, D. (1998). Supporting visual and verbal learning preferences in a second-language multimedia learning environment. Journal of Educational Psychology, 90(1), 25-36. http://doi.org/10.1037/0022-0663.90.1.25

Pociask, F. D., \& Morrison, G. R. (2008). Controlling split attention and redundancy in physical therapy instruction. Educational Technology Research and Development, 56(1), 379-399. http://doi.org/10.1007/s11423-007-9062-5

Pollock, E., Chandler, P., \& Sweller, J. (2002). Assimilating complex information. Learning and Instruction, 12(1), 61-86. http://doi.org/10.1016/S0959-4752(01)00016-0

Ponce, H. R., \& Mayer, R. E. (2014a). Qualitatively different cognitive processing during online reading primed by different study activities. Computers in Human Behavior, 30(1), 121130. http://doi.org/10.1016/j.chb.2013.07.054

Ponce, H. R., \& Mayer, R. E. (2014b). An eye movement analysis of highlighting and graphic organizer study aids for learning from expository text. Computers in Human Behavior, 41(1), 21-32. http://doi.org/10.1016/j.chb.2014.09.010

Ponce, H. R., Mayer, R. E., Loyola, M. S., López, M. J., \& Méndez, E. E. (2018). When two computer-supported learning strategies are better than one: An eye-tracking study. Computers \& Education, 125(1), 376-388. http://doi.org/10.1016/j.compedu.2018.06.024

Ramlatchan, M., \& Watson, G. S. (2020). Enhancing instructor credibility and immediacy in online multimedia designs. Educational Technology Research and Development, 68(1), 511-528. http://doi.org/10.1007/s11423-019-09714-y 
Rapanta, C., Botturi, L., Goodyear, P., Guàrdia, L., \& Koole, M. (2020). Online university teaching during and after the COVID-19 crisis: Refocusing teacher presence and learning activity. Postdigital Science and Education, 2(1), 923-945. http://doi.org/10.1007/s42438-020-00155-y

Reichelt, M., Kämmerer, F., Niegemann, H. M., \& Zander, S. (2014). Talk to me personally: Personalization of language style in computer-based learning. Computers in Human Behavior, 35(1), 199-210. http://doi.org/10.1016/j.chb.2014.03.005

Reinwein, J. (2012). Does the modality effect exist? And if so, which modality effect? Journal of Psycholinguistic Research, 41(1), 1-32. http://doi.org/10.1007/s10936-011-9180-4

Rey, G. D. (2014). Seductive details and attention distraction - An eye tracker experiment. Computers in Human Behavior, 32(1), 133-144. http://doi.org/10.1016/j.chb.2013.11.017

Rey, G. D., Beege, M., Nebel, S., Wirzberger, M., Schmitt, T. H., \& Schneider, S. (2019). A meta-analysis of the segmenting effect. Educational Psychology Review, 31(2), 389-419. http://doi.org/10.1007/s10648-018-9456-4

Rey, G. D., \& Steib, N. (2013). The personalization effect in multimedia learning: The influence of dialect. Computers in Human Behavior, 29(5), 2022-2028. http://doi.org/10.1016/j.chb.2013.04.003

Richardson, J. (2003). Dual coding versus relational processing in memory for concrete and abstract words. European Journal of Cognitive Psychology, 15(4), 481-509. http://doi.org/10.1080/09541440244000256

Richardson, M., Abraham, C., \& Bond, R. (2012). Psychological correlates of university students' academic performance: A systematic review and meta-analysis. Psychological 
Bulletin, 138(2), 353-387. http://doi.org/10.1037/a0026838

Richter, J., Scheiter, K., \& Eitel, A. (2016). Signaling text-picture relations in multimedia learning: A comprehensive meta-analysis. Educational Research Review, 17(1), 19-36. http://doi.org/10.1016/j.edurev.2015.12.003

Richter, J., Scheiter, K., \& Eitel, A. (2018). Signaling text-picture relations in multimedia learning: The influence of prior knowledge. Journal of Educational Psychology, 110(4), 544-560. https://doi.org/10.1037/edu0000220

Rocca, K. A. (2009). Participation in the college classroom: The impact of instructor immediacy and verbal aggression. Journal of Classroom Interaction, 43, 22-33. https://eric.ed.gov/?id=EJ859697

Rodríguez, J. I., Plax, T. G., \& Kearney, P. (1996). Clarifying the relationship between teacher nonverbal immediacy and student cognitive learning: Affective learning as the central causal mediator. Communication Education, 45(4), 293-305. http://doi.org/10.1080/03634529609379059

Sadoski, M. (1983). An exploratory study of the relationships between reported imagery and the comprehension and recall of a story. Reading Research Quarterly, 19(1), 110-123. http://doi.org/10.2307/747341

Sadoski, M. (1985). The natural use of imagery in story comprehension and recall: Replication and extension. Reading Research Quarterly, 20(5), 658-667. http://doi.org/10.2307/747949

Sagerman, N., \& Mayer, R. E. (1987). Forward transfer of different reading strategies evoked by adjunct questions in science text. Journal of Educational Psychology, 79(2), 189191. http://doi.org/10.1037/0022-0663.79.2.189 
Sanchez, C. A., \& Wiley, J. (2006). An examination of the seductive details effect in terms of working memory capacity. Memory \& Cognition, 34(2), 344-355. http://doi.org/10.3758/BF03193412

Schmidt-Weigand, F., Kohnert, A., \& Glowalla, U. (2010). Explaining the modality and contiguity effects: New insights from investigating students' viewing behaviour. Applied Cognitive Psychology, 24(2), 226-237. http://doi.org/10.1002/acp.1554

Schmeck, A., Mayer, R. E., Opfermann, M., Pfeiffer, V., \& Leutner, D. (2014). Drawing pictures during learning from scientific text: Testing the generative drawing effect and the prognostic drawing effect. Contemporary Educational Psychology, 39(4), 275-286. http://doi.org/10.1016/j.cedpsych.2014.07.003

Schneider, S., Beege, M., Nebel, S., \& Ray, G. D. (2018). A meta-analysis of how signaling affects learning with media. Educational Research Review, 23(1), 1-24. http://doi.org/10.1016/j.edurev.2017.11.001

Schwamborn, A., Mayer, R. E., Thillmann, H., Leopold, C., \& Leutner, D. (2010). Drawing as a generative activity and drawing as a prognostic activity. Journal of Educational Psychology, 102(4), 872-879. http://doi.org/10.1037/a0019640

Shrager, L., \& Mayer, R. E. (1989). Note-taking fosters generative learning strategies in novices. Journal of Educational Psychology, 81(2), 263-264. http://doi.org/10.1037/0022-0663.81.2.263

Schrader, C., Reichelt, M., \& Zander, S. (2018). The effect of the personalization principle on multimedia learning: The role of student individual interests as a predictor. Educational Technology Research \& Development, 66(6), 1387-1397.

http://doi.org/10.1007/s11423-018-9588-8 
Schworm, S., \& Stiller, K. D. (2012). Does personalization matter? The role of social cues in instructional explanations. Intelligent Decision Technology, 6(2), 105-111. http://doi.org/10.3233/IDT-2012-0127

Sitzmann, T., Ely, K., Brown, K. G., \& Bauer, K. N. (2010). Self-assessment of knowledge: A cognitive learning or affective measure? Academy of Management Learning \& Education, 9(2), 169-191. https://doi.org/10.5465/AMLE.2010.51428542

Spanjers, I. A. E., Wouters, P., van Gog, T., \& van Merriënboer, J. J. G. (2011). An expertise reversal effect of segmentation in learning from animated worked-out examples. Computers in Human Behavior, 27(1), 46-52. http://doi.org/10.1016/j.chb.2010.05.011

Stafford, V. (2020). EdTech review: Teaching through Zoom - what we've learned as new online educators. Journal of Applied Learning \& Teaching, 3(2), 1-4. http://doi.org/10.37074/jalt.2020.3.2.14

Stiller, K. D., Freitag, A., Zinnbauer, P. \& Freitag, C. (2009). How pacing of multimedia instructions can influence modality effects: A case of superiority of visual texts. Austraslasian Journal of Educational Technology, 25(2), 184-203. http://doi.org/10.14742/ajet.1149

Stiller, K. D., \& Jedlicka, R. (2010). A kind of expertise reversal effect: Personalisation effect can depend on domain-specific prior knowledge. Austraslian Journal of Educational Technology, 26(1), 133-149. http://doi.org/10.14742/ajet.1107

Stull, A. T., Fiorella, L., Gainer, M. J., \& Mayer, R. E. (2018). Using transparent whiteboards to boost learning from online STEM lectures. Computers \& Education, 120(1), 146-159. http://doi.org/10.1016/j.compedu.2018.02.005 
Stull, A. T., Fiorella, L., \& Mayer, R. E. (2018). An eye-tracking analysis of instructor presence in video lectures. Computers in Human Behavior, 88(1), 263-272. http://doi.org/10.1016/j.chb.2018.07.019

Stull, A. T., \& Mayer, R. E. (2007). Learning by doing versus learning by viewing: Three experimental comparisons of learner-generated versus author-provided graphic organizers. Journal of Educational Psychology, 99(4), 808-820. http://doi.org/10.1037/0022-0663.99.4.808

Sung, E., \& Mayer, R. E. (2012). When graphics improve liking but not learning from online lessons. Computers in Human Behavior, 28(5), 1618-1625. http://doi.org/10.1016/j.chb.2012.03.026

Sung, E., \& Mayer, R. E. (2013). Online multimedia learning with mobile devices and desktop computers: An experimental test of Clark's methods-not-media hypothesis. Computers in Human Behavior, 29(3), 639-647. http://doi.org/10.1016/j.chb.2012.10.022

Suskie, L. (2018). Assessing students learning: A common sense guide ( $3^{\text {rd }}$ ed.). Jossey-Bass.

Sweller, J. (1988). Cognitive load during problem solving: Effects on learning. Cognitive Science, 12(2), 257-285. http://doi.org/10.1016/0364-0213(88)90023-7

Sweller, J. (1994). Cognitive load theory, learning difficulty, and instructional design. Learning and Instruction, 4(4), 295-312. http://doi.org/10.1016/0959-4752(94)90003-5

Sweller, J. (2010). Element interactivity and intrinsic, extraneous, and germane cognitive load. Educational Psychology Review, 22(2), 123-138. http://doi.org/10.1007/s 10648-010-9128-5

Sweller, J., Ayres, P., \& Kalyuga, S. (2011). Cognitive load theory. Springer. https://doi.org/10.1007/978-1-4419-8126-4 
Sweller, J., \& Chandler, P. (1994). Why some material is difficult to learn. Cognition and Instruction, 12(3), 185-233. http://doi.org/10.1207/s1532690xci1203_1

Sweller, J., Chandler, P., Tierney, P., \& Cooper, M. (1990). Cognitive load as a factor in the structuring of technical material. Journal of Experimental Psychology: General, 119(2), 176-192. http://doi.org/10.1037/0096-3445.119.2.176

Tabbers, H. K., Martens, R. L., \& van Merriënboer, J. J. G. (2004). Multimedia instructions and cognitive load theory: Effects of modality and cueing. British Journal of Educational Psychology, 74(1), 71-81. http://doi.org/10.1348/000709904322848824

Thompson, V. A., \& Paivio, A. (1994). Memory for pictures and sounds: Independence of auditory and visual codes. Canadian Journal of Experimental Psychology, 48(3), 380398. http://doi.org/10.1037/1196-1961.48.3.380

Tindall-Ford, S., Chandler, P., \& Sweller, J. (1997). When two sensory modes are better than one. Journal of Experimental Psychology: Applied, 3(4), 257-287. http://doi.org/10.1037/1076-898X.3.4.257

Titsworth, B. S. (2001). The effects of teacher immediacy, use of organizational lecture cures, and students' notetaking on cognitive learning. Communication Education, 50(4), 283297. http://doi.org/10.1080/03634520109379256

Titsworth, B. S. (2004). Students' notetaking: The effects of teacher immediacy and clarity. Communication Education, 53(4), 305-320. http://doi.org/10.1080/0363452032000305922

Titsworth, S., Mazer, J. P., Goodboy, A. K., Bolkan, S., \& Myers, S. A. (2015). Two metaanalyses exploring the relationship between teacher clarity and student learning. Communication Education, 64(4), 385-418. 
http://doi.org/10.1080/03634523.2015.1041998

Titsworth, S., McKenna, T. P., Mazer, J. P., \& Quinlan, M. M. (2013). The bright side of emotion in the classroom: Do teachers' behaviors predict students' enjoyment, hope, and pride? Communication Education, 62(2), 191-209. https://doi.org/10.1080/03634523.2013.763997

Titsworth, B. S., \& Kiewra, K. A. (2004). Spoken organizational lecture cues and student notetaking as facilitators of student learning. Contemporary Educational Psychology, 29(4), 447-461. http://doi.org/10.1016/j.cedpsych.2003.12.001

Titsworth, S., \& Mazer, J. P. (2016). Teacher clarity: An analysis of current research and future directions. In P. L. Witt (Ed.), Handbooks of communication science: Communication and learning (pp. 105-128). De Gruyter Mouton. https://doi.org/10.1515/9781501502446-006

VanVoorhis, C. R. W., \& Morgan, B. L. (2007). Understanding power and rules of thumb for determining sample sizes. Tutorials in Quantitative Methods for Psychology, 3(2), 43-50. https://doi.org/10.20982/tqmp.03.2.p043

Wang, Z., \& Adesope, O. (2017). Do focused self-explanation prompts overcome seductive details? A multimedia study. Journal of Educational Technology \& Society, 20 (4), 4757. https://eric.ed.gov/?id=EJ1157893

Wang, F., Li, W., Mayer, R. E., \& Liu, H. (2018). Animated pedagogical agents as aids in multimedia learning: Effects on eye-fixations during learning and learning outcomes. Journal of Educational Psychology, 110(2), 250-268. http://doi.org/10.1037/edu0000221

Wang, Z., Sundararajan, N., Adesope, O. O., \& Ardasheva, Y. (2017). Moderating the seductive 
details effect in multimedia learning with note-taking. British Journal of Educational Technology, 48 (6), 1380-1389. http://doi.org/10.1111/bjet.12476

Wanzer, M. B., Frymier, A. B., \& Irwin, J. (2010). An explanation of the relationship between instructor humor and student learning: Instructional Humor Processing Theory. Communication Education, 59(1), 1-18. https://doi.org/10.1080/03634520903367238

Witt, P. L., Wheeless, L. P., \& Allen, M. (2004). A meta-analytical review of the relationship between teacher immediacy and student learning. Communication Monographs, 71(2), 184-207. https://doi.org/10.1080/036452042000228054

Wittrock, M. C. (1989). Generative processes of comprehension. Educational Psychologist, 24(4), 345-376. http://doi.org/10.1207/s15326985ep2404_2

Wang, F., Li, W., Mayer, R. E., \& Liu, H. (2018). Animated pedagogical agents as aids in multimedia learning: Effects on eye-fixations during learning and learning outcomes. Journal of Educational Psychology, 110(2), 250-268. http://doi.org/10.1037/edu0000221

Wang, N., Johnson, W. L., Mayer, R. E., Rizzo, P., Shaw, E., \& Collins, H. (2008). The politeness effect: Pedagogical agents and learning outcomes. International Journal of Human-Computer Studies, 66(2), 98-112. http://doi.org/10.1016/j.ijhcs.2007.09.003

Wong, A., Leahy, W., Marcus, N., \& Sweller, J. (2012). Cognitive load theory, the transient information effect and e-learning. Learning and Instruction, 22(6), 449-457. http://doi.org/10.1016/j.learninstruc.2012.05.004

Xie, H., Mayer, R. E., Wang, F., \& Zhou, Z. (2019). Coordinating visual and auditory cueing in multimedia learning. Journal of Educational Psychology, 111(2), 235-255. http://doi.org/10.1037/edu0000285 
Yeung, A. S., Jin, P., \& Sweller, J. (1998). Cognitive load and learner expertise: Split-attention and redundancy effects in reading with explanatory notes. Contemporary Educational Psychology, 23(1), 1-21. http://doi.org/10.1006/ceps.1997.0951

Yue, C. L., \& Bjork, E. L. (2017). Using selective redundancy to eliminate the seductive details effect. Applied Cognitive Psychology, 31(5), 565-571. http://doi.org/10.1002/acp.3348

Zheng, R. Z., \& Greenberg, K. (2018). The boundary of different approaches in cognitive load measurement: Strengths and limitations. In R. Z. Zheng (Ed.), Cognitive load measurement and application: A theoretical framework for meaningful research and practice (pp. 45-56). Routledge. https://doi.org/10.4324/9781315296258

Zimmerman, B. J. (2008). Investigating self-regulation and motivation: Historical background, methodological developments, and future prospects. American Educational Research Journal, 45(1), 166-183. https://doi.org/10.3102/0002831207312909

Zoom. (n.d.). Zoom for education. https://zoom.us/education 


\section{Appendix A}

\section{Pilot Study Lesson Script}

*The instructor shares their screen, displaying a PowerPoint presentation of an Introduction slide with the title "College Student Development." A live video image of the instructor is visible in the upper right corner of the screen.*

Hello, everyone. My name is [INSTRUCTOR NAME], and today's lesson is called "College Student Development."

In this lesson, we will be talking about what we know as far as how students like you develop a sense of identity throughout your college experiences, as well as identify some different factors that can affect how that happens. Let's get started!

*A new PowerPoint slide (slide 2) appears on the screen.*

In 1969, a researcher named Arthur Chickering developed a theory to describe how students discover who they are at college.

*The instructor draws a circle using the "draw" feature around the name "Arthur Chickering” on the screen as he says it.*

Even though identity and identity development are important topics for a person at any point during their lives, Chickering believed that establishing identity is a core developmental issue that people especially grapple with during their time in college. Chickering proposed that there are seven different vectors of development that each contribute to how college students' form their individual identities and sense of self - and he argued that these seven vectors encompass emotional, interpersonal, ethical, and intellectual aspects of our individual identities.

These vectors, specifically, are: (1) Developing Competence, (2) Managing Emotions, (3) Moving Through Autonomy Toward Interdependence, (4) Developing Mature Interpersonal Relationships, (5) Establishing Identity, (6) Developing Purpose, and (7) Developing Integrity.

*The instructor types the appropriate number next to each vector as he reads the name of the vector aloud (e.g., the instructor types " 1 " beside "Developing Competence" on the screen as he names the vector).*

Chickering described the seven vectors as "highways for journeying toward individuation." In other words, the seven vectors are different ways in which college students develop a sense of self and an understanding of who they are. The reason that Chickering labeled each of these as vectors is because identity development isn't necessarily a straight line or a step-by-step process. 
*The instructor underlines the word "vector." The instructor draws a red " $X$ " using the "draw" feature across the image of a straight error presented on the PowerPoint slide.*

In fact, we can progress or digress across each of these vectors with different intensity at different points in time - essentially moving up or down, left or right, forwards or backwards, or really in whatever direction our experiences end up taking us.

*The instructor draws a green checkmark using the "draw" feature in the white space included in the image of the multi-directional arrow.*

For example, just because you might have already established a sense of identity before you started college doesn't mean that your identity hasn't changed since your first semester. Instead, it's always more or less in a state of flux as you try new things, learn new information, and meet new people.

To make things even messier, Chickering suggested that college students can move through the seven vectors at different speeds, might have to deal with issues related to more than one vector at the same time, that vectors can interact with one another, and that college students frequently end up reexamining issues associated with vectors they may have previously resolved. Long story short, the ways that college students develop a sense of identity can get pretty complicated, and how well college students are able to navigate those complications can have a huge impact on how they ultimately come to see themselves as individuals.

Let's talk about each of Chickering's seven vectors of college student development in a little more detail to really flesh out what he was talking about.

*The instructor removes all markings from the screen. A new PowerPoint slide (slide 3) appears on the screen.*

The first vector that Chickering identified is the Developing Competence vector.

\section{*The instructor places a stamp of a star next to the words "Developing Competence" on the PowerPoint slide.*}

Competence is all about feeling confident - it's the belief that you have what it takes to overcome obstacles and achieve your goals. Chickering suggested that college students might feel more or less competent in different settings or based on what they are specifically trying to do at a particular point in time. In other words, Chickering didn't believe that someone is just competent all the time regardless of what they're doing, where they're at, or who they're with. Instead, he argued that there are three different types of competence that college students develop throughout their undergraduate experiences: intellectual competence, physical competence, and interpersonal competence. 
*The instructor uses the draw feature to draw a blue dot beside "intellectual competence," an orange dot beside "physical competence," and a purple dot beside “interpersonal competence."*

Chickering characterized a person's competence as a pitchfork with three tines, and he said that intellectual, physical, and interpersonal competence are each three metaphorical tines on the pitchfork. When college students develop intellectual, physical, and interpersonal competence - basically sharpening each tine of the pitchfork - their overall competence is enhanced.

*The instructor draws a blue line - the same color as the dot located beside "intellectual competence" - along the leftmost tine in the image of the pitchfork.*

Intellectual competence is the first tine on the pitchfork, and it basically involves learning new things. Intellectual competence is all about acquiring knowledge and skills related to a particular subject matter, or developing the ability to think critically and engage in complex reasoning. Intellectual competence, in a sense, is the reason you take a lot of the courses that you do during college - to develop knowledge about whatever the subject of a particular course is.

*The instructor draws an orange line - the same color as the dot located beside "physical competence" - along the middle tine in the image of the pitchfork.*

Physical competence, the second tine on Chickering's metaphorical pitchfork, refers to the development of manual skills or the ability to "do" something. Physical competencies closely related to athletic and recreational activities, attention to wellness, or involvement in artistic and manual activities. Playing a sport, exercising, painting, playing music - each of these is an example of physical competence because you have to develop some level of skill in order to do them.

*The instructor draws a purple line - the same color as the dot located beside "interpersonal competence"-along the rightmost tine in the image of the pitchfork.*

The last tine on the pitchfork is interpersonal competence, which is all about ability to communicate with others, be a leader, and working effectively in a team. If someone has developed interpersonal competence, it means that they can convey their thoughts and feelings to others effectively and appropriately. Do you know anyone who's able to make new friends easily? If you do, chances are that person is high in interpersonal competence - or, in other words, they have strong social skills.

So, before we move any further, let's make sure we understand what we have covered so far with a quick Zoom poll. 
I'm sure that most of you have had at least one experience during college where you needed to complete a task in a group. Think back on your group experience and answer this question for me - which of the three types of competence we just discussed is most closely related to how we work together with others in groups?

*The instructor activates a class poll. The prompt for the poll reads "Which of the three types of competence we just discussed is most closely related to how we work together with others in groups? "There are three responses to choose from: "Intellectual Competence," "Physical Competence," and "Interpersonal Competence." The instructor waits for ten seconds to allow students to select a response. After the ten seconds pass, the instructor closes the poll. The instructor reveals the correct answer, "Interpersonal Competence."*

Right! Although it's possible that you could be developing Intellectual and Physical Competence at the same time, learning to work together with others definitely aligns most closely with Interpersonal Competence.

*The instructor removes all markings from the screen. A new PowerPoint slide (slide 4) appears on the screen. The instructor places a stamp of a star next to the words "Managing Emotions" on the PowerPoint slide.*

Chickering's second vector of college student development is Managing Emotions. In the Managing Emotions vector, college students develop the ability to recognize and accept their own emotions, as well as the emotions of others. Just as important, it is in the Managing Emotions vector that we learn how to express our emotions appropriately.

You don't need me to tell you that college can be pretty chaotic. College students are dealing with constant changes personally, academically, and professionally all at once. These changes, in turn, can make us feel a lot of different ways.

Sometimes things happen that leave us feeling positive emotions like excitement, hope, or inspiration.

*The instructor types the "excitement, hope, inspiration" underneath the green smiling face in the image presented in the PowerPoint.*

Think of when you've done well on an exam when you weren't expecting to, or when you finally got a phone number from that person you've been interested in. When things are going well, it's good to take a moment to appreciate the positive emotions that we experience.

In contrast, sometimes things don't go our way and we experience more negative emotions like anxiety, depression, anger, or shame. 
*The instructor types the "anxiety, depression, anger, shame" underneath the red frowning face in the image presented in the PowerPoint.*

Plenty of things can go wrong in college. Maybe you've done something at a party that backfired and left you feeling embarrassed, or maybe you've been having problems in a relationship that is important to you as you navigate the challenges of a college environment. College is often described as just being a stressful time in general, and maybe you've been having a tough time handling that stress. Just like it's important to recognize when we're feeling positive, acknowledging our negative emotions is an important part of the Managing Emotions vector.

Different emotions can influence behavior in different ways, and we need to develop the ability to express our emotions constructively or restrain ourselves from engaging in emotional behavior that could negatively impact ourselves or the people around us. Essentially, the Managing Emotions vector is all about learning how to feel and how to act on those feelings.

You've probably already experienced examples of this firsthand.

*The instructor uses the draw feature to draw a red circle around the image of the red frowning face and the words "anxiety, depression, anger, shame."*

Can you think of a time in your own life when someone or something upset you, and you ended up doing something you regretted? Sometimes we become overwhelmed with negative emotions and lash out at the people around us yelling at them, insulting them, or maybe even blaming them for the negative emotions we're experiencing.

*The instructor uses the draw feature to draw a green circle around the image of the green smiling face and the words "excitement, hope, and inspiration."*

On the other hand, positive emotions can cloud our judgement, too. Sometimes we become so excited that we experience a sense of invulnerability, mistakenly believing that nothing can hurt us. Other times we get caught up in the momentum of the moment and get carried away - we're having such a good time that we forget to pause and really think about what we are doing. Because of this, learning how to navigate both our negative and positive emotions is critical as we progress through the Managing Emotions Vector.

*The instructor removes all markings from the screen. A new PowerPoint slide (slide 5) appears on the screen. The instructor places a stamp of a star next to the words " Moving Through Autonomy Toward Interdependence “on the PowerPoint slide.*

The third vector is Moving Through Autonomy Toward Interdependence. For many college students, the transition to a higher education environment is 
accompanied by a sense personal freedom that they have never experienced before.

*The instructor uses the draw feature to create a blue rectangle surrounding the single stick figure in the image presented on the PowerPoint slide.*

As students move away from home and begin living on their own, they become independent. They learn to stand on their own two feet and they're able to make important decisions for themselves rather than relying on instructions from a parental figure.

*The instructor uses the draw feature to overlay a brighter arrow over the arrow in the image presented on the PowerPoint slide while saying "they also develop new relationships with other college students enjoying their own independence."*

As students experience and enjoy their newfound autonomy, they also develop new relationships with other college students enjoying their own independence.

*The instructor uses the draw feature to create a yellow rectangle surrounding the group of stick figures in the image presented on the PowerPoint slide while saying "create new groups."*

As they grow closer, they create new groups - keeping their independence as individuals while simultaneously forming connections with others and learning to rely on one another. The Moving Through Autonomy Toward Interdependence vector is all about college coming to terms with the fact that they are both independent from and interdependent with others at the same time. In other words, we're self-sufficient individuals and members of larger groups simultaneously, and our actions affect other members of the groups that we belong to.

*The instructor removes all markings from the screen. A new PowerPoint slide (slide 6) appears on the screen. The instructor places a stamp of a star next to the words "Developing Mature Interpersonal Relationships" on the PowerPoint slide.*

Chickering's fourth vector is the Developing Mature Interpersonal Relationships vector. In this vector, Chickering suggested that college students develop tolerance and appreciation for intercultural and interpersonal differences, as well as the capacity for long-lasting and healthy relationships with others. In the Developing Mature Interpersonal Relationships vector, college students recognize that not everyone thinks, feels, looks, or lives the same way they do. They develop the ability to accept others for who they are, respect ways in which others are different, and appreciate areas of commonality wherever they may exist.

As an example, the Developing Mature Interpersonal Relationships vector played a huge part in my development as a college student. I grew up in a really small town where, for the most part, people were more similar than different. Everyone 
tended to dress the same, listen to the same music, have the same hobbies, have similar perspectives and beliefs, and there wasn't too much diversity. When I went to college, it was actually really jarring - in a good way - to meet people from many different walks of life. I met people with different religious beliefs, people from different cultural backgrounds, people with different sexual identities, and people with different political beliefs.

*The instructor uses the draw feature to create a yellow arrow pointing to the blue stick figure while saying "different religious beliefs," to the red stick figure while saying "different cultural backgrounds," to the purple stick figure while saying "different sexual identities," and to the green stick figure while saying "different political beliefs. "*

It provided me with awesome opportunities to think in ways that I never had before, exploring new ideas, new interests, and new ways of seeing the world. Sometimes we are instinctively tempted to close ourselves off from people who are different than us, but resisting that temptation is extremely important if we're going to progress through the Developing Mature Interpersonal Relationships vector.

*The instructor removes all markings from the screen. A new PowerPoint slide (slide 7) appears on the screen. The instructor places a stamp of a star next to the words "Establishing Identity" on the PowerPoint slide.*

The fifth vector is Establishing Identity. Chickering suggested that identity is extremely multifaceted - in other words, it's made up of a lot of different parts. Identity includes your comfort with your body and appearance, your gender and sexual orientation, your social or cultural heritage, your self-concept and selfesteem, and your sense of personal stability and integration. Given this, Chickering argued that our progress in the Establishing Identity vector is based on by the four vectors we we've already discussed in this lesson: Developing Competence, Managing Emotions, Moving Through Autonomy Toward Interdependence, and Developing Mature Interpersonal Relationships.

*While reading the name of each vector, the instructor uses the draw feature to draw arrows from each to a specific puzzle piece in the image presented on the PowerPoint slide.*

Each of these four vectors is basically a piece of a puzzle that all come together in the Establishing Identity vector, creating a fuller picture of who we are.

*The instructor removes all markings from the screen. A new PowerPoint slide (slide 8 ) appears on the screen. The instructor places a stamp of a star next to the words "Developing Purpose" on the PowerPoint slide.* 
The sixth vector is Developing Purpose. In the Developing Purpose vector, you basically figure out why you get up in the morning. This vector involves college students identifying and pursuing goals. Those goals can be vocational and careeroriented, or they might involve meaningful commitments to personal interests or things you enjoy. They might also be based on interpersonal commitments you've made or are in the process of making, like your obligations to new friends or a new significant other. The Developing Purpose vector is focused on asking yourself, "why?"

*The instructor uses the draw feature to write "WHY?" on the screen while saying " The Developing Purpose vector is focused on asking yourself, "why?"*

When Developing Purpose, college students attempt to find direction for themselves in life, asking themselves questions such as "Why am I here?" In a sense, the Developing Purpose vector is all about finding the reason that you're here and why you do what you do.

*The instructor removes all markings from the screen. A new PowerPoint slide (slide 9) appears on the screen.*

The seventh and final vector of Chickering's theory is the Developing Integrity vector. The Developing Integrity vector involves us recognizing that our moral and ethical values have an effect on our actions. Chickering suggested that college students progress through the Developing Integrity vector as they transition from making decisions based on rigid, moralistic thinking to systems of problemsolving and decision-making that strike a balance between self-interest and social responsibility. Basically, college students move through the Developing Integrity vector as they balance their personal value systems with the value systems held by the groups that they belong to.

*The instructor uses the draw feature to create an arrow from the text "Personal Value Systems" to one of the scales included in the rightmost image presented on the PowerPoint slide. The instructor uses the draw feature to create an arrow from the text "Group Value Systems" to the other scale included in the rightmost image presented on the PowerPoint slide.*

As we grow up, we are often told what is "right" or "wrong," and the world can come off to us as a bit black and white. We're often told that lying, for example, is always wrong and that we should always strive to be honest. As we move through the Developing Integrity vector, we start to re-examine ideas like this. What if the lie protects someone who we care about? What if the lie spares someone's feelings? What if the lie is intended to be helpful? Imagine someone asks you if you've been planning a surprise party for their birthday - do you spoil the surprise and ruin the birthday party for the sake of being honest? 
As college students, you've probably already found yourselves in plenty of situations that have challenged your pre-existing ideas of right and wrong. Another reason that college is such an important time for our identity development is that it forces us into situations where we begin to realize that the world is not as black and white as we might have originally thought.

*The instructor uses the draw feature to draw a red $X$ through the image of the sign displaying "Right $\rightarrow$, $\leftarrow$ Wrong”.*

Right and wrong are not always easy to distinguish from each other, and during college many students start to recognize and appreciate this.

So, that wraps up Chickering's seven vectors.

Before we go any further, let's double-check that we understand what we have covered so far with another quick Zoom poll.

One of the seven vectors we discussed, Establishing Identity, is particularly complicated because it's based on how we develop across four other vectors. Take a moment and think back as best you can about which vectors we said influence development in the Establishing Identity vector. Then, tell me which four vectors influence how we establish our identity on this Zoom poll.

*The instructor activates a class poll. The prompt for the poll reads "The Establishing Identity vectors is based on which of the following vectors? (Select all that apply)". There are six responses to choose from: (1) "Developing Competence", (2) "Managing Emotions", (3) "Moving Through Autonomy Toward Interdependence”, (4)

“Developing Mature Interpersonal Relationships", (5) “Developing Purpose”, and (6) “Developing Integrity”.*

Right! The ways that we develop in the Establishing Identity vector is based on the first four vectors we covered in this lesson: Developing Competence, Managing Emotions, Moving Through Autonomy Toward Interdependence, and Developing Mature Interpersonal Relationships. These four vectors are essentially puzzle pieces that fit together as we progress through the Establishing Identity vector. Don't worry if you had trouble with that one - like I said, it's a bit complicated.

*A new PowerPoint slide (slide 10) appears on the screen.*

Alright, moving on...

Chickering argued that how college students develop their identities across the seven vectors is heavily influence by their larger educational environment. In particular, he suggested that there are seven key environmental factors that can influence college 
students' identity development - factors he described as key influences. Let's discuss each of these seven key influences briefly:

*The instructor draws a circle using the draw feature to place a stamp of a star beside the words "Institutional Objectives."*

The first of the seven key influences that Chickering identified is Institutional Objectives. Institutional Objectives refer to the goals of a particular college or university. A lot of schools have a mission statement - an official articulation of the values or ideas that are important to them. Basically, think of this like a school's motto.

Not too surprisingly, this leads to different schools prioritizing different vectors of student development. For example, while some colleges or universities might prioritize developing students' competence - especially intellectual competence others might pay more attention to developing students' sense of integrity and ability to discern right from wrong. The vectors of identity development that a school identifies as most important often influence the ways in that school sets up its programs. As a result, it can have a huge impact on how students who attend a particular school develop their individual identities.

*The instructor removes all markings from the screen. The instructor places a stamp of a star beside the words "Institutional Size."*

The second key influence is Institutional Size. Chickering proposed that student participation in college life and satisfaction with the college experience go handin-hand, and college students' experiences may be diminished at larger colleges and universities. Students' progression across the seven vectors of identity development requires meaningful participation in opportunities provided by a college or university, which Chickering warned may not necessarily be provided at institutions with excessively high numbers of students. Larger schools typically offer programs, events, and other opportunities that cast a wide net - they are designed to be generally applicable to as many students as possible. For example, most people like pizza, so a large school might have a pizza night in the student commons. In contrast, Chickering suggested that smaller institutions are more likely to provide students with opportunities that are more custom-tailored to students' specific interests, needs, and goals. For example, maybe a small school that is primarily oriented towards the arts and humanities hosts an independent film contest or an art gallery for exhibiting students' work. With less students, smaller schools can offer pretty specific opportunities rather than opportunities that are one-size-fits-all.

*The instructor removes all markings from the screen. The instructor places a stamp of a star beside the words "Student-Faculty Relationships."* 
The third key influence is Student-Faculty Relationships. Chickering suggested that meaningful interaction between students and faculty plays a huge part in students' identity development. Because of this, he argued that students should be able to see their teachers in a variety of situations involving different roles and responsibilities rather than exclusively during class. As students begin to perceive faculty as real human beings who are genuinely interested in who they are and are accessible outside of the classroom, students and faculty receive opportunities to learn more about one another as individuals and create deeper personal, professional, and academic relationships that can enhance students' identity development. Basically, there's only so much that your teachers can learn about you during class. If you interact outside of class, you can create a meaningful relationship and, as they learn more about you, maybe they can provide you with unique opportunities you'd have never even heard about otherwise.

*The instructor removes all markings from the screen. The instructor places a stamp of a star beside the words "Curriculum."*

The fourth key influence is Curriculum. Chickering believed that the curriculum in the classes that each of you are taking has a tremendous influence on your identity development. Specifically, he argued that curriculum needs to be relevant to your interests, your needs, your goals, and your experiences, as well as offer diverse perspectives that represent a variety of values and beliefs. Have you ever taken a class just because you needed a certain type of course credit - like a general elective - and found yourself wondering "What the heck does this topic have to do with my life?" According to Chickering, that should never happen the curriculum in your classes should be curriculum that you can actually use, not information that you forget and never need to apply again after a semester ends.

*The instructor removes all markings from the screen. The instructor places a stamp of a star beside the words "Teaching."*

The fifth key influence is Teaching. Chickering suggested that students' learning - and ultimately identity development - occurs when teachers at colleges and universities incorporate active learning, ongoing interaction with students inside and outside of class, timely and specific feedback, high expectations, and respect for individual learning differences into their instruction. In these ways, Chickering proposed that teachers can stimulate college students' progression across multiple vectors of identity development at the same time rather than focusing exclusively on students' intellectual competence. In other words, when your students understand the ways in which you learn, teach in those ways, and engage you in the learning process, your identity development will be enhanced.

*The instructor removes all markings from the screen. The instructor places a stamp of a star beside the words "Friendships and Student Committees." 
The sixth key influence is Friendships and Student Communities. Chickering once wrote that "a student's most important teacher is often another student." Basically, Chickering proposed that college students' development across all seven vectors is enhanced when students form meaningful friendships and participate in diverse student communities characterized by shared interests and ongoing interactions. Communities that college students participate in can be informal groups, like a circle of friends, or formal groups like residence hall floors, sports teams, student organizations, or classes. Communities that stimulate college students' development typically encourage regular communication and interaction between students, offer students opportunities to work together, are small and intimate enough so that no one feels redundant or left out, and are comprised of individuals from diverse backgrounds.

*The instructor removes all markings from the screen. The instructor places a stamp of a star beside the words "Student Development Programs and Services."*

The seventh and final key influence is Student Development Programs and Services. Chickering argued that it is important for faculty and student affairs professionals at colleges and universities to provide programs and services specifically designed to assist students in exploring and developing their individual identities. Rather than focusing solely on students' performance in the classroom, Chickering proposed that "educators" should instead refer to themselves as "student development professionals" and serve as advocates for the development of the whole student. By providing formal programs strategically designed to assist students' self-exploration and development, faculty and staff can provide valuable assistance to college students' progression across all seven vectors of identity development.

*The instructor removes all markings from the screen.*

Alright - so we moved through those seven key influences pretty fast compared to the seven vectors. Before we wrap up the lesson, let's make we are all on the same page with one last Zoom poll.

Each of you are already taking classes here at WVU, and those classes are taught by different instructors. According to Chickering, your identity development is enhanced when you are able to interact with those instructors inside and outside of the classroom because it allows you to learn more about each other as people. This idea is the main point behind which of the following key influences?

*The instructor activates a class poll. The prompt for the poll reads "Which of the seven key influences involves students and instructors interacting both inside and outside of the classroom?" There are seven responses to choose from: "Institutional Objectives," "Institutional Size," "Student-Faculty Relationships," "Curriculum," “Teaching," "Friendships and Student Committees," and "Student Development 
Programs and Services." The instructor waits for ten seconds to allow students to select a response. After the ten seconds pass, the instructor closes the poll. The instructor reveals the correct answer, "Student-Faculty Relationships."*

Right! Chickering argued that Student-Faculty Relationships play a huge part in how we develop a sense of identity during college, and he said that these relationships are more meaningful when we don't just see our instructors during class time. Thank you!

*A new PowerPoint slide (slide 11) appears on the screen.*

And that will do it for the seven key influences.

In this lesson, we discussed Arthur Chickering's theory of college student development, as well as identified different institutional factors which can influence the ways in which college students' identity development takes place. In particular, we reviewed Chickering's seven vectors of development and seven environmental factors - or key influences - that he argued can influence the extent to which college students' development occurs.

Thank you for participating in this lesson! In the chat, you will find a hyperlink to a brief survey that will ask you questions related to the lesson that you just participated in. Have a great day!

*The instructor ends the lesson.* 


\section{Appendix B}

Pilot Study PowerPoint

\section{College Student Development}

Theory of College Student Development

- Arthur Chickering's Seven Vectors

- Developing Competence

- Managing Emotions

- Moving Through Autonomy Toward Interdependence

- Developing Mature Interpersonal Relationships

- Establishing Identity

- Developing Purpose

- Developing Integrity

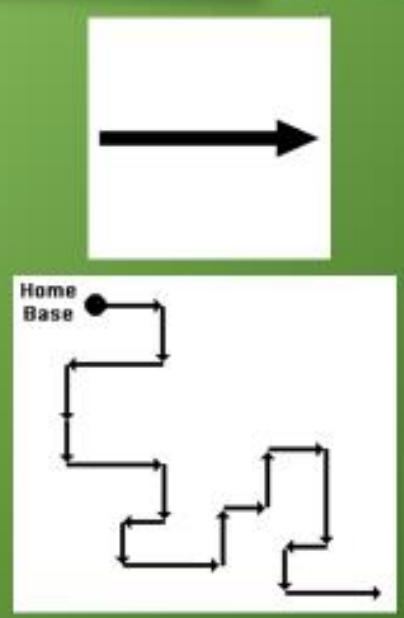




\section{The Vectors}

- Developing Competence

- Intellectual competence

- Physical competence

- Interpersonal Competence

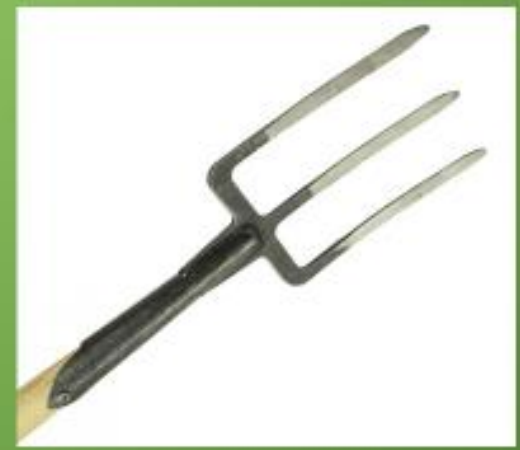

\section{The Vectors}

- Managing Emotions

- Negative Emotions

- Positive Emotions

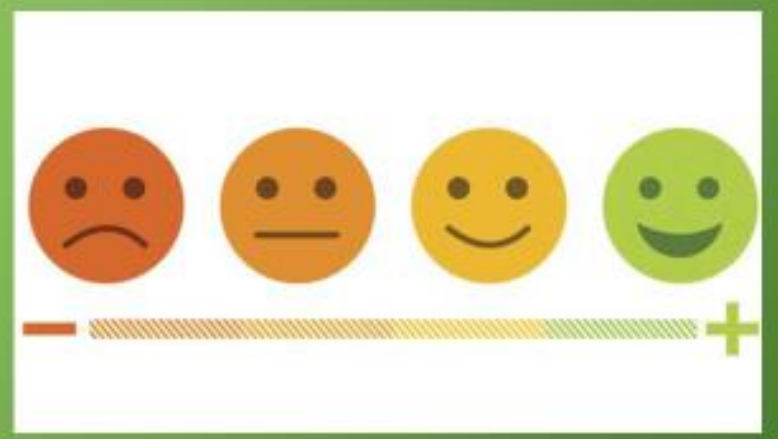




\section{The Vectors}

- Moving Through Autonomy Toward

Interdependence

- Discovering Autonomy

- Forming Connections

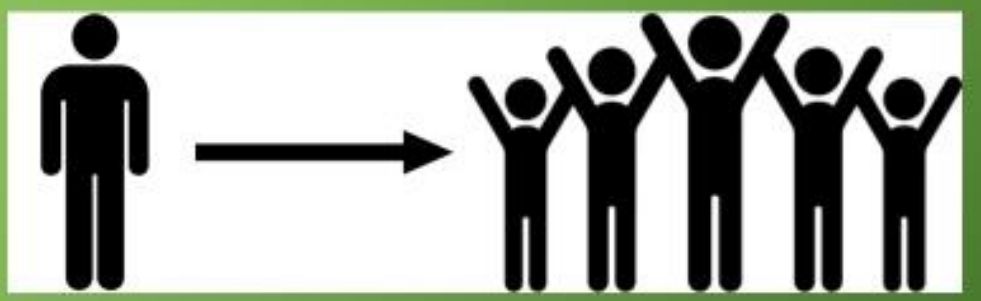

\section{The Vectors}

- Developing Mature Interpersonal Relationships

- Intercultural Differences

- Interpersonal Differences

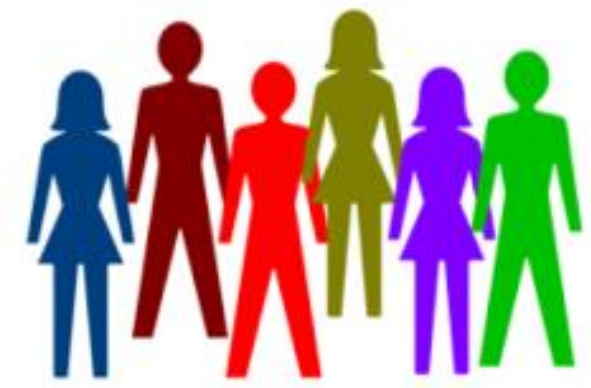




\section{The Vectors}

- Establishing Identity

- Developing Competence

- Managing Emotions

- Moving Through Autonomy Toward Interdependence

- Developing Mature Interpersonal Relationships

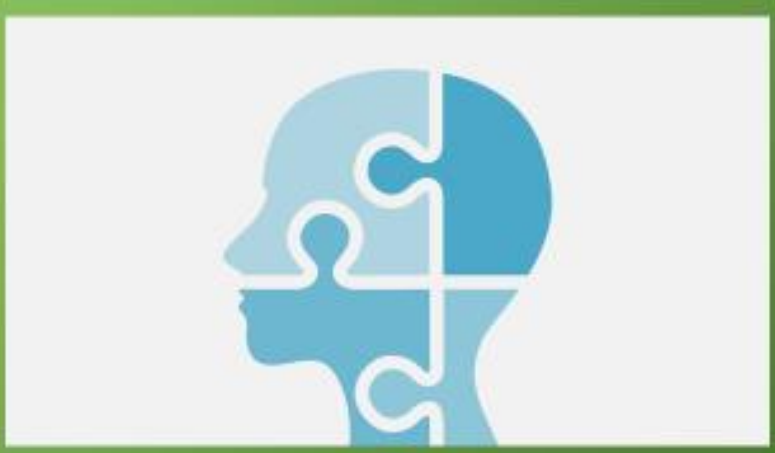

\section{The Vectors}

- Developing Purpose

- Vocational Goals

- Personal Goals

- Interpersonal Goals 


\section{The Vectors}

- Developing Integrity

- Personal Value Systems

- Group Value Systems

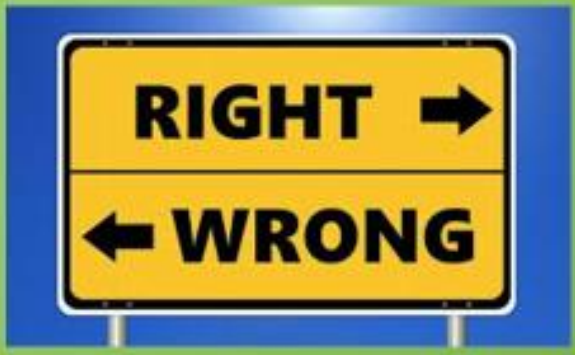

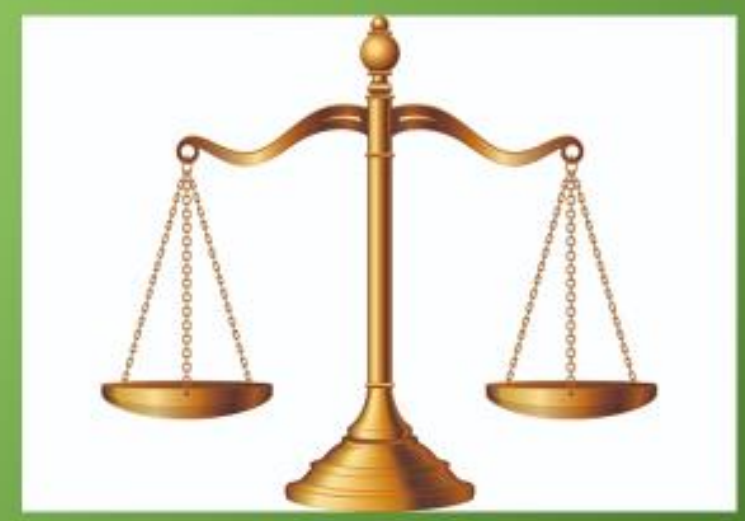

\section{Key Influences}

- Institutional Objectives

- Institutional Size

- Student-Faculty Relationships

- Curriculum

- Teaching

- Friendships and Student Committees

- Student Development Programs and Services 


\section{Recap}

- Seven Vectors

- Seven Key Influences 


\section{Appendix C}

\section{Pilot Test Survey}

Dear Participant:

You are being asked to participate in a research study conducted by Principal Investigator Dr. Alan K. Goodboy and Co-Investigator Kevin C. Knoster, both of whom are in the Department of Communication Studies at West Virginia University. You must be 18 years of age or older to participate in this study. You are being asked to participate in an online lesson on "College Student Development" and complete a survey following the lesson. Completing and submitting the post-lesson survey indicates that you have agreed to participate in this study. Should you choose to take part in this study, please participate in the online lesson and complete the postlesson survey independently using either a computer or tablet. While in the lesson, please maximize the video of the lesson to fill your screen.

Your responses on the post-lesson survey will be kept completely anonymous. This study and its accompanying survey is in no way linked to either you or your course instructor. Do not put enter your name at any time while participating in this study in order to ensure anonymity. Participation in this study is completely voluntary. You may stop participating in the online lesson, skip certain questions if you want, and may stop completing the survey at any time without fear of penalty. There are no right or wrong answers. Your class standing, your class grades, or status in any athletic or other activity associated with West Virginia University cannot be affected by either your refusal to participate in, or withdrawal from participation in, this study. There are no known risks associated with participation in this study. The findings from this research will be used to develop future studies. Your participation in this study will take approximately 30 minutes.

If you would like more information about this research project, feel free to contact CoInvestigator Kevin C. Knoster at kcknoster@mix.wvu.edu. This study has been reviewed and acknowledged by West Virginia University's Institutional Review Board, and is on file as Protocol \#2101214973.

Thank you for your participation.

Sincerely,

Dr. Alan K. Goodboy

Professor

Principal Investigator

agoodboy@mail.wvu.edu
Kevin C. Knoster

Ph.D. Candidate

Co-Investigator

kcknoster@mix.wvu.edu 
You will now be presented with a series of questions regarding your perceptions of the lesson you just participated in. Please follow the instructions that follow as you respond to each question.

Please indicate the extent to which you agree or disagree with the following statements in regard to the lesson you just participated in.

\begin{tabular}{|c|c|c|c|c|c|c|}
\hline $\begin{array}{c}\text { Strongly } \\
\text { Disagree }\end{array}$ & $\begin{array}{c}\text { Moderately } \\
\text { Disagree }\end{array}$ & $\begin{array}{c}\text { Slightly } \\
\text { Disagree }\end{array}$ & $\begin{array}{c}\text { Neither } \\
\text { Disagree } \\
\text { nor Agree }\end{array}$ & $\begin{array}{c}\text { Slightly } \\
\text { Agree }\end{array}$ & $\begin{array}{c}\text { Moderately } \\
\text { Agree }\end{array}$ & $\begin{array}{c}\text { Strongly } \\
\text { Agree }\end{array}$ \\
1 & 2 & 3 & 4 & 5 & 6 & 7 \\
\hline
\end{tabular}

1. I found it easy to identify the crucial aspects of this lesson.

2. I was able to recognize what information was most relevant to learning the material.

3. I had the ability to differentiate what was important to know in this lesson from what was not.

4. I could determine what was significant to pay attention to in this lesson.

5. I knew what aspects of this lesson to concentrate on.

6. I understood what I was supposed to focus on during this lesson.

7. Was the instructor visible during the lesson you just participated in?

Yes or No

Please indicate the extent to which you believe the instructor of the lesson you just participated in engaged in each of the following behaviors.

\begin{tabular}{|c|c|c|c|c|}
\hline Never & Rarely & Occasionally & Often & Very Often \\
$\mathbf{1}$ & $\mathbf{2}$ & $\mathbf{3}$ & $\mathbf{4}$ & $\mathbf{5}$ \\
\hline
\end{tabular}

8. He used his hands and arms to gesture while teaching the lesson.

9. He used a monotone or dull voice while teaching the lesson.

10. He looked away from the camera while teaching the lesson.

11. He had a relaxed body position while teaching the lesson.

12. He frowned while teaching the lesson.

13. He avoided looking into the camera while teaching the lesson.

14. He had a tense body position while teaching the lesson.

15. His voice was monotonous or dull while teaching the lesson.

16. He used a variety of vocal expressions while teaching the lesson.

17. He gestured while teaching the lesson.

18. He was animated while teaching the lesson.

19. He had a bland facial expression while teaching the lesson.

20. He looked directly into the camera while teaching the lesson. 
21. He was stiff while teaching the lesson.

22. He had a lot of vocal variety while teaching the lesson.

23. He avoided gesturing while teaching the lesson.

24. He maintained eye contact through the camera while teaching the lesson.

25. He smiled while teaching the lesson.

Please indicate the extent to which you agree or disagree with the following statements regarding the Zoom polls included in the lesson you just participated in.

\begin{tabular}{|c|c|c|c|c|c|c|}
\hline $\begin{array}{c}\text { Strongly } \\
\text { Disagree }\end{array}$ & $\begin{array}{c}\text { Moderately } \\
\text { Disagree }\end{array}$ & $\begin{array}{c}\text { Slightly } \\
\text { Disagree }\end{array}$ & $\begin{array}{c}\text { Neither } \\
\text { Disagree } \\
\text { nor Agree }\end{array}$ & $\begin{array}{c}\text { Slightly } \\
\text { Agree }\end{array}$ & $\begin{array}{c}\text { Moderately } \\
\text { Agree }\end{array}$ & $\begin{array}{c}\text { Strongly } \\
\text { Agree }\end{array}$ \\
1 & 2 & 3 & 4 & 5 & 6 & 7 \\
\hline
\end{tabular}

26. The three polls helped me organize the material presented in this lesson in a logical manner.

27. The three polls helped me connect the ideas in this lesson to one another in a coherent fashion.

28. The three polls helped me understand the relationships between the various parts of this lesson.

29. The three polls helped me logically model concepts from this lesson as they applied to one another.

30. The three polls helped me grasp how the various parts of this lesson worked jointly to form the ideas I was learning.

Please indicate the extent to which you agree or disagree with the following statements in regard to the lesson you just participated in.

\begin{tabular}{|c|c|c|c|c|c|c|}
\hline $\begin{array}{c}\text { Strongly } \\
\text { Disagree }\end{array}$ & $\begin{array}{c}\text { Moderately } \\
\text { Disagree }\end{array}$ & $\begin{array}{c}\text { Slightly } \\
\text { Disagree }\end{array}$ & $\begin{array}{c}\text { Neither } \\
\text { Disagree } \\
\text { nor Agree }\end{array}$ & $\begin{array}{c}\text { Slightly } \\
\text { Agree }\end{array}$ & $\begin{array}{c}\text { Moderately } \\
\text { Agree }\end{array}$ & $\begin{array}{c}\text { Strongly } \\
\text { Agree }\end{array}$ \\
1 & 2 & 3 & 4 & 5 & 6 & 7 \\
\hline
\end{tabular}

31. The instructor taught this online lesson in a way that could possibly happen in real life.

32. The online lesson taught by the instructor portrayed a possible real-life online lesson.

33. The online lesson could actually happen in real life.

34. Never in a real online classroom would someone teach the way that this instructor taught this online lesson.

35. A real instructor would never teach an online lesson the way that this instructor did. 
You will now be asked to respond to 10 questions which will assess your knowledge of college student development based upon the lesson you just participated in. Please select the best answer for each question. You will not be able to return to previous questions once you have provided a response, so please consider your answers carefully.

1. The Theory of College Student Development was created by
a) Stanley Milgram
b) Arthur Chickering
c) Albert Bandura
d) Lawrence Kohlberg

2. According to the Theory of College Student Development, there are vectors that students move through while developing their identity.
a) Three
b) Five
c) Seven
d) Nine

3. According to the Theory of College Student Development, there are key influences that can affect college students' identity development.
a) Three
b) Five
c) Seven
d) Nine

4. The vector of college student development has been described as a three-tined pitchfork because it has three different dimensions.
a) Developing Competence
b) Managing Emotions
c) Establishing Identity
d) Developing Integrity

5. The vector of college student development is based on college students' development in the Developing Competence vector, Managing Emotions vector, Moving Through Autonomy Toward Interdependence vector, and Developing Mature Relationships vector.
a) Establishing Identity
b) Developing Integrity
c) Developing Purpose
d) Student-Faculty Relationships

6. While moving through the vector, college students develop tolerance and appreciation for intercultural differences.

a) Moving Through Autonomy Toward Interdependence 

b) Developing Mature Interpersonal Relationships
c) Developing Purpose
d) Developing Integrity

7. College students ask themselves questions such as "Why do I get up in the morning?" and "Why am I here?" while moving through the vector.
a) Establishing Identity
b) Developing Mature Interpersonal Relationships
c) Developing Purpose
d) Developing Integrity

8. College students' identity development is enhanced when they have interactions with their instructors outside of class, thus are/is important at colleges and universities.
a) Teaching
b) Student Development Programs and Services
c) Friendships and Student Committees
d) Student-Faculty Relationships

9. Different colleges and universities prioritize different aspects of college students' identity development, meaning that can significantly influence how students develop their identities at a particular school.
a) Institutional Objectives
b) Institutional Size
c) Student Development Programs and Services
d) Friendships and Student Committees

10. College students try to find a balance between their personal value systems and the value systems of the groups they belong to as they move through the vector of college student development.
a) Developing Competence
b) Developing Integrity
c) Establishing Identity
d) Managing Emotions

Finally, please provide us with some general information about yourself.

1. What is your age?

2. What sex do you identify as?

3. What is your class rank? (e.g., first-year, sophomore, junior, senior)

4. What ethnicity do you most closely identify with? 


\section{Appendix D}

Bulletin Board and Online Study Announcement

Title: Online Teaching Study

Protocol Number: 2102231619

PI: Dr. Alan K. Goodboy

PI E-mail: agoodboy@mail.wvu.edu

Co-PI: Kevin C. Knoster

Co-PI E-mail: kcknoster@mix.wvu.edu

Purpose of Study (1 sentence): The purpose of this research study is to examine the effects of different instructor communication behaviors on learning in a live online lesson.

To be eligible for participation in this study, you must meet the following inclusion criteria: You are only eligible to participate in this research study if: (1) you are a student at West Virginia University, (2) you are currently enrolled in a Communication Studies course, (3) you are over the age of 18, and (4) you are available to attend a live online lesson on the following dates/times: [insert dates and times]

Time Commitment: 60 minutes (Part $1=10$ minutes; Part $2=50$ minutes)

Data Collection Location: Online

Data Collection Date \& Time:

Part 1 Online Sign-Up Survey Link:

https://wvu.qualtrics.com/jfe/form/SV_3CPnvUGTNFVgo3Y

Part 2 Live Online Lesson Dates and Times:

[insert dates and times] 


\section{Appendix E}

Cover Letter

Dear Participant:

You are invited to take part in a research study entitled "Online Teaching Study," which is designed to examine college students' perceptions of instructor communication behaviors during a live online lesson. The purpose of this research study is to understand the effects of different instructional strategies on student learning in a live online lesson. This project is being conducted by Principal Investigator Dr. Alan K. Goodboy and Co-Investigator Kevin C. Knoster, both of whom are in the Department of Communication Studies at West Virginia University.

To participate in this 2-Part study, you must be: (1) a student at West Virginia University, (2) currently enrolled in a Communication Studies course, (3) at least 18 years old, and (4) available to attend a live online lesson on the following dates/times: [INSERT DATES AND TIMES].

If you are interested in participating in this research study, please complete this Part 1 online sign-up survey. The sign-up survey will take approximately 10 minutes to complete and will randomly assign you to a live online lesson at one of the dates and times indicated above. After being randomly assigned to your live online lesson, you will be provided with a hyperlink and password to access your assigned lesson and be prompted to create a unique alphanumeric identification code to register for your assigned lesson.

To complete Part 2 of this research study, you must attend the online lesson which you are randomly assigned to. Upon accessing your assigned online lesson on its specified date and time using the hyperlink and password provided in this Part 1 sign-up survey, you will participate in an online lesson and complete a subsequent post-lesson survey. The link to the post-lesson survey will be provided to you at the end of the online lesson. Part 2 of this research study should take approximately 50 minutes to complete.

Your participation in this research study will remain completely anonymous. Although the online lesson included in Part 2 will be recorded, you will use your unique alphanumeric identification code instead of your name, and you will neither appear on camera nor be asked to speak. There are no right or wrong answers to any questions on any survey, and your participation is completely voluntary. You may skip certain questions or stop completing surveys at any time without fear of penalty. Your actual performance in this research study will in no way impact your class standing, grades, job status, or status in any athletic or other activity associated with West Virginia University. There are no known risks associated with participating in this study.

As a student enrolled in a Communication Studies course, you may be eligible to receive research credit (extra credit) for participation in this study. To find out if you are eligible, please contact your Communication Studies course instructor and/or review your course syllabus. Your course syllabus should also include details regarding how much extra credit you may be eligible for (as well as how many research opportunities you can attempt for that class). Students not wishing to volunteer for this study are able to receive extra credit by completing an alternative assignment. For students in eligible classes, your instructor will provide more information on the 
alternative assignment. If you are seeking extra credit for your participation in this study, you will be provided with a hyperlink to a research receipt survey (worth a total of 60 minutes of research extra credit) upon completing the post-lesson survey at the end of the Part 2 live online lesson. In order to receive extra credit, you must attend the Part 2 live online lesson which you are randomly assigned to and provide the unique alphanumeric identification code you use to register for that lesson.

If you would like more information about this research project, feel free to contact coinvestigator Kevin C. Knoster at kcknoster@mix.wvu.edu. This study has been reviewed and acknowledged by West Virginia University's Institutional Review Board, and is on file as Protocol \#2102231619.

Thank you for your participation.

Sincerely,

Dr. Alan K. Goodboy

Professor

Principal Investigator agoodboy@mail.wvu.edu
Kevin C. Knoster

Ph.D. Candidate

Co-Investigator

kcknoster@mix.wvu.edu 
Appendix F

IRB-Approved Email Announcement

If you are a student at West Virginia University, currently enrolled in a Communication Studies course, and are at least 18 years old, you are eligible to participate in a WVU IRB approved research study examining how students perceive instructor communication behaviors in an online classroom. This is a 2-PART research study that adds up to a total of 60 minutes of research extra credit.

\section{Part 1:}

Part 1 is an online Qualtrics survey through which you will sign up to participate in Part 2 of this research study. The Part 1 sign-up survey will take approximately 10 minutes to complete and will provide you with the information you need to complete Part 2. You must complete the Part 1 online sign-up survey in order to be eligible to participate in Part 2 of the study.

\section{Part 2:}

Part 2 is a live online lesson which will take place on one of the following dates/times: [INSERT DATES AND TIMES]

You will be randomly assigned to attend one of these online lessons, thus you must be available to potentially attend both. Upon completing the lesson, you will be presented with a subsequent post-lesson surveys that will ask you a series of questions related to the lesson you attended. The Part 2 live online lesson and post-lesson survey will take approximately 50 minutes to complete. You will receive a link to an online research receipt survey (worth a total of 60 minutes of research extra credit) at the end of the Part 2 post-lesson survey. You must complete both Part 1 and part 2 to receive extra credit for your participation in this study.

Eligible students may earn extra credit for participation in this research study. To find out if you are eligible, please contact your instructor and/or consult your course syllabus policy on extra credit. There are $\mathbf{2}$ Parts to this study that add up to 60 minutes of research extra credit (Part $\mathbf{1}=$ 10 minutes; Part 2 = 50 minutes).

This research study is being conducted by Principal Investigator Dr. Alan K. Goodboy and CoInvestigator Kevin C. Knoster in the Department of Communication Studies at West Virginia University. If you would like more information about this research project, feel free to contact co-investigator Kevin C. Knoster at kcknoster@mix.wvu.edu. This study has been reviewed and acknowledged by West Virginia University's Institutional Review Board, and is on file as Protocol \#2102231619.

If you are eligible for this study and are available for all of the listed dates/times, please follow the link below to the Part 1 online sign-up survey.

Part 1 Online Sign-Up Survey: https://wvu.qualtrics.com/jfe/form/SV_3CPnvUGTNFVgo3Y

Thank you for your time and have a great day, 
Dr. Alan K. Goodboy

Professor

Principal Investigator

agoodboy@mail.wvu.edu
Kevin C. Knoster

$\mathrm{Ph} . \mathrm{D}$. Candidate

Co-Investigator

kcknoster@mix.wvu.edu 


\author{
Appendix G \\ Online Sign-Up Survey
}

Thank you for agreeing to participate in this research study. As outlined in the cover letter, you will be randomly assigned a date/time to attend the Part 2 live online lesson. Upon being assigned to this lesson, you will be asked to create a unique alphanumeric identification code which you will use to register for your assigned lesson. Please click the "next" button below to receive a date/time to attend the Part 2 live online lesson.

\title{
(If CTML-based lesson assigned)
}

You have been assigned to attend the following live online lesson:

Date: [INSERT DATE]

Time: [INSERT TIME].

You may access this live online lesson using the following link: [INSERT HYPERLINK]

Password: [INSERT PASSWORD]

Please take a moment to record this date, time, hyperlink, and password - you will not be provided with them again once you continue. In order to complete Part 2 of this study and receive research extra credit, you must access the lesson BEFORE it begins at [INSERT START TIME]. At [INSERT START TIME] the lesson will be locked as it begins and you will no longer be able to access it using the hyperlink provided. You must attend this online lesson which you were randomly assigned to. If you attempt to attend a different live online lesson which you were not assigned to, you will not be eligible to receive research extra credit for your participation in this study.

To finalize your registration for this research study, we need you to create a unique identification code. To be clear, this unique identification code will only be used to ensure that participants (1) remain anonymous during the Part 2 online lesson and survey and (2) attend the live online lesson to which they are assigned. This identification code will be removed before data analysis. Remember, your actual performance in this research study will in no way impact your class standing, grades, job status, or status in any athletic or other activity associated with West Virginia University

Please create your unique identification code using the following directions. Please provide the first three letters of the name of the town in which you were born and the last four digits of your telephone number (for example: NEW2651, MOR5473). Please keep this code for your records, as you will be asked to use it as your name during the live online lesson, as well as provide it on the research receipt survey if you are seeking extra credit for participating in this study.

My unique identification code is:

Please click "next" to complete your registration... 
You have just completed Part 1 of this research study. Thank you for creating your unique identification code and registering for your assigned Part 2 live online lesson!

Please remember to use the unique identification code you created instead of your name when connecting to your assigned online lesson. When you connect to the lesson, please do so using either a computer or tablet and maximize the video of the lesson to fill your screen. Please also remember to access your assigned lesson using the hyperlink and password provided BEFORE the lesson begins on its specified date and time. Once the lesson begins, access will be blocked and you will no longer be able to connect or participate.

We look forward to seeing you at your assigned lesson!

\section{(If non-CTML-based lesson assigned)}

You have been assigned to attend the following live online lesson:

Date: [INSERT DATE]

Time: [INSERT TIME].

You may access this live online lesson using the following link: [INSERT HYPERLINK]

Password: [INSERT PASSWORD]

Please take a moment to record this date, time, hyperlink, and password - you will not be provided with them again once you continue. In order to complete Part 2 of this study and receive research extra credit, you must access the lesson BEFORE it begins at [INSERT START TIME]. At [INSERT START TIME] the lesson will be locked as it begins and you will no longer be able to access it using the hyperlink provided. You must attend this online lesson which you were randomly assigned to. If you attempt to attend a different live online lesson which you were not assigned to, you will not be eligible to receive research extra credit for your participation in this study.

To finalize your registration for this research study, we need you to create a unique identification code. To be clear, this unique identification code will only be used to ensure that participants (1) remain anonymous during the Part 2 online lesson and survey and (2) attend the live online lesson to which they are assigned. This identification code will be removed before data analysis. Remember, your actual performance in this research study will in no way impact your class standing, grades, job status, or status in any athletic or other activity associated with West Virginia University

Please create your unique identification code using the following directions. Please provide the first three letters of the name of the town in which you were born and the last four digits of your telephone number (for example: NEW2651, MOR5473). Please keep this code for your records, as you will be asked to use it as your name during the live online lesson, as well as provide it on the research receipt survey if you are seeking extra credit for participating in this study. 
My unique identification code is:

Please click "next" to complete your registration...

You have just completed Part 1 of this research study. Thank you for creating your unique identification code and registering for your assigned Part 2 live online lesson!

Please remember to use the unique identification code you created instead of your name when connecting to your assigned online lesson. When you connect to the lesson, please do so using either a computer or tablet and maximize the video of the lesson to fill your screen. Please also remember to access your assigned lesson using the hyperlink and password provided BEFORE the lesson begins on its specified date and time. Once the lesson begins, access will be blocked and you will no longer be able to connect or participate.

We look forward to seeing you at your assigned lesson! 


\section{Appendix $\mathrm{H}$}

PowerPoint Presentation (Main Experiment)

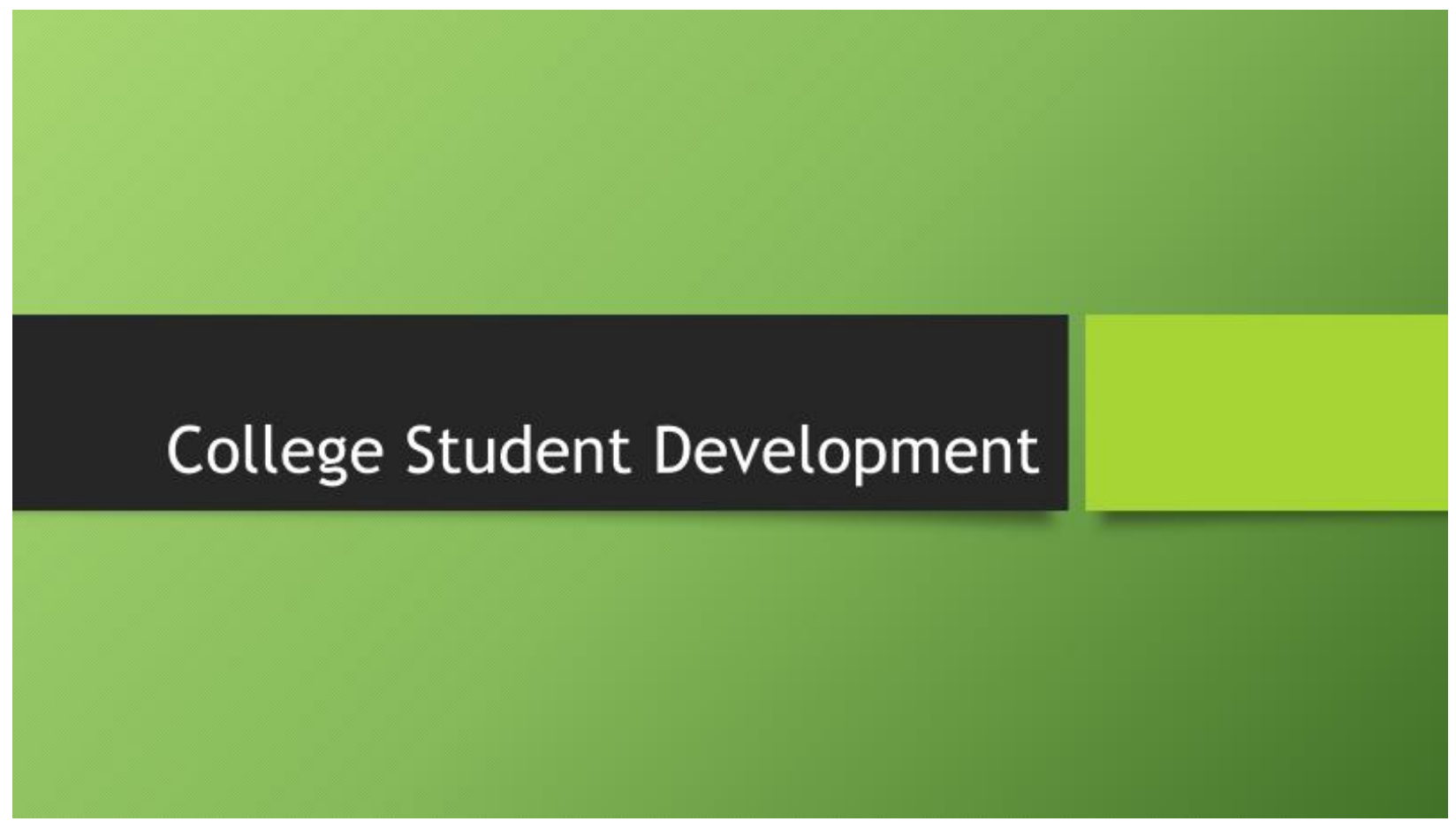

\section{Theory of College Student Development}

- Arthur Chickering's Seven Vectors

- Developing Competence

- Managing Emotions

- Moving Through Autonomy Toward Interdependence

- Developing Mature Interpersonal Relationships

- Establishing Identity

- Developing Purpose

- Developing Integrity

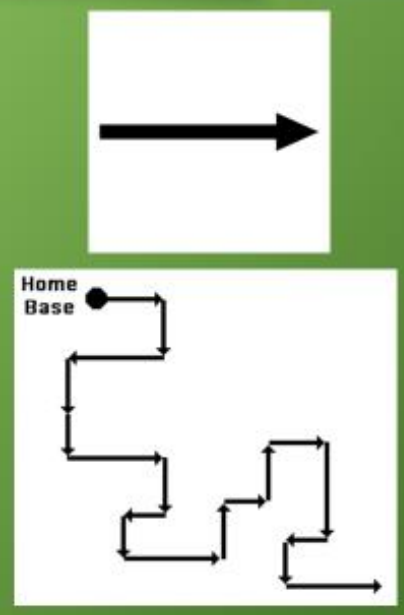




\section{The Vectors}

- Developing Competence

- Intellectual Competence

- Physical Competence

- Interpersonal Competence

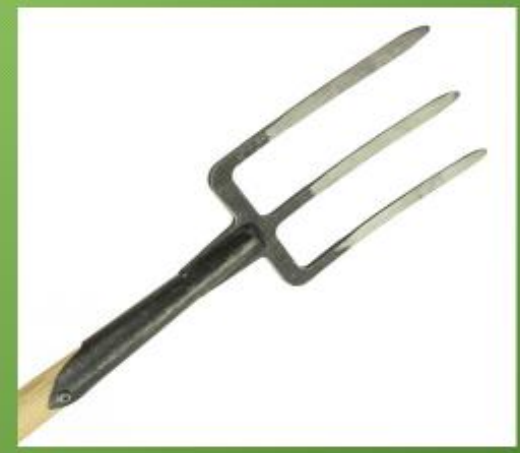

\section{The Vectors}

- Managing Emotions

- Negative Emotions

- Positive Emotions

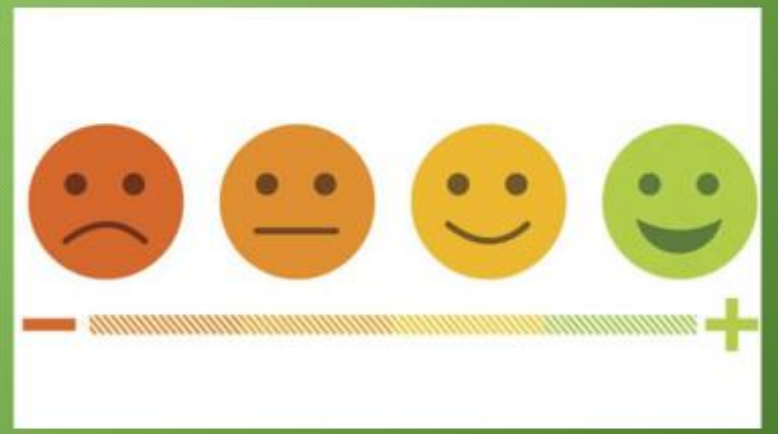




\section{The Vectors}

- Moving Through Autonomy Toward Interdependence

- Discovering Autonomy

- Forming Connections

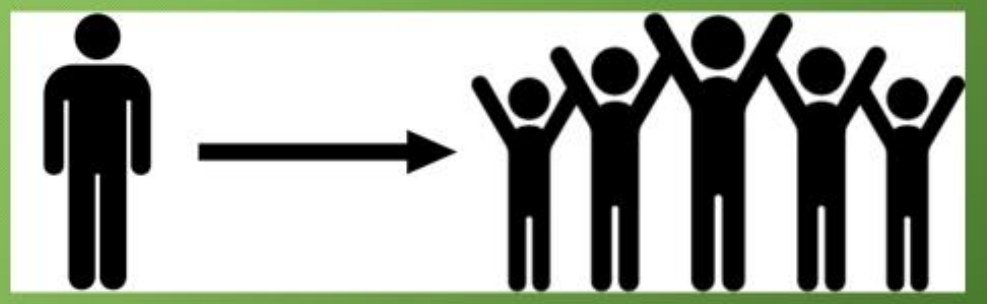

\section{The Vectors}

- Developing Mature Interpersonal Relationships

- Intercultural Differences

- Interpersonal Differences

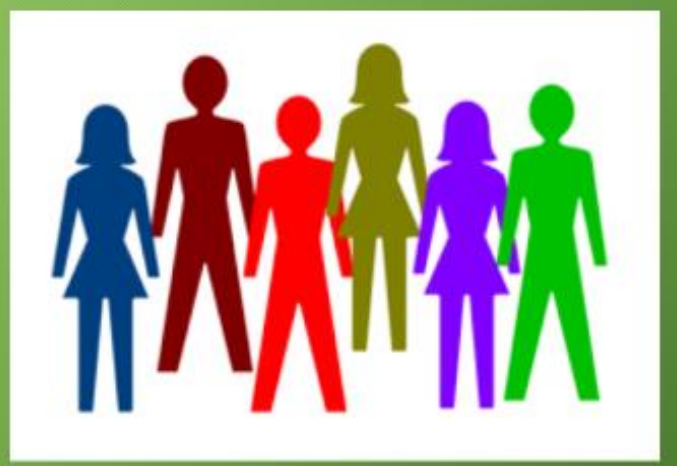




\section{The Vectors}

- Establishing Identity

- Developing Competence

- Managing Emotions

- Moving Through Autonomy Toward Interdependence

- Developing Mature Interpersonal Relationships

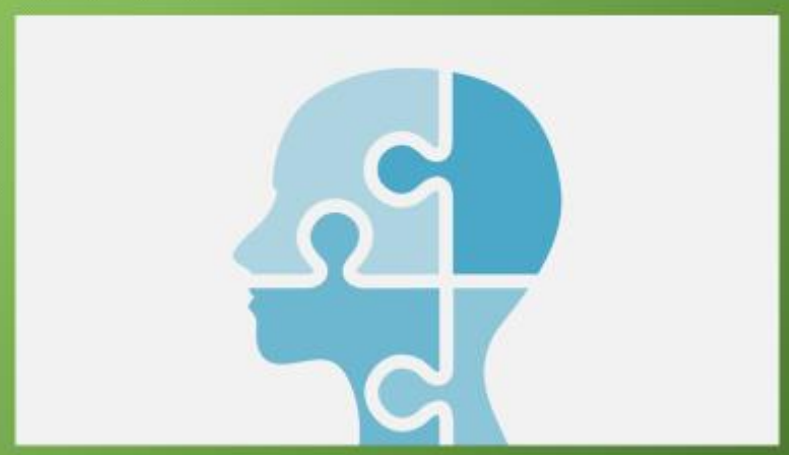

\section{The Vectors}

- Developing Purpose

- Vocational Goals

- Personal Goals

- Interpersonal Goals 


\section{The Vectors}

- Developing Integrity

- Personal Value Systems

- Group Value Systems
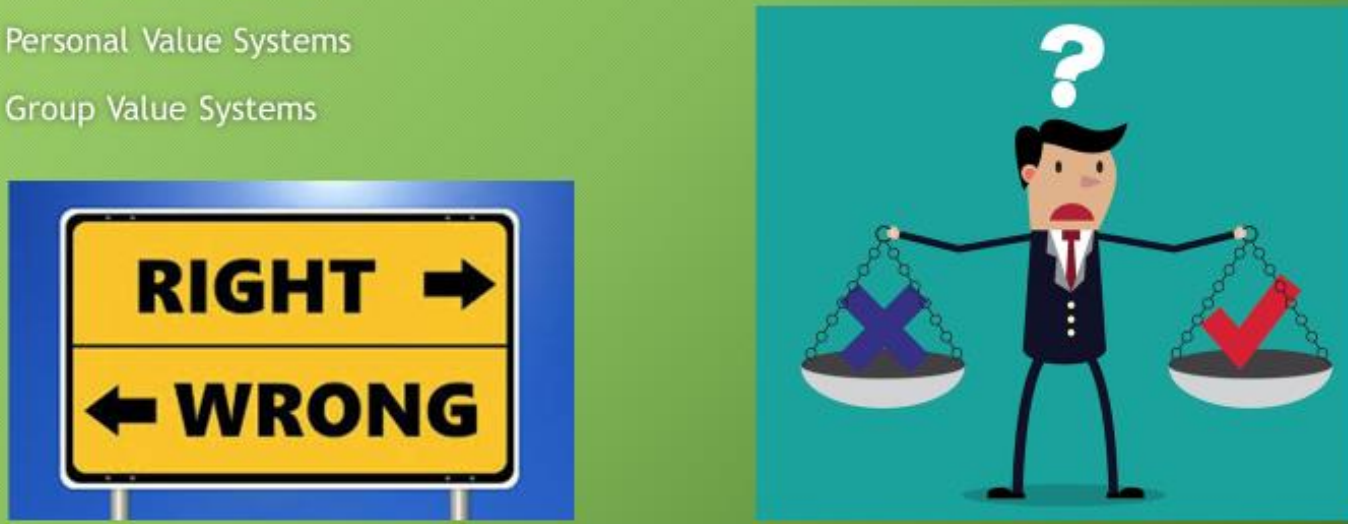

\section{Key Influences}

- Institutional Objectives

- Institutional Size

- Student-Faculty Relationships

- Curriculum

- Teaching

- Friendships and Student Committees

- Student Development Programs and Services 


\section{Recap}

- Seven Vectors

- Seven Key Influences 


\section{Appendix I \\ Standard Online Lesson Script (Control) \\ [The instructor shares their screen, displaying a PowerPoint presentation of an Introduction slide with the title "College Student Development."]}

Hello, everyone. My name is [INSTRUCTOR NAME], and today's lesson is called "College Student Development." Thank you all for tuning in today! This lesson is part of a study that I am conducting for my dissertation, so I really appreciate you all taking the time to join me today.

In this lesson, we will be talking about what we know as far as how students like you develop a sense of identity throughout your college experiences, as well as identify some different environmental factors that can affect exactly how that happens. Let's get started!

\section{[A new PowerPoint slide (slide 2) appears on the screen (see PowerPoint attachment).]}

In 1969, a researcher named Arthur Chickering developed a theory to describe how students discover who they are during college.

Even though identity and identity development are important topics for a person at any point during their lives, Chickering believed that establishing identity is a core developmental issue that people especially grapple with during their time in college. In fact, Chickering proposed that there are actually seven different vectors of development that each contribute to how college students' form their individual identities and sense of self - and he argued that these seven vectors can encompass different emotional, interpersonal, ethical, and intellectual aspects of who we are.

These vectors, specifically, are: (1) Developing Competence, (2) Managing Emotions, (3) Moving Through Autonomy Toward Interdependence, (4) Developing Mature Interpersonal Relationships, (5) Establishing Identity, (6) Developing Purpose, and (7) Developing Integrity.

Chickering described the seven vectors as "highways for journeying toward individuation." In other words, the seven vectors are different ways in which college students develop a sense of self and an understanding of who they are. The reason that Chickering labeled each of these as vectors is because identity development isn't necessarily a straight line or a step-by-step process.

In fact, we can actually progress or digress across each of these vectors with different intensity at different points in time - essentially moving up or down, left or right, forwards or backwards, or really in whatever direction our college experiences end up taking us. 
For example, just because you might have already established a sense of identity before you started college doesn't mean that your identity hasn't changed since your first semester. Instead, it's always more or less in a state of flux as you try new things, learn new information, and meet new people.

To make things even messier, Chickering suggested that college students can move through the seven vectors at different speeds, might have to deal with issues related to more than one vector at the same time, that vectors can interact with one another, and that college students frequently end up reexamining issues associated with vectors they may have previously resolved. Long story short, the ways that college students develop a sense of identity can get pretty complicated, and how well college students are able to navigate those complications can have a huge impact on how they ultimately come to see themselves as individuals.

Let's talk about each of Chickering's seven vectors of college student development in a little more detail to really flesh out what he was talking about.

\section{[A new PowerPoint slide (slide 3) appears on the screen (see PowerPoint attachment).]}

The first vector that Chickering identified is the Developing Competence vector.

Competence is all about feeling confident - it's the belief that you have what it takes to overcome obstacles and achieve your goals. Chickering suggested that college students might feel more or less competent in different settings, or based on what they are specifically trying to do at a particular point in time. In other words, Chickering didn't believe that someone is just competent all the time regardless of what they're doing, where they're at, or who they're with. Instead, he argued that there are three different types of competence that college students develop throughout their undergraduate experiences: intellectual competence, physical competence, and interpersonal competence.

Chickering characterized a person's competence as a pitchfork with three tines, and he said that intellectual, physical, and interpersonal competence are each three metaphorical tines on the pitchfork. When college students develop intellectual, physical, and interpersonal competence - basically sharpening each tine of the pitchfork - their overall competence is enhanced.

Intellectual competence is the first tine on the pitchfork, and it basically involves learning new things. Intellectual competence is all about acquiring knowledge and skills related to a particular subject, or developing the ability to think critically and engage in complex reasoning. Intellectual competence, in a sense, is the reason you take a lot of the courses that you do during college - to develop knowledge about whatever the subject of a particular course is. 
Physical competence, the second tine on Chickering's metaphorical pitchfork, refers to the development of manual skills or the ability to "do" something. Physical competence is closely related to athletic and recreational activities, attention to wellness, or involvement in artistic and manual activities. Playing a sport, exercising, painting, playing music each of these is an example of physical competence because you have to develop some level of physical skill in order to do them.

The third and final tine on the pitchfork is interpersonal competence, which is all about having the ability to communicate with others, be a leader, and work effectively in a team. If someone has developed interpersonal competence, it means that they can convey their thoughts and feelings to others effectively and appropriately. Do you know anyone who's able to make new friends easily? If you do, chances are that person is high in interpersonal competence - or, in other words, they have strong social skills.

\section{[A new PowerPoint slide (slide 4) appears on the screen (see PowerPoint attachment).]}

Chickering's second vector of college student development is Managing Emotions. In the Managing Emotions vector, college students develop the ability to recognize and accept their own emotions, as well as the emotions of others. Just as important, it is in the Managing Emotions vector that we learn how to express our emotions appropriately.

You don't need me to tell you that college can be pretty chaotic. College students are dealing with constant changes personally, academically, and professionally all at once. These changes, in turn, can make us feel a lot of different ways. Sometimes things happen that leave us feeling positive emotions like excitement, hope, or inspiration.

Think of a time when maybe you did well on an exam when you weren't expecting to, or when you finally got a phone number from someone you were interested in romantically. When things are going well, it's good to take a moment to appreciate the positive emotions that we experience.

In contrast, sometimes things don't go our way and we experience more negative emotions like anxiety, depression, anger, or shame.

Plenty of things can go wrong in college. Maybe you've done something at a party that backfired and left you feeling embarrassed, or maybe you've been having problems in a relationship that is important to you as you navigate the challenges of a college environment. College is often described as just being a stressful time in general, and maybe you've been having a tough time handling that stress. Just like it's important to recognize when we're feeling positive, acknowledging our negative emotions is an important part of the Managing Emotions vector.

Different emotions can influence behavior in different ways, and we need to develop the ability to express our emotions constructively or restrain ourselves from engaging in emotional behavior that could negatively impact ourselves or the people around us. 
Essentially, the Managing Emotions vector is all about learning how to feel and how to act on those feelings.

\section{[A new PowerPoint slide (slide 5) appears on the screen (see PowerPoint attachment).]}

The third vector is Moving Through Autonomy Toward Interdependence. For most students, the transition to a college or university environment is accompanied by a sense personal freedom that they have never really experienced before.

As students move away from home and begin living on their own, they become more independent. They learn to stand on their own two feet and they're able to make important decisions for themselves, rather than relying on instructions from a parental figure.

As students experience and enjoy their newfound autonomy, they also develop new relationships with other college students enjoying their own independence.

As they grow closer, they create new groups - keeping their independence as individuals while simultaneously forming connections with others and learning to rely on one another.

The Moving Through Autonomy Toward Interdependence vector is all about college students coming to terms with the fact that they are both independent from and interdependent with others at the same time. In other words, we're self-sufficient individuals and members of larger groups simultaneously, and our actions affect other members of the groups that we belong to.

\section{[A new PowerPoint slide (slide 6) appears on the screen (see PowerPoint attachment).]}

Chickering's fourth vector is the Developing Mature Interpersonal Relationships vector. In this vector, Chickering suggested that college students develop tolerance and appreciation for intercultural and interpersonal differences, as well as the capacity for long-lasting and healthy relationships with others. In the Developing Mature Interpersonal Relationships vector, college students recognize that not everyone thinks, feels, looks, or lives the same way they do. They develop the ability to accept others for who they are, respect ways in which others are different, and appreciate areas of commonality wherever they may exist.

As an example, the Developing Mature Interpersonal Relationships vector played a huge part in my development as a college student. I grew up in a really small town where, for the most part, people were more similar than they were different. Everyone tended to dress the same, listen to the same music, have the same hobbies, have similar perspectives and beliefs, and there wasn't too much diversity. When I went to college, it was actually really jarring - in a good way - to meet people from many different walks of 
life. I met people with different religious beliefs, people from different cultural backgrounds, people with different sexual identities, and people with different political beliefs.

It provided me with awesome opportunities to think in ways that I never had before, exploring new ideas, new interests, and new ways of seeing the world. Sometimes we are instinctively tempted to close ourselves off from people who are different than us, but resisting that temptation is extremely important if we're going to progress through the Developing Mature Interpersonal Relationships vector.

\section{[A new PowerPoint slide (slide 7) appears on the screen (see PowerPoint attachment).]}

The fifth vector is Establishing Identity. Chickering suggested that identity is extremely multifaceted - in other words, it's made up of a lot of different parts, or pieces. Identity includes your comfort with your body and appearance, your gender and sexual orientation, your social or cultural heritage, your self-concept and self-esteem, and your sense of personal stability and integration. Given this, Chickering argued that our progress in the Establishing Identity vector is actually based on the four vectors that we've already discussed in this lesson: Developing Competence, Managing Emotions, Moving Through Autonomy Toward Interdependence, and Developing Mature Interpersonal Relationships.

Each of these four vectors is basically a smaller piece of a puzzle that all come together in the Establishing Identity vector, creating a fuller, more complete picture of who we are.

\section{[A new PowerPoint slide (slide 8) appears on the screen (see PowerPoint attachment).]}

The sixth vector is Developing Purpose. In the Developing Purpose vector, you basically figure out why you get up in the morning. This vector involves college students identifying and pursuing goals. Those goals can be vocational and career-oriented, or they might involve meaningful commitments to personal interests or things you enjoy. They might also be based on interpersonal commitments you've made or are in the process of making, like your obligations to new friends or a new significant other. The Developing Purpose vector is focused on asking yourself, "why?"

When Developing Purpose, college students attempt to find direction for themselves in life, asking themselves questions such as "Why am I here?" In a sense, the Developing Purpose vector is all about finding the reason that you're here, why you do what you do, and why you get out of bed each morning.

\section{[A new PowerPoint slide (slide 9) appears on the screen (see PowerPoint attachment).]}


The seventh and final vector of Chickering's theory is the Developing Integrity vector. The Developing Integrity vector involves us recognizing that our moral and ethical values have an effect on our actions. Chickering suggested that college students progress through the Developing Integrity vector as they transition from making decisions based on rigid, moralistic thinking to systems of problem-solving and decision-making that strike a balance between self-interest and social responsibility. Basically, college students move through the Developing Integrity vector as they learn to balance their personal value systems with the value systems held by the groups that they belong to.

As we grow up, we are often told what is "right" or "wrong," and the world can come off to us as a bit black and white. We're often told that lying, for example, is always wrong and that we should always strive to be honest. As we move through the Developing Integrity vector, we start to re-examine ideas like this. What if the lie protects someone who we care about? What if the lie spares someone's feelings? What if the lie is intended to be helpful? Imagine someone asks you if you've been planning a surprise party for their birthday - do you spoil the surprise and ruin the birthday party for the sake of being honest?

As college students, you've probably already found yourselves in plenty of situations that have challenged your pre-existing ideas of right and wrong. Another reason that college is such an important time for our identity development is that it forces us into situations where we begin to realize that the world is not as black and white as we might have originally thought.

Right and wrong are not always easy to distinguish from each other, and during college many students start to recognize and appreciate this.

\section{[A new PowerPoint slide (slide 10) appears on the screen (see PowerPoint attachment).]}

Chickering argued that how college students develop their identities across the seven vectors we just discussed is heavily influenced by their college or university environment. In particular, he suggested that there are seven key environmental factors that can influence college students' identity development - factors that he described as key influences. Let's discuss each of these seven key influences briefly:

The first of the seven key influences that Chickering identified is Institutional Objectives. Institutional Objectives refer to the goals of a particular college or university. A lot of schools have a mission statement - an official articulation of the values or ideas that are important to them. Basically, think of this like a school's motto.

Not too surprisingly, this can lead different schools to prioritize different aspects of their students' identity development. For example, while some colleges or universities might prioritize developing students' competence - especially their intellectual competence others might pay more attention to developing students' sense of integrity and ability to discern right from wrong. The vectors of identity development that a school identifies as 
most important in their Institutional Objectives can really influence the ways in which that school sets up its programs. As a result, it can have a huge impact on how students who attend a particular school develop their individual identities.

The second key influence is Institutional Size. Chickering proposed that student participation in college life and satisfaction with the college experience go hand-in-hand, and college students' experiences may be diminished at larger colleges and universities. Students' progression across the seven vectors of identity development requires meaningful participation in opportunities provided by a college or university, which Chickering warned may not necessarily be provided at institutions with excessively high numbers of students. Larger schools typically offer programs, events, and other opportunities that cast a wide net - they are designed to be generally applicable to as many students as possible. For example, most people like pizza, so a large school might have a pizza night in the student commons. In contrast, Chickering suggested that smaller institutions are more likely to provide students with opportunities that are more customtailored to students' specific interests, needs, and goals. For example, maybe a small school that is primarily oriented towards the arts and humanities hosts an independent film contest or an art gallery for exhibiting students' work. With less students, smaller schools can offer pretty specific opportunities rather than opportunities that are one-sizefits-all.

The third key influence is Student-Faculty Relationships. Chickering suggested that meaningful interaction between students and faculty plays a huge part in students' identity development. Because of this, he argued that students should be able to see their teachers in a variety of situations involving different roles and responsibilities rather than exclusively during class. As students begin to perceive faculty as real human beings who are genuinely interested in who they are and are accessible outside of the classroom, students and faculty receive opportunities to learn more about one another as individuals and create deeper personal, professional, and academic relationships that can enhance students' identity development. Basically, there's only so much that your teachers can learn about you during class. If you interact outside of class, you can create a meaningful relationship and, as they learn more about you, maybe they can provide you with unique opportunities you'd have never even heard about otherwise.

The fourth key influence is Curriculum. Chickering believed that the curriculum in the classes that each of you are taking has a tremendous influence on your identity development. Specifically, he argued that curriculum needs to be relevant to your interests, your needs, your goals, and your experiences, as well as offer diverse perspectives that represent a variety of values and beliefs. Have you ever taken a class just because you needed a certain type of course credit - like a general elective - and found yourself wondering "What the heck does this topic have to do with my life?" According to Chickering, that should never happen - the curriculum in your classes should be curriculum that you can actually use, not information that you forget and never need to apply again after a semester ends. 
The fifth key influence is Teaching. Chickering suggested that students' learning - and ultimately identity development - occurs when teachers at colleges and universities incorporate active learning, ongoing interaction with students inside and outside of class, timely and specific feedback, high expectations, and respect for individual learning differences into their instruction. In these ways, Chickering proposed that teachers can stimulate college students' progression across multiple vectors of identity development at the same time rather than focusing exclusively on students' intellectual competence. In other words, when your students understand the ways in which you learn, teach in those ways, and engage you in the learning process, your identity development will be enhanced.

The sixth key influence is Friendships and Student Communities. Chickering once wrote that "a student's most important teacher is often another student." Basically, Chickering proposed that college students' development across all seven vectors is enhanced when students form meaningful friendships and participate in diverse student communities characterized by shared interests and ongoing interactions. Communities that college students participate in can be informal groups, like a circle of friends, or formal groups like residence hall floors, sports teams, student organizations, or classes. Communities that stimulate college students' development typically encourage regular communication and interaction between students, offer students opportunities to work together, are small and intimate enough so that no one feels redundant or left out, and are comprised of individuals from diverse backgrounds.

The seventh and final key influence is Student Development Programs and Services. Chickering argued that it is important for faculty and student affairs professionals at colleges and universities to provide programs and services specifically designed to assist students in exploring and developing their individual identities. Rather than focusing solely on students' performance in the classroom, Chickering proposed that "educators" should instead refer to themselves as "student development professionals" and serve as advocates for the development of the whole student. By providing formal programs strategically designed to assist students' self-exploration and development, faculty and staff can provide valuable assistance to college students' progression across all seven vectors of identity development.

\section{[A new PowerPoint slide (slide 11) appears on the screen (see PowerPoint attachment).]}

And that will do it for the seven key influences!

So, to quickly recap - in this lesson, we discussed Arthur Chickering's theory of college student development, as well as identified different institutional factors which can influence the ways in which college students' identity development takes place. In particular, we reviewed Chickering's seven vectors of identity development and the seven key influences, or environmental factors, which he argued can influence the extent to which college students' identity development occurs. 
Thank you again for participating in this lesson!

If you are seeking extra credit in a Communication Studies course for participating in this lesson, you will find a hyperlink in the Zoom chat to a brief survey that will ask you questions related to the lesson that you just participated in. Once you complete the survey, you will be provided with an additional link which will take you to a separate extra credit research receipt survey.

[The instructor posts a link to the post-lesson survey in the Zoom Chat.]

https://wvu.qualtrics.com/jfe/form/SV_6WHvx4gprQcEEBM

Have a great day, everyone - and thank you again!

[The instructor disables screen-sharing. After five minutes, the instructor closes the Zoom lesson.] 
Appendix $\mathbf{J}$

CTML-based Online Lesson Script (Treatment)

[The instructor shares their screen, displaying a PowerPoint presentation of an Introduction slide with the title "College Student Development." A live video image of the instructor is visible in the upper right corner of the screen.]

Hello, everyone. My name is [INSTRUCTOR NAME], and today's lesson is called "College Student Development." Thank you all for tuning in today! This lesson is part of a study that I am conducting for my dissertation, so I really appreciate you all taking the time to join me today.

In this lesson, we will be talking about what we know as far as how students like you develop a sense of identity throughout your college experiences, as well as identify some different environmental factors that can affect exactly how that happens. Let's get started!

\section{[A new PowerPoint slide (slide 2) appears on the screen (see PowerPoint attachment).]}

In 1969, a researcher named Arthur Chickering developed a theory to describe how students discover who they are during college.

[The instructor draws a circle around the name "Arthur Chickering" on the screen as he says it.]

Even though identity and identity development are important topics for a person at any point during their lives, Chickering believed that establishing identity is a core developmental issue that people especially grapple with during their time in college. In fact, Chickering proposed that there are actually seven different vectors of development that each contribute to how college students' form their individual identities and sense of self - and he argued that these seven vectors can encompass different emotional, interpersonal, ethical, and intellectual aspects of who we are.

These vectors, specifically, are: (1) Developing Competence, (2) Managing Emotions, (3) Moving Through Autonomy Toward Interdependence, (4) Developing Mature Interpersonal Relationships, (5) Establishing Identity, (6) Developing Purpose, and (7) Developing Integrity.

[The instructor says and types the appropriate number next to each vector as he reads the name of the vector aloud (e.g., the instructor says and types " 1 " beside "Developing Competence" on the screen as he names the vector).]

Chickering described the seven vectors as "highways for journeying toward individuation." In other words, the seven vectors are different ways in which college students develop a sense of self and an understanding of who they are. The reason that 
Chickering labeled each of these as vectors is because identity development isn't necessarily a straight line or a step-by-step process.

\section{[The instructor underlines the word "vector," then draws a red "X" across the image of a straight arrow presented on the power point slide.]}

In fact, we can actually progress or digress across each of these vectors with different intensity at different points in time - essentially moving up or down, left or right, forwards or backwards, or really in whatever direction our college experiences end up taking us.

[The instructor draws a green checkmark in the white space included in the image of the multi-directional arrow.]

For example, just because you might have already established a sense of identity before you started college doesn't mean that your identity hasn't changed since your first semester. Instead, it's always more or less in a state of flux as you try new things, learn new information, and meet new people.

To make things even messier, Chickering suggested that college students can move through the seven vectors at different speeds, might have to deal with issues related to more than one vector at the same time, that vectors can interact with one another, and that college students frequently end up reexamining issues associated with vectors they may have previously resolved. Long story short, the ways that college students develop a sense of identity can get pretty complicated, and how well college students are able to navigate those complications can have a huge impact on how they ultimately come to see themselves as individuals.

Let's talk about each of Chickering's seven vectors of college student development in a little more detail to really flesh out what he was talking about.

[The instructor removes all markings from the screen. A new PowerPoint slide (slide 3) appears on the screen (see PowerPoint attachment).]

The first vector that Chickering identified is the Developing Competence vector.

[The instructor "1" next to the words "Developing Competence" on the PowerPoint slide.]

Competence is all about feeling confident - it's the belief that you have what it takes to overcome obstacles and achieve your goals. Chickering suggested that college students might feel more or less competent in different settings, or based on what they are specifically trying to do at a particular point in time. In other words, Chickering didn't believe that someone is just competent all the time regardless of what they're doing, where they're at, or who they're with. Instead, he argued that there are three different types of competence that college students develop throughout their undergraduate 
experiences: intellectual competence, physical competence, and interpersonal competence.

[The instructor draws a blue dot beside "intellectual competence" as he says it aloud, an orange dot beside "physical competence" as he says it aloud, and a purple dot beside "interpersonal competence" as he says it aloud.]

Chickering characterized a person's competence as a pitchfork with three tines, and he said that intellectual, physical, and interpersonal competence are each three metaphorical tines on the pitchfork. When college students develop intellectual, physical, and interpersonal competence - basically sharpening each tine of the pitchfork - their overall competence is enhanced.

[The instructor draws a blue line - the same color as the dot located beside "intellectual competence" - along the leftmost tine in the image of the pitchfork.]

Intellectual competence is the first tine on the pitchfork, and it basically involves learning new things. Intellectual competence is all about acquiring knowledge and skills related to a particular subject, or developing the ability to think critically and engage in complex reasoning. Intellectual competence, in a sense, is the reason you take a lot of the courses that you do during college - to develop knowledge about whatever the subject of a particular course is.

[The instructor draws an orange line - the same color as the dot located beside "physical competence" - along the middle tine in the image of the pitchfork.]

Physical competence, the second tine on Chickering's metaphorical pitchfork, refers to the development of manual skills or the ability to "do" something. Physical competence is closely related to athletic and recreational activities, attention to wellness, or involvement in artistic and manual activities. Playing a sport, exercising, painting, playing music each of these is an example of physical competence because you have to develop some level of physical skill in order to do them.

[The instructor draws a purple line - the same color as the dot located beside "interpersonal competence" - along the rightmost tine in the image of the pitchfork.]

The third and final tine on the pitchfork is interpersonal competence, which is all about having the ability to communicate with others, be a leader, and work effectively in a team. If someone has developed interpersonal competence, it means that they can convey their thoughts and feelings to others effectively and appropriately. Do you know anyone who's able to make new friends easily? If you do, chances are that person is high in interpersonal competence - or, in other words, they have strong social skills.

So, before we move any further, let's make sure we understand what we have covered so far with a quick Zoom poll. 
I'm sure that most of you have had at least one experience during college where you needed to complete a task in a group. Think back on your group experience and answer this question for me: which of the three types of competence we just discussed is most closely related to how we work together with others in groups?

[The instructor activates a Zoom poll. The prompt for the poll reads "Which of the three types of competence we just discussed is most closely related to how we work together with others in groups?" There are three responses to choose from: "Intellectual Competence," "Physical Competence," and "Interpersonal Competence." The instructor waits for fifteen seconds to allow students to read the prompt and select a response. After the fifteen seconds pass, the instructor closes the poll. The instructor shares the poll results and reveals the correct answer, "Interpersonal Competence."]

Thanks for answering that - and those of you who chose "Interpersonal Competence," great job! Even though it's possible that you could be have developed your Intellectual and Physical Competence while working together with your group, actually learning how to work together with others effectively is definitely most closely aligned with "Interpersonal Competence."

Okay, let's move on to our next vector.

[The instructor removes all markings from the screen. A new PowerPoint slide (slide 4) appears on the screen (see PowerPoint attachment). The instructor types "2" next to the words "Managing Emotions" on the PowerPoint slide.]

Chickering's second vector of college student development is Managing Emotions. In the Managing Emotions vector, college students develop the ability to recognize and accept their own emotions, as well as the emotions of others. Just as important, it is in the Managing Emotions vector that we learn how to express our emotions appropriately.

You don't need me to tell you that college can be pretty chaotic. College students are dealing with constant changes personally, academically, and professionally all at once. These changes, in turn, can make us feel a lot of different ways. Sometimes things happen that leave us feeling positive emotions like excitement, hope, or inspiration.

[The instructor draws a circle around the image of the green smiling face in the image included in the PowerPoint.]

Think of a time when maybe you did well on an exam when you weren't expecting to, or when you finally got a phone number from someone you were interested in romantically. When things are going well, it's good to take a moment to appreciate the positive emotions that we experience. 
In contrast, sometimes things don't go our way and we experience more negative emotions like anxiety, depression, anger, or shame.

[The instructor removes the circle around and the green smiling face and draws a new circle around the image of the red frowning face in the image included in the PowerPoint.]

Plenty of things can go wrong in college. Maybe you've done something at a party that backfired and left you feeling embarrassed, or maybe you've been having problems in a relationship that is important to you as you navigate the challenges of a college environment. College is often described as just being a stressful time in general, and maybe you've been having a tough time handling that stress. Just like it's important to recognize when we're feeling positive, acknowledging our negative emotions is an important part of the Managing Emotions vector.

Different emotions can influence behavior in different ways, and we need to develop the ability to express our emotions constructively or restrain ourselves from engaging in emotional behavior that could negatively impact ourselves or the people around us. Essentially, the Managing Emotions vector is all about learning how to feel and how to act on those feelings.

[The instructor removes all markings from the screen. A new PowerPoint slide (slide 5) appears on the screen (see PowerPoint attachment). The instructor types "3" next to the words "Moving Through Autonomy Toward Interdependence" on the PowerPoint slide.]

The third vector is Moving Through Autonomy Toward Interdependence. For most students, the transition to a college or university environment is accompanied by a sense personal freedom that they have never really experienced before.

[The instructor uses draws a rectangle around the single stick figure in the image presented on the PowerPoint slide.]

As students move away from home and begin living on their own, they become more independent. They learn to stand on their own two feet and they're able to make important decisions for themselves, rather than relying on instructions from a parental figure.

As students experience and enjoy their newfound autonomy, they also develop new relationships with other college students enjoying their own independence.

[The instructor draws a brighter arrow over the one already displayed in the image presented on the PowerPoint slide while saying "they also develop new relationships with other college students enjoying their own newfound independence."] 
As they grow closer, they create new groups - keeping their independence as individuals while simultaneously forming connections with others and learning to rely on one another.

[The instructor draws a rectangle around the group of stick figures in the image presented on the PowerPoint slide while saying "create new groups."]

The Moving Through Autonomy Toward Interdependence vector is all about college students coming to terms with the fact that they are both independent from and interdependent with others at the same time. In other words, we're self-sufficient individuals and members of larger groups simultaneously, and our actions affect other members of the groups that we belong to.

[The instructor removes all markings from the screen. A new PowerPoint slide (slide 6) appears on the screen (see PowerPoint attachment). The instructor types "4" next to the words "Developing Mature Interpersonal Relationships" on the PowerPoint slide.]

Chickering's fourth vector is the Developing Mature Interpersonal Relationships vector. In this vector, Chickering suggested that college students develop tolerance and appreciation for intercultural and interpersonal differences, as well as the capacity for long-lasting and healthy relationships with others. In the Developing Mature Interpersonal Relationships vector, college students recognize that not everyone thinks, feels, looks, or lives the same way they do. They develop the ability to accept others for who they are, respect ways in which others are different, and appreciate areas of commonality wherever they may exist.

As an example, the Developing Mature Interpersonal Relationships vector played a huge part in my development as a college student. I grew up in a really small town where, for the most part, people were more similar than they were different. Everyone tended to dress the same, listen to the same music, have the same hobbies, have similar perspectives and beliefs, and there wasn't too much diversity. When I went to college, it was actually really jarring - in a good way - to meet people from many different walks of life. I met people with different religious beliefs, people from different cultural backgrounds, people with different sexual identities, and people with different political beliefs.

[The instructor uses the draw feature to create an arrow pointing to the blue stick figure while saying "different religious beliefs," to the red stick figure while saying "different cultural backgrounds," to the purple stick figure while saying "different sexual identities," and to the green stick figure while saying "different political beliefs."]

It provided me with awesome opportunities to think in ways that I never had before, exploring new ideas, new interests, and new ways of seeing the world. Sometimes we are instinctively tempted to close ourselves off from people who are different than us, but 
resisting that temptation is extremely important if we're going to progress through the Developing Mature Interpersonal Relationships vector.

[The instructor removes all markings from the screen. A new PowerPoint slide (slide 7) appears on the screen (see PowerPoint attachment). The types " 5 " next to the words "Establishing Identity" on the PowerPoint slide.]

The fifth vector is Establishing Identity. Chickering suggested that identity is extremely multifaceted - in other words, it's made up of a lot of different parts, or pieces. Identity includes your comfort with your body and appearance, your gender and sexual orientation, your social or cultural heritage, your self-concept and self-esteem, and your sense of personal stability and integration. Given this, Chickering argued that our progress in the Establishing Identity vector is actually based on the four vectors that we've already discussed in this lesson: Developing Competence, Managing Emotions, Moving Through Autonomy Toward Interdependence, and Developing Mature Interpersonal Relationships.

[While reading the name of each vector, the instructor uses the draw feature to draw arrows from each to a specific puzzle piece in the image presented on the PowerPoint slide.]

Each of these four vectors is basically a smaller piece of a puzzle that all come together in the Establishing Identity vector, creating a fuller, more complete picture of who we are.

[The instructor removes all markings from the screen. A new PowerPoint slide (slide 8) appears on the screen (see PowerPoint attachment). The instructor types "6" next to the words "Developing Purpose" on the PowerPoint slide.]

The sixth vector is Developing Purpose. In the Developing Purpose vector, you basically figure out why you get up in the morning. This vector involves college students identifying and pursuing goals. Those goals can be vocational and career-oriented, or they might involve meaningful commitments to personal interests or things you enjoy. They might also be based on interpersonal commitments you've made or are in the process of making, like your obligations to new friends or a new significant other. The Developing Purpose vector is focused on asking yourself, "why?"

[The instructor types "WHY?" in the center of the screen while saying "The Developing Purpose vector is focused on asking yourself, 'why?"'.]

When Developing Purpose, college students attempt to find direction for themselves in life, asking themselves questions such as "Why am I here?" In a sense, the Developing Purpose vector is all about finding the reason that you're here, why you do what you do, and why you get out of bed each morning. 
[The instructor removes all markings from the screen. A new PowerPoint slide (slide 9) appears on the screen (see PowerPoint attachment). The instructor types "7" next to the words "Developing Integrity."]

The seventh and final vector of Chickering's theory is the Developing Integrity vector. The Developing Integrity vector involves us recognizing that our moral and ethical values have an effect on our actions. Chickering suggested that college students progress through the Developing Integrity vector as they transition from making decisions based on rigid, moralistic thinking to systems of problem-solving and decision-making that strike a balance between self-interest and social responsibility. Basically, college students move through the Developing Integrity vector as they learn to balance their personal value systems with the value systems held by the groups that they belong to.

[The instructor draws an arrow from the text "Personal Value Systems" to one of the scales included in the rightmost image presented on the PowerPoint slide. The instructor uses the draw feature to create an arrow from the text "Group Value Systems" to the other side of the scale included in the rightmost image presented on the PowerPoint slide.]

As we grow up, we are often told what is "right" or "wrong," and the world can come off to us as a bit black and white. We're often told that lying, for example, is always wrong and that we should always strive to be honest. As we move through the Developing Integrity vector, we start to re-examine ideas like this. What if the lie protects someone who we care about? What if the lie spares someone's feelings? What if the lie is intended to be helpful? Imagine someone asks you if you've been planning a surprise party for their birthday - do you spoil the surprise and ruin the birthday party for the sake of being honest?

As college students, you've probably already found yourselves in plenty of situations that have challenged your pre-existing ideas of right and wrong. Another reason that college is such an important time for our identity development is that it forces us into situations where we begin to realize that the world is not as black and white as we might have originally thought.

[The instructor uses the draw feature to draw a red $X$ through the image of the sign displaying "Right $\rightarrow, \leftarrow$ Wrong".]

Right and wrong are not always easy to distinguish from each other, and during college many students start to recognize and appreciate this.

So, that wraps up Chickering's seven vectors.

Before we go any further, let's double-check that we understand what we have covered so far with another quick Zoom poll. 
I mentioned that one of the seven vectors we discussed, Establishing Identity, can be particularly complicated because it's based on how we develop across four other vectors. Take a moment and think back as best you can about which four vectors we said were the puzzle pieces that contribute to our development in the Establishing Identity vector. Then, tell me which four vectors influence how we establish our identity on this Zoom poll. Be sure to select all four vectors that you think apply.

[The instructor activates a class poll. The prompt for the poll reads "How we develop in the Establishing Identity vector is based on our development in which four other vectors? (Select all that apply)." There are six responses to choose from: (1) "Developing Competence", (2) "Managing Emotions", (3) "Moving Through Autonomy Toward Interdependence", (4) "Developing Mature Interpersonal Relationships", (5) "Developing Purpose", and (6) "Developing Integrity". The instructor waits for fifteen seconds to allow students to read the prompt and select a response. After the fifteen seconds pass, the instructor closes the poll. The instructor shares the poll results and reveals the correct answers.]

Thanks for answering that question - like I said, this one's complicated, so no worries if you might have answered wrong!

Like we discussed, the ways that we develop in the Establishing Identity vector is based on the first four vectors we covered in this lesson: Developing Competence, Managing Emotions, Moving Through Autonomy Toward Interdependence, and Developing Mature Interpersonal Relationships. These four vectors are essentially puzzle pieces that fit together as we progress through the Establishing Identity vector.

Alright, moving on...

[A new PowerPoint slide (slide 10) appears on the screen (see PowerPoint attachment).]

Chickering argued that how college students develop their identities across the seven vectors we just discussed is heavily influenced by their college or university environment. In particular, he suggested that there are seven key environmental factors that can influence college students' identity development - factors that he described as key influences. Let's discuss each of these seven key influences briefly:

[The instructor uses the annotation feature to type "1" beside "Institutional Objectives."]

The first of the seven key influences that Chickering identified is Institutional Objectives. Institutional Objectives refer to the goals of a particular college or university. A lot of schools have a mission statement - an official articulation of the values or ideas that are important to them. Basically, think of this like a school's motto. 
Not too surprisingly, this can lead different schools to prioritize different aspects of their students' identity development. For example, while some colleges or universities might prioritize developing students' competence - especially their intellectual competence others might pay more attention to developing students' sense of integrity and ability to discern right from wrong. The vectors of identity development that a school identifies as most important in their Institutional Objectives can really influence the ways in which that school sets up its programs. As a result, it can have a huge impact on how students who attend a particular school develop their individual identities.

\section{[The instructor removes all markings from the screen. The instructor uses the annotation feature to type "2" beside "Institutional Size."]}

The second key influence is Institutional Size. Chickering proposed that student participation in college life and satisfaction with the college experience go hand-in-hand, and college students' experiences may be diminished at larger colleges and universities. Students' progression across the seven vectors of identity development requires meaningful participation in opportunities provided by a college or university, which Chickering warned may not necessarily be provided at institutions with excessively high numbers of students. Larger schools typically offer programs, events, and other opportunities that cast a wide net - they are designed to be generally applicable to as many students as possible. For example, most people like pizza, so a large school might have a pizza night in the student commons. In contrast, Chickering suggested that smaller institutions are more likely to provide students with opportunities that are more customtailored to students' specific interests, needs, and goals. For example, maybe a small school that is primarily oriented towards the arts and humanities hosts an independent film contest or an art gallery for exhibiting students' work. With less students, smaller schools can offer pretty specific opportunities rather than opportunities that are one-sizefits-all.

\section{[The instructor removes all markings from the screen. The instructor uses the annotation feature to type " 3 " beside "Student-Faculty Relationships."]}

The third key influence is Student-Faculty Relationships. Chickering suggested that meaningful interaction between students and faculty plays a huge part in students' identity development. Because of this, he argued that students should be able to see their teachers in a variety of situations involving different roles and responsibilities rather than exclusively during class. As students begin to perceive faculty as real human beings who are genuinely interested in who they are and are accessible outside of the classroom, students and faculty receive opportunities to learn more about one another as individuals and create deeper personal, professional, and academic relationships that can enhance students' identity development. Basically, there's only so much that your teachers can learn about you during class. If you interact outside of class, you can create a meaningful relationship and, as they learn more about you, maybe they can provide you with unique opportunities you'd have never even heard about otherwise. 
[The instructor removes all markings from the screen. The instructor uses the annotation feature to type "4" beside "Curriculum."]

The fourth key influence is Curriculum. Chickering believed that the curriculum in the classes that each of you are taking has a tremendous influence on your identity development. Specifically, he argued that curriculum needs to be relevant to your interests, your needs, your goals, and your experiences, as well as offer diverse perspectives that represent a variety of values and beliefs. Have you ever taken a class just because you needed a certain type of course credit - like a general elective - and found yourself wondering "What the heck does this topic have to do with my life?" According to Chickering, that should never happen - the curriculum in your classes should be curriculum that you can actually use, not information that you forget and never need to apply again after a semester ends.

[The instructor removes all markings from the screen. The instructor uses the annotation feature to type "5" beside "Teaching."]

The fifth key influence is Teaching. Chickering suggested that students' learning - and ultimately identity development - occurs when teachers at colleges and universities incorporate active learning, ongoing interaction with students inside and outside of class, timely and specific feedback, high expectations, and respect for individual learning differences into their instruction. In these ways, Chickering proposed that teachers can stimulate college students' progression across multiple vectors of identity development at the same time rather than focusing exclusively on students' intellectual competence. In other words, when your students understand the ways in which you learn, teach in those ways, and engage you in the learning process, your identity development will be enhanced.

[The instructor removes all markings from the screen. The instructor uses the annotation feature to type "6" beside "Friendships and Student Committees."]

The sixth key influence is Friendships and Student Communities. Chickering once wrote that "a student's most important teacher is often another student." Basically, Chickering proposed that college students' development across all seven vectors is enhanced when students form meaningful friendships and participate in diverse student communities characterized by shared interests and ongoing interactions. Communities that college students participate in can be informal groups, like a circle of friends, or formal groups like residence hall floors, sports teams, student organizations, or classes. Communities that stimulate college students' development typically encourage regular communication and interaction between students, offer students opportunities to work together, are small and intimate enough so that no one feels redundant or left out, and are comprised of individuals from diverse backgrounds.

[The instructor removes all markings from the screen. The instructor uses the annotation feature to type "7" beside "Student Development Programs and Services."] 
The seventh and final key influence is Student Development Programs and Services. Chickering argued that it is important for faculty and student affairs professionals at colleges and universities to provide programs and services specifically designed to assist students in exploring and developing their individual identities. Rather than focusing solely on students' performance in the classroom, Chickering proposed that "educators" should instead refer to themselves as "student development professionals" and serve as advocates for the development of the whole student. By providing formal programs strategically designed to assist students' self-exploration and development, faculty and staff can provide valuable assistance to college students' progression across all seven vectors of identity development.

[The instructor removes all markings from the screen.]

Alright - so we moved through those seven key influences pretty fast compared to the seven vectors. Before we wrap up the lesson, let's make we are all on the same page with one last Zoom poll.

Each of you are already taking classes here at WVU, and those classes are taught by different instructors. According to Chickering, your identity development is enhanced when you are able to interact with those instructors inside and outside of the classroom because it allows you to learn more about each other as people. This idea is the main point behind which of the following key influences?

[The instructor activates a Zoom poll. The prompt for the poll reads "Which of the seven key influences involves students and instructors interacting both inside and outside of the classroom?" There are seven responses to choose from: (1) "Institutional Objectives," (2) "Institutional Size," (3) "Student-Faculty Relationships," (4) "Curriculum," (5) "Teaching," (6) "Friendships and Student Committees," and (7)"Student Development Programs and Services." The instructor waits for fifteen seconds to allow students to select a response. After the fifteen seconds pass, the instructor closes the poll. The instructor shares the poll results and reveals the correct answer, "Student-Faculty Relationships."]

Thanks for participating in that last poll, and great work if you chose "StudentFaculty Relationships!" Chickering argued that Student-Faculty Relationships play a huge part in how we develop a sense of identity during college, and he said that these relationships become more meaningful when we don't just see our instructors during class time.

[A new PowerPoint slide (slide 11) appears on the screen (see PowerPoint attachment).]

And that will do it for the seven key influences! 
So, to quickly recap - in this lesson, we discussed Arthur Chickering's theory of college student development, as well as identified different institutional factors which can influence the ways in which college students' identity development takes place. In particular, we reviewed Chickering's seven vectors of identity development and the seven key influences, or environmental factors, which he argued can influence the extent to which college students' identity development occurs.

Thank you again for participating in this lesson!

If you are seeking extra credit in a Communication Studies course for participating in this lesson, you will find a hyperlink in the Zoom chat to a brief survey that will ask you questions related to the lesson that you just participated in. Once you complete the survey, you will be provided with an additional link which will take you to a separate extra credit research receipt survey.

[The instructor posts a link to the post-lesson survey in the Zoom Chat.]

https://wvu.qualtrics.com/jfe/form/SV_2rU13RLlnt1m4sK

Have a great day, everyone - and thank you again!

[The instructor turns off his camera and disables screen-sharing. After five minutes, the instructor closes the Zoom lesson.] 


\section{Appendix K}

Selection Scale (Bolkan, 2018)

\begin{tabular}{|c|c|c|c|c|c|c|}
\hline $\begin{array}{c}\text { Strongly } \\
\text { Disagree }\end{array}$ & $\begin{array}{c}\text { Moderately } \\
\text { Disagree }\end{array}$ & $\begin{array}{c}\text { Slightly } \\
\text { Disagree }\end{array}$ & $\begin{array}{c}\text { Neither } \\
\text { Disagree } \\
\text { nor Agree }\end{array}$ & $\begin{array}{c}\text { Slightly } \\
\text { Agree }\end{array}$ & $\begin{array}{c}\text { Moderately } \\
\text { Agree }\end{array}$ & $\begin{array}{c}\text { Strongly } \\
\text { Agree }\end{array}$ \\
1 & 2 & 3 & 4 & 5 & 6 & 7 \\
\hline
\end{tabular}

1. I found it easy to identify the crucial aspects of this lesson.

2. I was able to recognize what information was most relevant to learning the material.

3. I had the ability to differentiate what was important to know in this lesson from what was not.

4. I could determine what was significant to pay attention to in this lesson.

5. I knew what aspects of this lesson to concentrate on.

6. I understood what I was supposed to focus on during this lesson. 
Appendix L

Organization Scale (Bolkan, 2017b)

\begin{tabular}{|c|c|c|c|c|c|c|}
\hline $\begin{array}{c}\text { Strongly } \\
\text { Disagree }\end{array}$ & $\begin{array}{c}\text { Moderately } \\
\text { Disagree }\end{array}$ & $\begin{array}{c}\text { Slightly } \\
\text { Disagree }\end{array}$ & $\begin{array}{c}\text { Neither } \\
\text { Disagree } \\
\text { nor Agree }\end{array}$ & $\begin{array}{c}\text { Slightly } \\
\text { Agree }\end{array}$ & $\begin{array}{c}\text { Moderately } \\
\text { Agree }\end{array}$ & $\begin{array}{c}\text { Strongly } \\
\text { Agree }\end{array}$ \\
1 & 2 & 3 & 4 & 5 & 6 & 7 \\
\hline
\end{tabular}

1. I was able to organize the material presented in this lesson in a logical manner.

2. I could connect the ideas in this lesson to one another in a coherent fashion.

3. I understood the relationships between the various parts of this lesson.

4. I had the ability to logically model concepts from this lesson as they applied to one another.

5. I grasped how the various parts of this lesson worked jointly to form the ideas I was learning. 


\section{Appendix M}

Working Memory Overload Subscale (Bolkan, 2017a)

\begin{tabular}{|c|c|c|c|c|c|c|}
\hline $\begin{array}{c}\text { Strongly } \\
\text { Disagree }\end{array}$ & $\begin{array}{c}\text { Moderately } \\
\text { Disagree }\end{array}$ & $\begin{array}{c}\text { Slightly } \\
\text { Disagree }\end{array}$ & $\begin{array}{c}\text { Neither } \\
\text { Disagree } \\
\text { nor Agree }\end{array}$ & $\begin{array}{c}\text { Slightly } \\
\text { Agree }\end{array}$ & $\begin{array}{c}\text { Moderately } \\
\text { Agree }\end{array}$ & $\begin{array}{c}\text { Strongly } \\
\text { Agree }\end{array}$ \\
1 & 2 & 3 & 4 & 5 & 6 & 7 \\
\hline
\end{tabular}

1. The amount of information presented in the lesson was overwhelming.

2. There was so much to learn during the lesson that I had a hard time keeping up.

3. I felt flustered trying to keep up with the amount of information presented in this lesson.

4. This lesson made me feel anxious because of the amount of information I was asked to learn all at one time. 


\section{Appendix N}

\section{Post-Lesson Test}

INSTRUCTIONS: This test consists of 10 questions that will assess your knowledge of college student development. Please select the best answer for each question. You will not be able to return to previous questions once you have provided a response, so please consider your answers carefully.

1. The Theory of College Student Development was created by
a) Stanley Milgram
b) Arthur Chickering
c) Albert Bandura
d) Lawrence Kohlberg

2. According to the Theory of College Student Development, there are vectors that students move through while developing their identities.
a) Three
b) Five
c) Seven
d) Nine

3. According to the Theory of College Student Development, there are key influences (i.e., environmental factors) that can affect college students' identity development.
a) Three
b) Five
c) Seven
d) Nine

4. The vector of college student development has been described as a three-tined pitchfork because it has three different dimensions.
a) Developing Competence
b) Managing Emotions
c) Establishing Identity
d) Developing Integrity

5. The vector of college student development is similar to a puzzle because it is based on college students' development across four other vectors: Developing Competence, Managing Emotions, Moving Through Autonomy Toward Interdependence, and Developing Mature Interpersonal Relationships.
a) Establishing Identity
b) Developing Integrity
c) Developing Purpose
d) Student-Faculty Relationships 
6. Developing tolerance and appreciation for peoples' differences (e.g., cultural, religious, sexual, political) is an important part of the vector.
a) Moving Through Autonomy Toward Interdependence
b) Developing Mature Interpersonal Relationships
c) Developing Purpose
d) Developing Integrity

7. College students ask themselves questions such as "Why do I get up in the morning?" and "Why am I here?" while moving through the vector.
a) Establishing Identity
b) Developing Mature Interpersonal Relationships
c) Developing Purpose
d) Developing Integrity

8. College students' identity development is enhanced when they have interactions with their instructors outside of class, thus are/is important at colleges and universities.
a) Teaching
b) Student Development Programs and Services
c) Friendships and Student Committees
d) Student-Faculty Relationships

9. Colleges and universities often have different goals, mission statements and school mottos, which can lead them to prioritize certain aspects of their students' identity development. Because of this, a college or a university's can significantly influence how students develop their identities at a particular school.
a) Institutional Objectives
b) Institutional Size
c) Student-Faculty Relationships
d) Friendships and Student Committees

10. College students move through the vector of college student development as they begin to question their pre-existing ideas of "right" and "wrong" and recognize that the world is not necessarily black or white.
a) Developing Competence
b) Developing Integrity
c) Developing Purpose
d) Managing Emotions 


\section{Appendix O}

Instructional Affect Assessment Instrument (McCroksey, 1994)

My attitude about this instructor is:

\begin{tabular}{|l|lllllll|r|}
\hline Good & 1 & 2 & 3 & 4 & 5 & 6 & 7 & Bad \\
Worthless & 1 & 2 & 3 & 4 & 5 & 6 & 7 & Valuable \\
Fair & 1 & 2 & 3 & 4 & 5 & 6 & 7 & Unfair \\
Positive & 1 & 2 & 3 & 4 & 5 & 6 & 7 & Negative \\
\hline
\end{tabular}

The likelihood of actually enrolling in another course with this instructor if my schedule would permit would be:

\begin{tabular}{|l|lllllll|r|}
\hline Likely & 1 & 2 & 3 & 4 & 5 & 6 & 7 & Unlikely \\
Impossible & 1 & 2 & 3 & 4 & 5 & 6 & 7 & Possible \\
Probable & 1 & 2 & 3 & 4 & 5 & 6 & 7 & Improbable \\
Would Not & 1 & 2 & 3 & 4 & 5 & 6 & 7 & Would \\
\hline
\end{tabular}




\section{Appendix $\mathrm{P}$}

Resource Management Subscale (Pintrich et al., 1991)

\begin{tabular}{|c|c|c|c|c|c|c|}
\hline $\begin{array}{c}\text { Not at all } \\
\text { true of me } \\
1\end{array}$ & 2 & 3 & 4 & 5 & 6 & $\begin{array}{c}\text { Very true } \\
\text { of me } \\
7\end{array}$ \\
\hline
\end{tabular}

1. I often feel so lazy or bored when I study for class that I quit before I finish what I planned to do.

2. I work hard to do well in class even if I don't like what we are doing.

3. When course work is difficult, I give up or only study the easy parts.

4. Even when course materials are dull and uninteresting, I manage to keep working until I finish. 


\section{Appendix Q}

Perceived Familiarity Scale (Bolkan et al., 2016)

\begin{tabular}{|c|c|c|c|c|}
\hline Not at all & Barely & A Little & Somewhat & Very Much \\
$\mathbf{1}$ & $\mathbf{2}$ & $\mathbf{3}$ & $\mathbf{4}$ & $\mathbf{5}$ \\
\hline
\end{tabular}

1. How familiar were you with the topic of this lesson before today?

2. How much did you already know about the topic of this lesson before today?

3. To what extent had you been exposed to the material in this lesson in the past? 
Appendix R

GPA Questions

1. What is your current GPA (e.g., 3.25, 4.00)?

\begin{tabular}{|c|c|c|c|c|}
\hline Not at all & Barely & A Little & Somewhat & Very \\
1 & 2 & 3 & 4 & 5 \\
\hline
\end{tabular}

2. How certain are you that the GPA you provided is accurate? 
Appendix $\mathrm{S}$

Student Interest Scale (Mazer, 2012)

\begin{tabular}{|c|c|c|c|c|}
\hline Strongly & Disagree & Neither & Agree & Strongly Agree \\
Disagree & $\mathbf{2}$ & Disagree nor & $\mathbf{4}$ & $\mathbf{5}$ \\
$\mathbf{1}$ & & Agree & & \\
& & $\mathbf{3}$ & & \\
\hline
\end{tabular}

1. I felt enthused about being in this lesson.

2. This lesson made me feel excited.

3. This lesson caused me to feel energized.

4. The topics covered in this lesson fascinated me.

5. Being in this lesson was enjoyable.

6. The lesson experience made me feel good.

7. The material fascinated me.

8. I liked the things we covered in the lesson.

9. The lesson experience felt very positive.

10. I can remember the lesson material.

11. I felt like I learned the topics covered in the lesson.

12. I could understand the flow of ideas.

13. I understood the lesson material.

14. The information covered in the lesson made me more knowledgeable.

15. The information in the lesson was useful.

16. I realized what was expected of me. 